\title{
Synthesis of Polycylic Fused Indoline Scaffolds through a Substrate-Guided Reactivity Switch
}

Cecilia Ciccolini, ${ }^{\dagger}$ Giacomo Mari, ${ }^{\dagger}$ Francesco G. Gatti, ${ }^{\ddagger}$ Giuseppe Gatti, ${ }^{\dagger}$ Gianluca Giorgi,,${ }^{\S}$ Fabio Mantellini, ${ }^{\dagger}$ and Gianfranco Favi ${ }^{*}, \dagger$

'Department of Biomolecular Sciences, Section of Chemistry and Pharmaceutical Technologies, University of Urbino "Carlo Bo”, Via I Maggetti 24, 61029 Urbino, Italy

"Department of Chemistry, Materials and Chemical Engineering “G. Natta”, Piazza Leonardo da Vinci 32, 20133 Milano, Italy

§Department of Biotechnologies, Chemistry \& Pharmacy, University of Siena, Via A. Moro 2, 53100 Siena, Italy

e-mail: gianfranco.favi@uniurb.it

\section{SUPPORTING INFORMATION}

Table of Contents

1. $\quad$ Starting materials (Figure S1) S2

2. $\quad$ Optimization for reaction of $\mathbf{1 a}$ with cyclic azoalkene 2a (Table S1) S3

3. X-ray structure of compound 3e (CCDC: 1950877) S4

4. $\quad{ }^{1} \mathrm{H}$ and ${ }^{13} \mathrm{C}$ NMR spectra of all products $\quad$ S5-S69 


\section{Starting materials (Figure S1):}

\section{Indoles}<smiles>Cn1ccc2ccccc21</smiles>

1a<smiles>Cn1ccc2ccc(Cl)cc21</smiles>

1b<smiles>CC(=O)c1ccc2c(ccn2C)c1</smiles>

1c<smiles>Cn1ccc2cc(C#N)ccc21</smiles><smiles>Cn1ccc2cc(C=O)ccc21</smiles>

1e<smiles>Cn1ccc2cc([N+](=O)[O-])ccc21</smiles><smiles>COc1ccc2c(ccn2C)c1</smiles>

$1 \mathrm{~g}$<smiles>Cc1cccc2ccn(C)c12</smiles><smiles>CCn1ccc2ccccc21</smiles><smiles>CCCn1ccc2cc(C)ccc21</smiles><smiles>c1ccc(Cn2ccc3ccccc32)cc1</smiles><smiles>c1ccc2[nH]ccc2c1</smiles><smiles>Cc1ccc2[nH]ccc2c1</smiles><smiles>Cn1ccc2cccnc21</smiles>

1n<smiles>c1ccc2c3c([nH]c2c1)CCC3</smiles><smiles>c1ccc2c3c([nH]c2c1)CCCC3</smiles>

$1 t$<smiles>Cc1[nH]c2ccc3ccccc3c2c1C</smiles>

1y<smiles>Cc1cc2ccccc2n1C</smiles>

10

$\mathrm{Cl}$<smiles>CCc1c(C)[nH]c2ccc(I)cc12</smiles><smiles>Cc1c(C)n(C)c2ccccc12</smiles>

$1 z$<smiles>Cc1c[nH]c2ccccc12</smiles>

$1 p$<smiles>CCc1c(C)[nH]c2ccc(OC)cc12</smiles>

19<smiles>COc1ccc2[nH]c3c(c2c1)CCCCC3</smiles>

Azoalkenes<smiles>CCOC(=O)C1=C(N=NC(N)=O)CCCC1</smiles>

2a

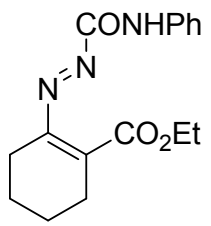

2b

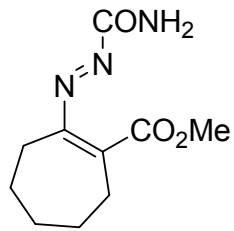

2c

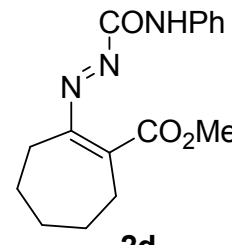

2d

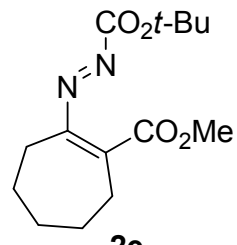

$2 \mathrm{e}$

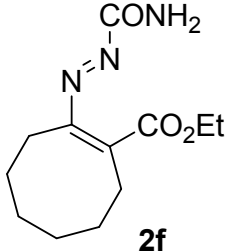

$2 f$<smiles>CCOC(=O)C1=C(N=NC(=O)OC(C)(C)C)CCCCCC1</smiles><smiles>CCOC(=O)C1=C(N=NC(N)=O)CCC1</smiles><smiles>[O-]N=NC1=CCCCC1</smiles><smiles>COC(=O)C=C(C)NNC(C)=O</smiles><smiles>CCOC=C(C)NNC(=O)OCC</smiles><smiles>CC(=O)OC=C(C)N=NC(=O)OC(C)(C)C</smiles><smiles>C/C(=C/NC(=O)OCc1ccccc1)C(=O)OC(C)(C)C</smiles>

$2 \mathrm{i}$

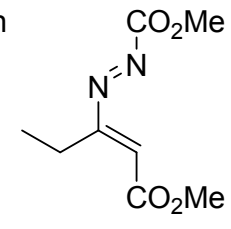

$2 p$<smiles>CCC/C(=C/C(C)=O)N=NC(C)=O</smiles>

2q

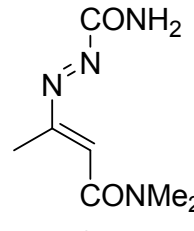

$2 r$

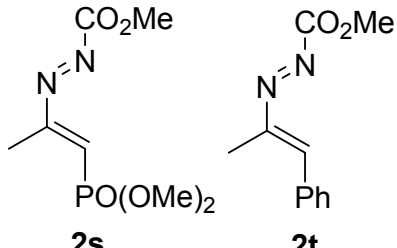

2s 
2. Supplementary Table (Table S1). Optimization Conditions for Reaction of $\mathrm{N}$ Methylindole (1a) with Cyclic Azoalkene 2a. ${ }^{a}$
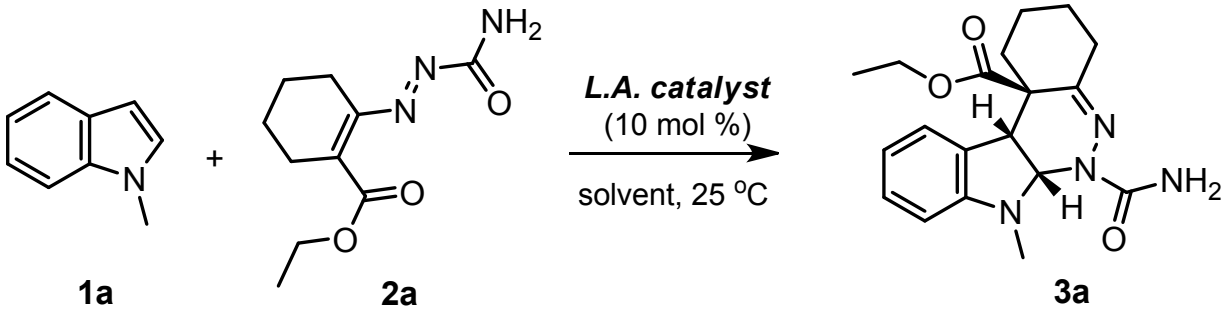

\begin{tabular}{|cccc|}
\hline entry & catalyst & solvent & yield (\%) $)^{\boldsymbol{b}}$ \\
1 & - & $\mathrm{CH}_{2} \mathrm{Cl}_{2}$ & 0 \\
2 & $\mathrm{Sc}(\mathrm{OTf})_{3}$ & $\mathrm{CH}_{2} \mathrm{Cl}_{2}$ & 0 \\
3 & $\mathrm{Zn}(\mathrm{OAc})_{2}$ & $\mathrm{CH}_{2} \mathrm{Cl}_{2}$ & 0 \\
4 & $\mathrm{ZnSO}_{4}$ & $\mathrm{CH}_{2} \mathrm{Cl}_{2}$ & 0 \\
5 & $\mathrm{Zn}(\mathrm{OTf})_{2}$ & $\mathrm{CH}_{2} \mathrm{Cl}_{2}$ & 0 \\
6 & $\mathrm{SnCl}_{3} 6 \mathrm{H}_{2} \mathrm{O}$ & $\mathrm{CH}_{2} \mathrm{Cl}_{2}$ & $<5$ \\
7 & $\mathrm{LiClO}_{4}$ & $\mathrm{CH}_{2} \mathrm{Cl}_{2}$ & 0 \\
8 & $\mathrm{LiCl}_{2}$ & $\mathrm{CH}_{2} \mathrm{Cl}_{2}$ & 0 \\
9 & $\mathrm{CuCl}_{2}$ & $\mathrm{CH}_{2} \mathrm{Cl}_{2}$ & 0 \\
10 & ${\mathrm{Cu}(\mathrm{OTf})_{2}}_{2}$ & $\mathrm{CH}_{2} \mathrm{Cl}_{2}$ & $<5$ \\
11 & $\mathrm{CuBr}_{2}$ & $\mathrm{CH}_{2} \mathrm{Cl}_{2}$ & 0 \\
12 & $\mathrm{InBr}_{3}$ & $\mathrm{CH}_{2} \mathrm{Cl}_{2}$ & 0 \\
13 & $\mathrm{ZnBr}_{2}$ & $\mathrm{CH}_{2} \mathrm{Cl}_{2}$ & 37 \\
14 & $\mathrm{ZnCl}_{2}$ & $\mathrm{CH}_{2} \mathrm{Cl}_{2}$ & 39 \\
\hline $15^{c}$ & $\mathrm{ZnCl}_{2}$ & $\mathrm{CH}_{2} \mathrm{Cl}_{2}$ & 50 \\
\hline $16^{c}$ & $\mathrm{ZnCl}_{2}$ & $\mathrm{Acetone}^{c}$ & 43 \\
$17^{c}$ & $\mathrm{ZnCl}_{2}$ & $\mathrm{THF}_{2}$ & 40 \\
$18^{c}$ & $\mathrm{ZnCl}_{2}$ & $\mathrm{CH}_{3} \mathrm{CN}_{2}$ & 23 \\
$19^{c}$ & $\mathrm{ZnCl}_{2}$ & $\mathrm{Cyclohexane}^{c}$ & $<5$ \\
\hline
\end{tabular}

${ }^{a}$ Reaction conditions: 1a $(0.3 \mathrm{mmol}), \mathbf{2 a}(0.3 \mathrm{mmol})$, cat. $(0.03 \mathrm{mmol}$, $10 \mathrm{~mol} \%)$, DCM $(2.0 \mathrm{~mL}), 25{ }^{\circ} \mathrm{C}$ for $12 \mathrm{~h} .{ }^{b}$ Yields determined by ${ }^{1} \mathrm{H}$ NMR analysis of the crude mixture using 1,1,2,2 tetrachloroethane as the internal standard. ${ }^{c} 2.0$ equiv of 1 a was used.

Our work commenced by studying the reaction between indole 1a and cyclic 1,2-diaza-1,3diene 2a (Table S1). No reaction took place, and both compounds remained inactive in the absence of Lewis acid catalyst. A series of Lewis acid catalysts [such as $\operatorname{Sc}(\mathrm{OTf})_{3}$, $\mathrm{Zn}(\mathrm{OAc})_{2}, \mathrm{ZnSO}_{4}, \mathrm{Zn}(\mathrm{OTf})_{2}, \mathrm{SmCl}_{3} \cdot 6 \mathrm{H}_{2} \mathrm{O}, \mathrm{LiClO}_{4}, \mathrm{LiCl}, \mathrm{CuCl}_{2}, \mathrm{Cu}(\mathrm{OTf})_{2}, \mathrm{CuBr}_{2}, \mathrm{InBr}_{3}$, 
$\mathrm{ZnBr}_{2}$, and $\mathrm{ZnCl}_{2}$ ] and solvents [such as dichloromethane, acetone, tetrahydrofuran, acetonitrile and cyclohexane] were examined, and the combination of $\mathrm{ZnCl}_{2}$ and $\mathrm{CH}_{2} \mathrm{Cl}_{2}$ (heterogeneous catalytic system) was found to be superior for this transformation. Noteworthy, compound 3a was obtained as a single regio- and diastereoisomer (50\% yield).

The relative stereochemistry of cycloadducts $\mathbf{3}$ were assigned based on the X-ray diffraction analysis of compound $\mathbf{3 e}$.

\section{X-ray structure of compound 3e (CCDC: 1950877)}

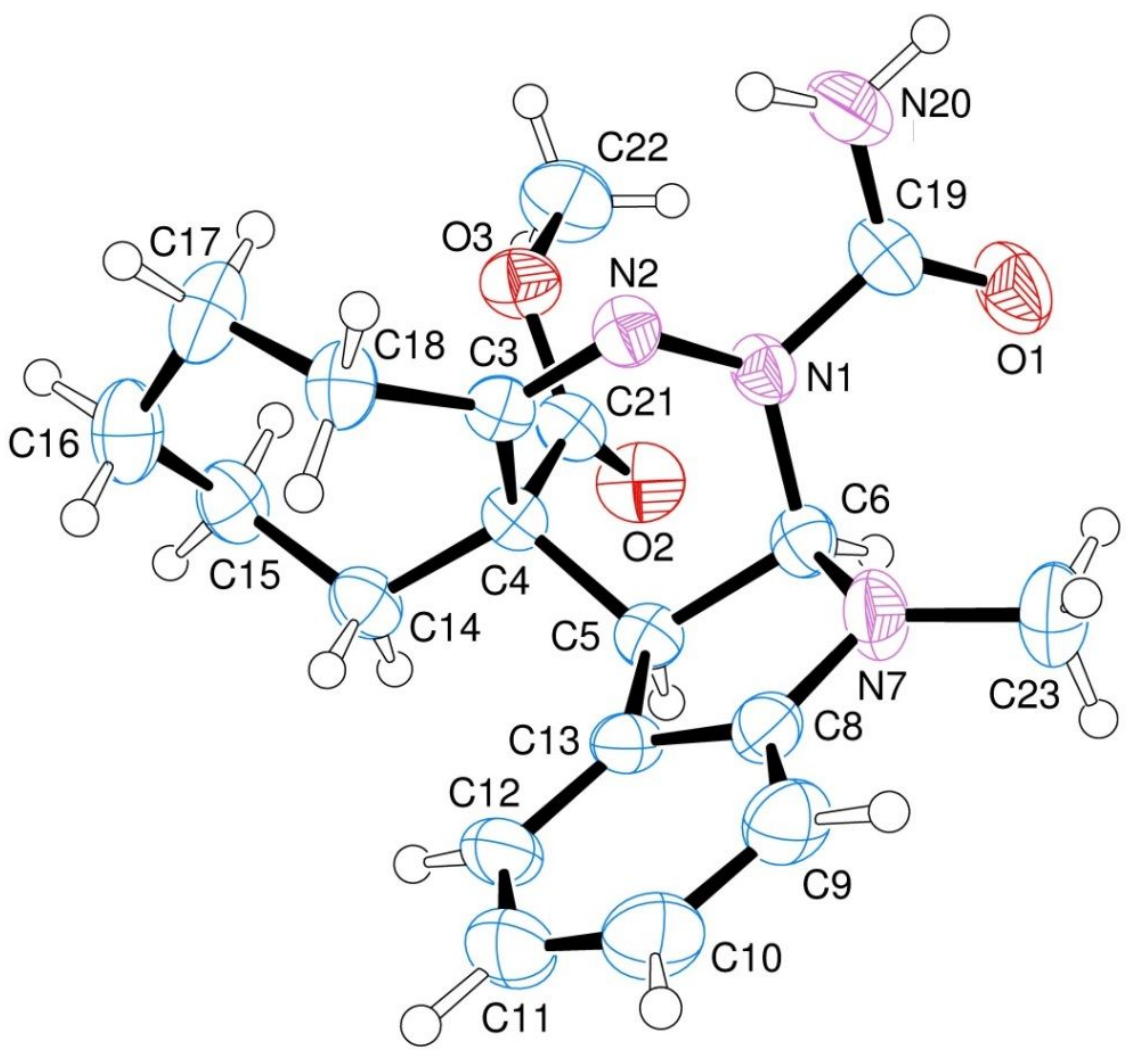

Ellipsoids enclose 50\% probability 


\section{4. ${ }^{1} \mathrm{H}$ and ${ }^{13} \mathrm{C}$ NMR spectra of products.}

${ }^{1} \mathrm{H}$ NMR of 3 a (400 MHz, DMSO- $\left.d_{6}\right)$<smiles>CCOC[C@]12CCCCC1=NN(C(N)=O)[C@H]1N(C)c3ccccc3[C@@]12C</smiles>

3a<smiles>c1ccccc1</smiles>

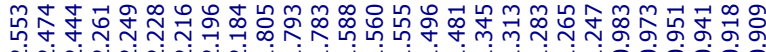

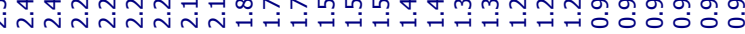

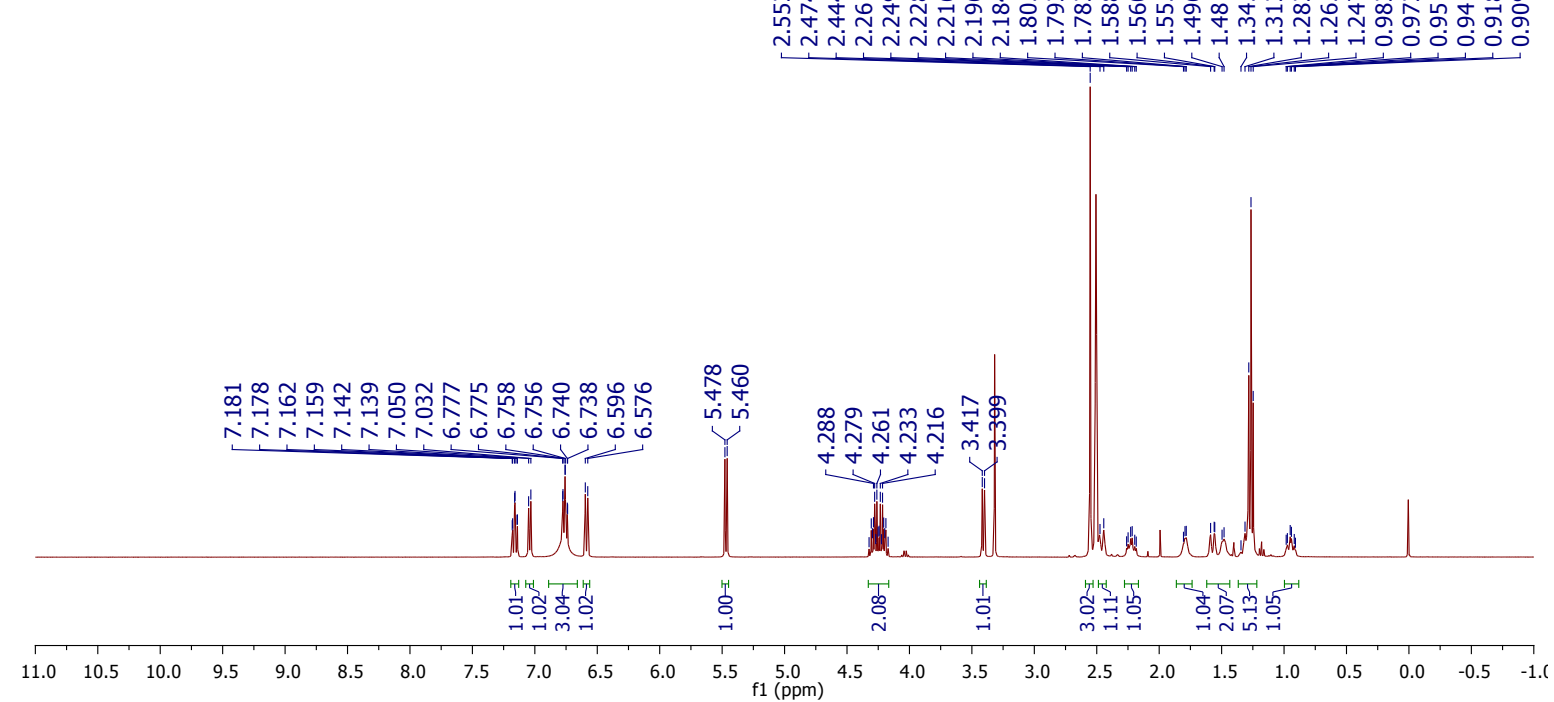

${ }^{13} \mathrm{C}$ NMR of 3a (100 MHz, $\left.\mathrm{CDCl}_{3}\right)$

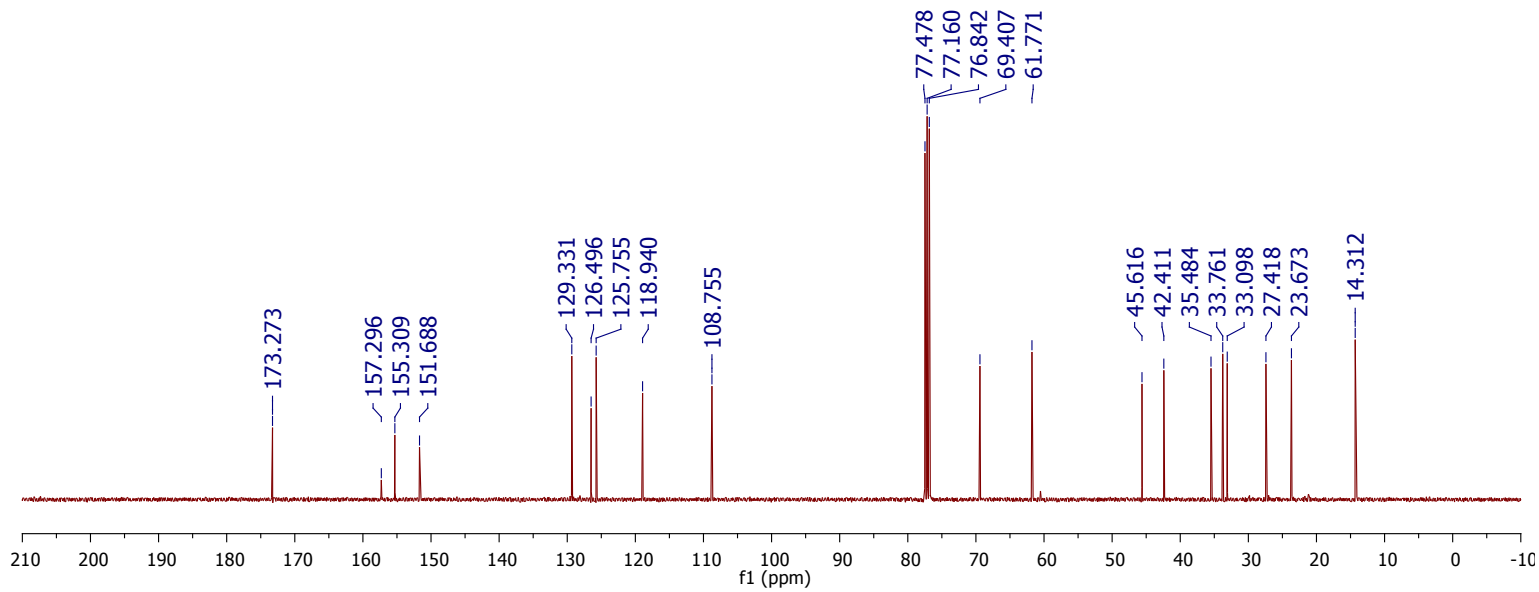


${ }^{1} \mathrm{H}-{ }^{1} \mathrm{H}$ COSY NMR of 3a (400 MHz, DMSO- $\left.d_{6}\right)$

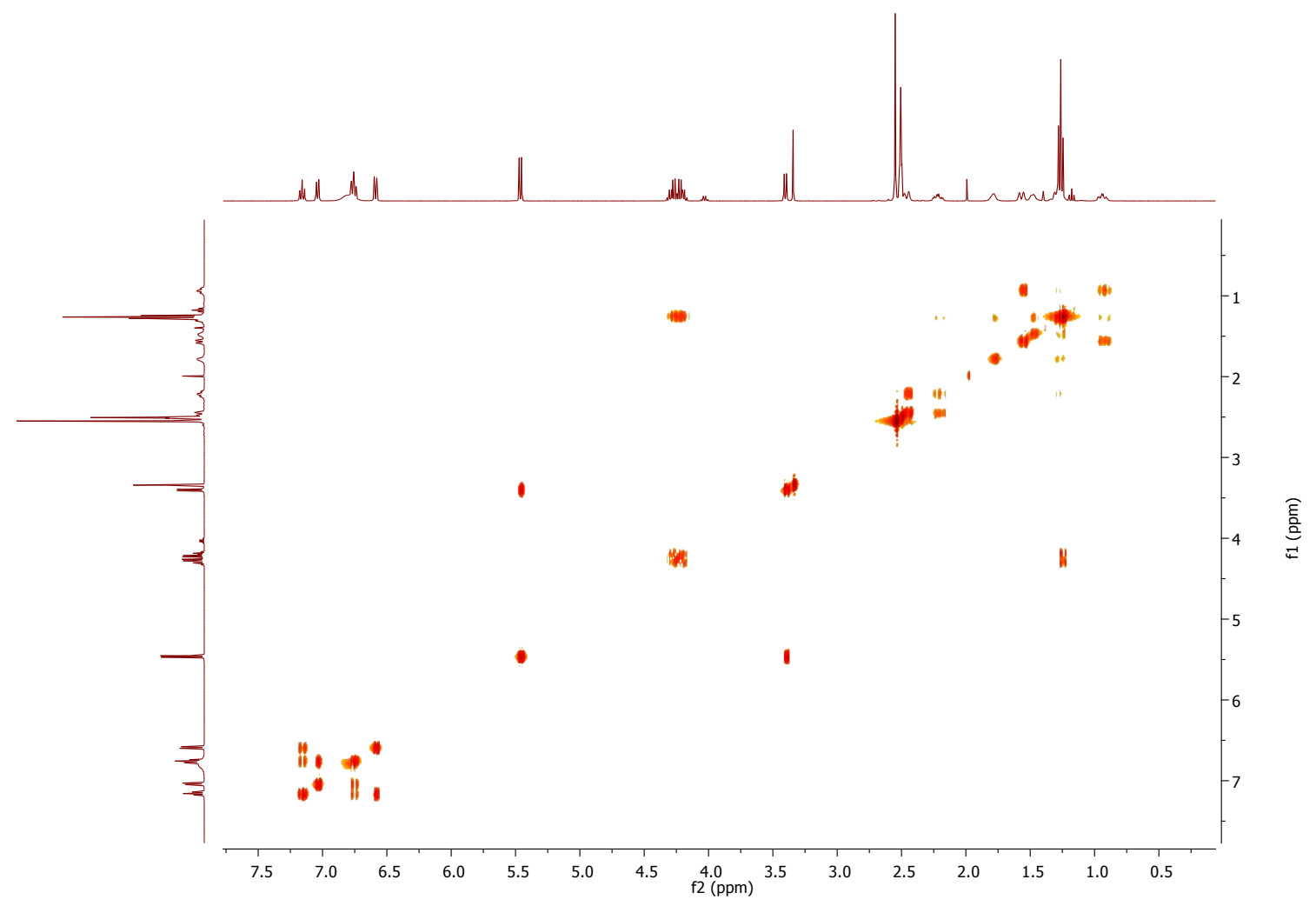

HMQC NMR of 3a (400 MHz, DMSO- $\left.d_{6}\right)$

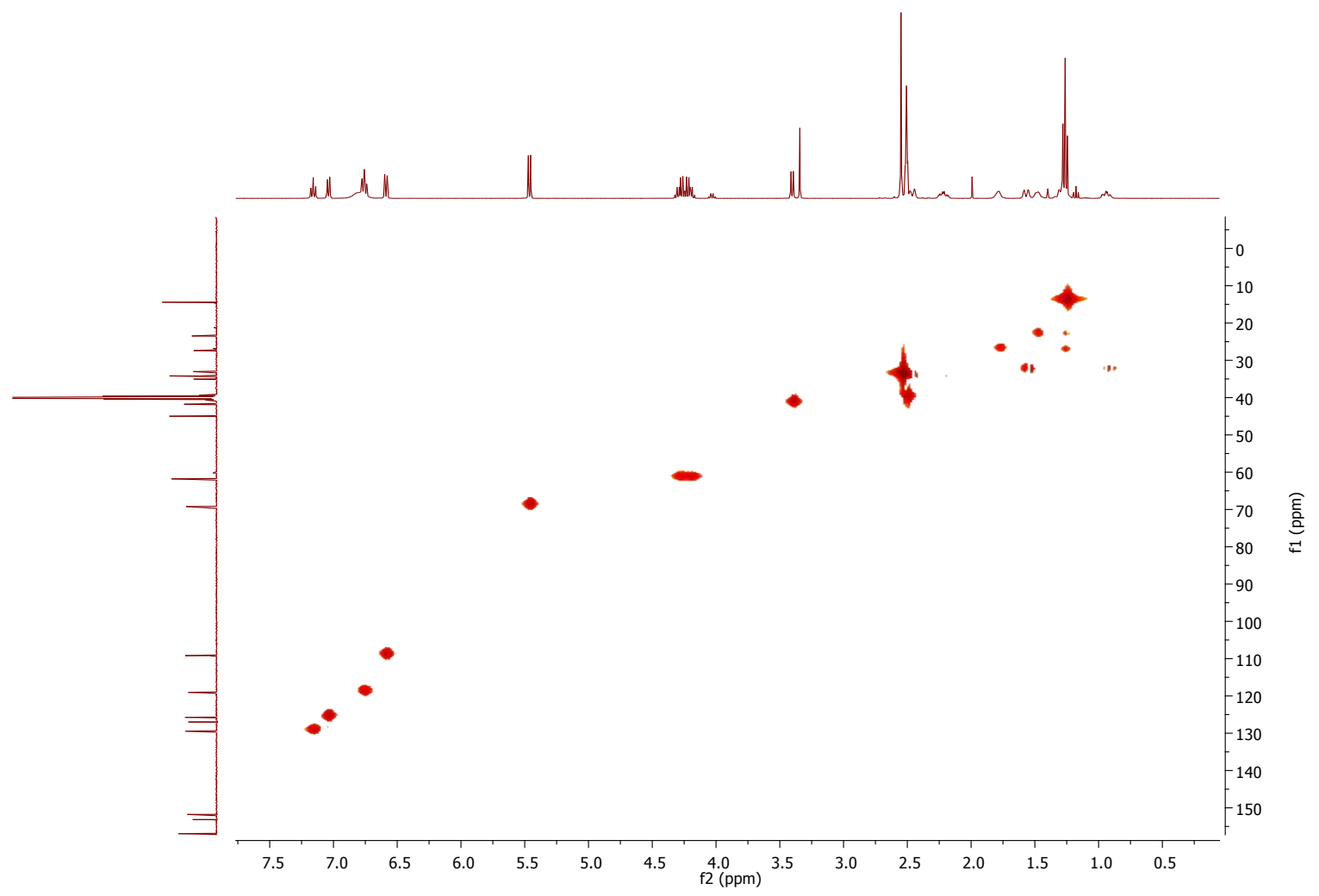


HMBC NMR of 3a (400 MHz, DMSO- $d_{6}$ )

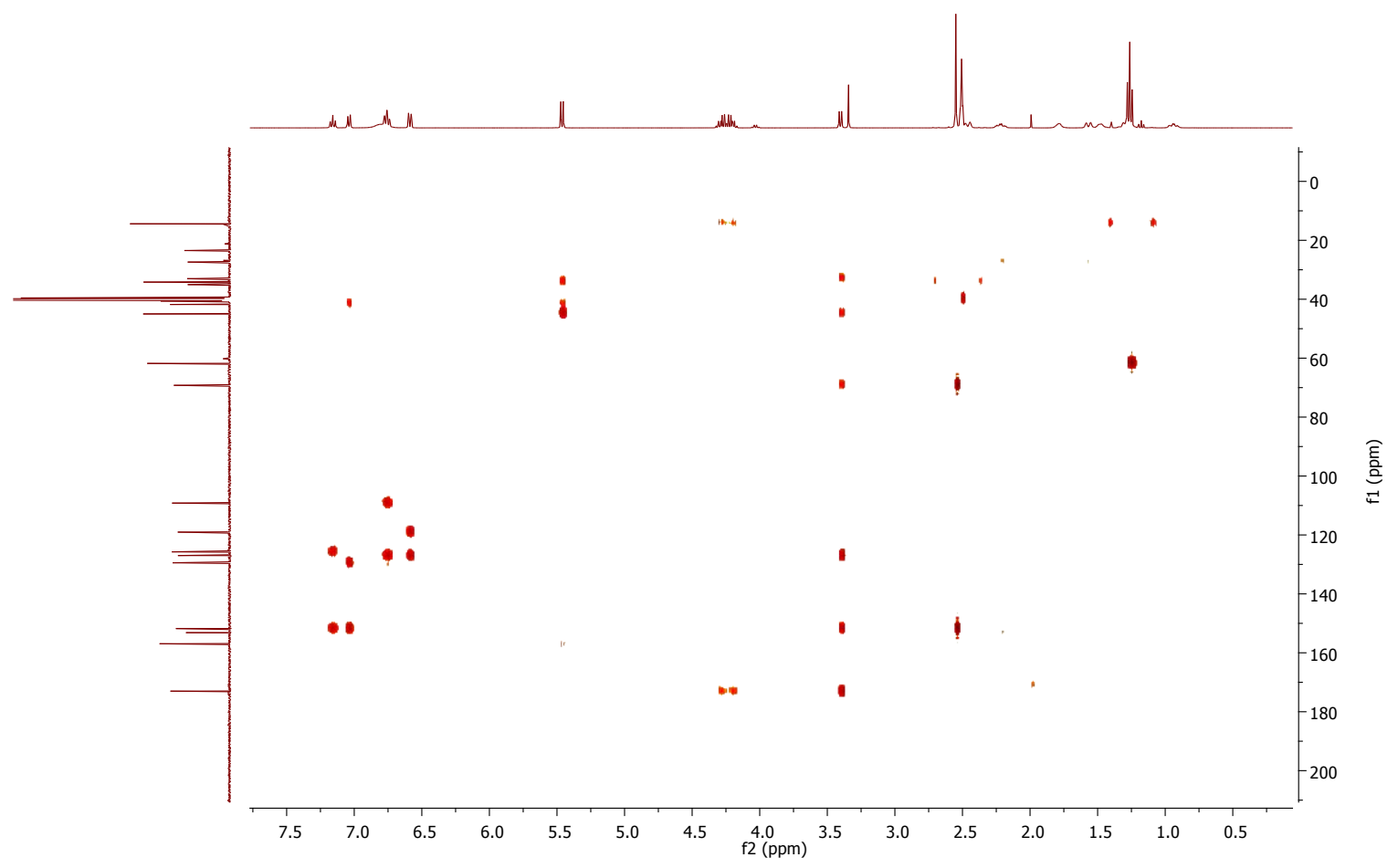


${ }^{1} \mathrm{H}$ NMR of $3 \mathrm{~b}$ (400 MHz, DMSO- $\left.d_{6}\right)$
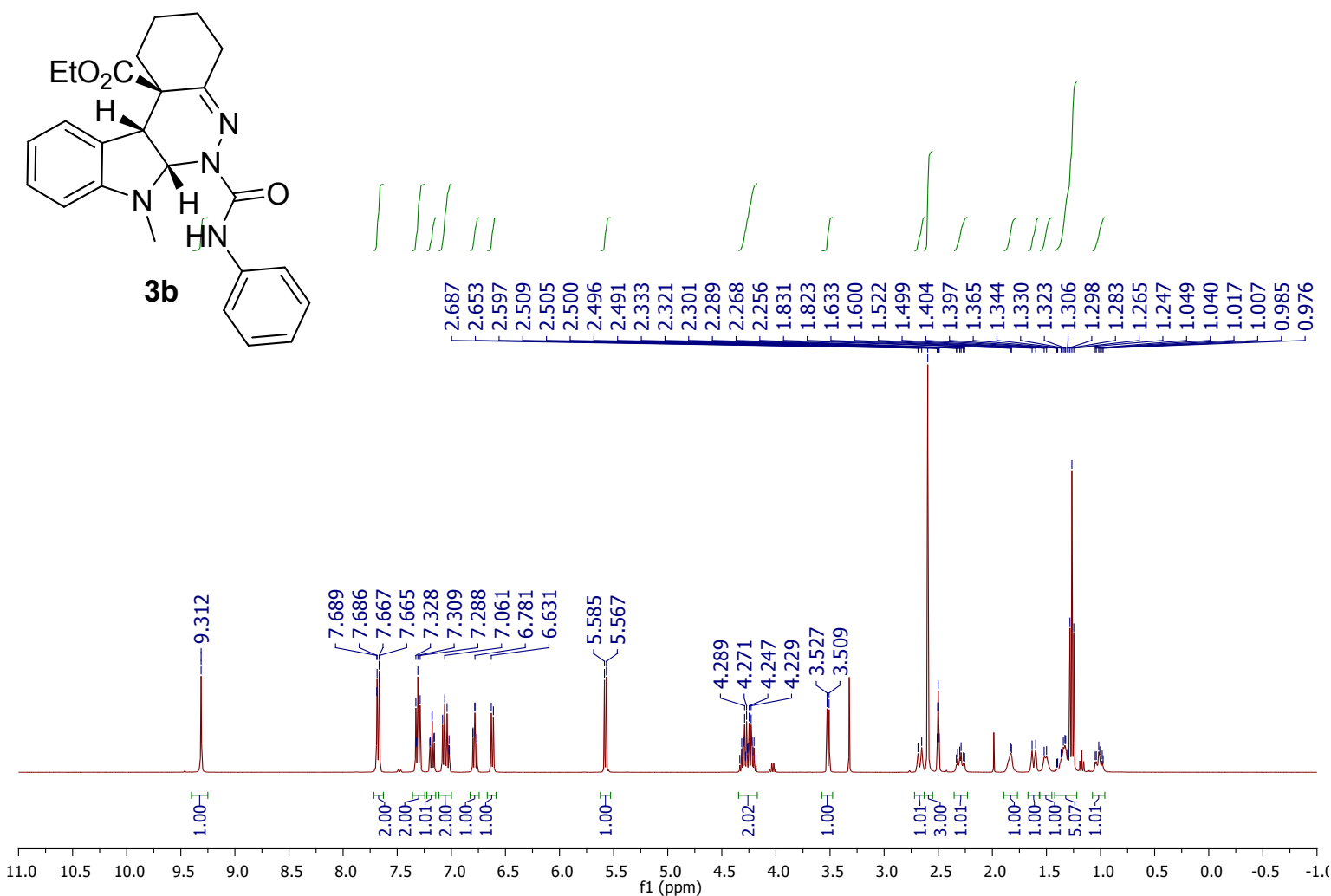

${ }^{13}$ C NMR of 3b (100 MHz, DMSO- $\left.d_{6}\right)$

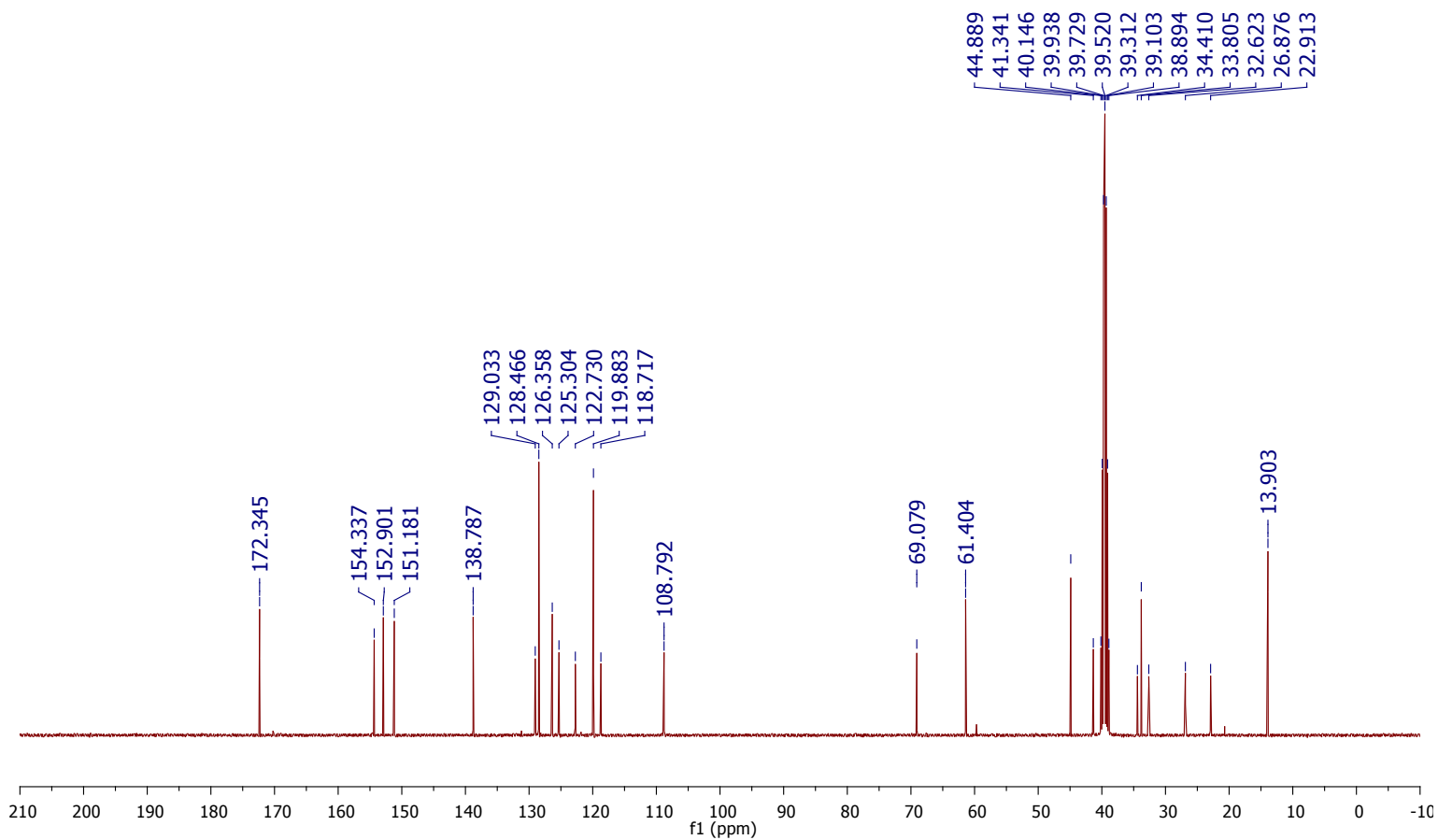


${ }^{1}$ H NMR of 3c (400 MHz, DMSO- $\left.d_{6}\right)$

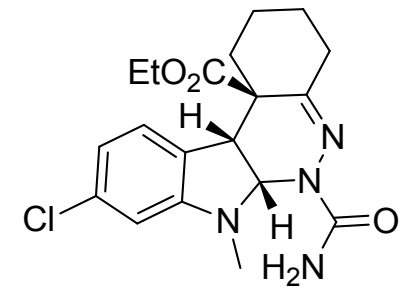

$3 c$<smiles>Cc1ccccc1</smiles>

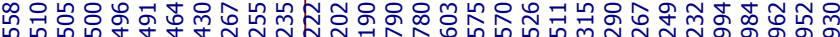
ำn

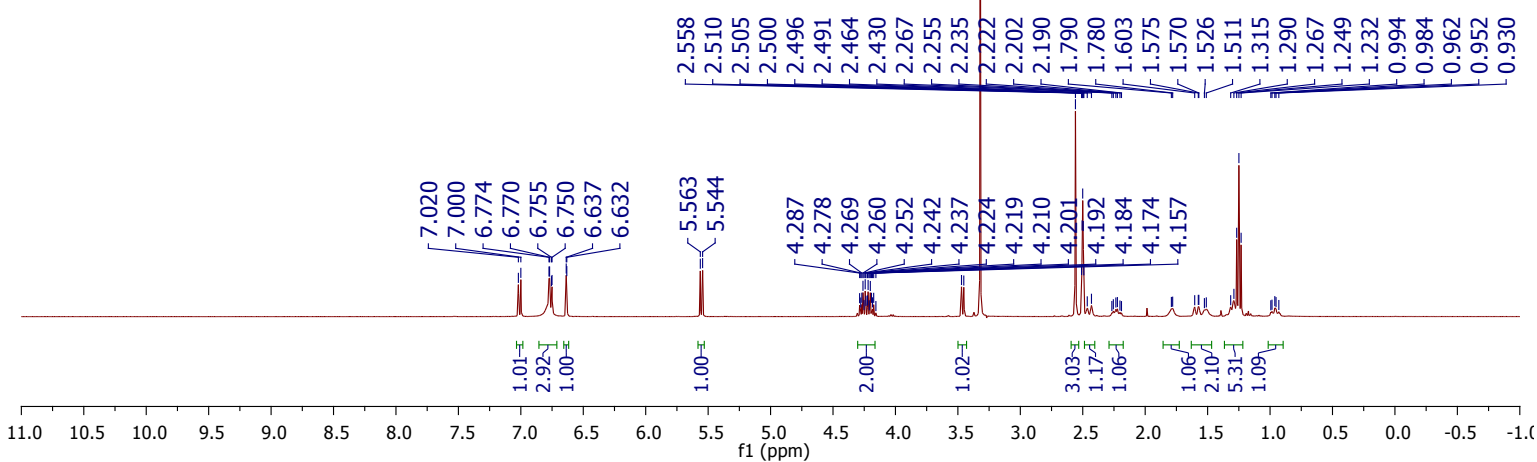

${ }^{13}$ C NMR of 3c (100 MHz, DMSO-d 6$)$

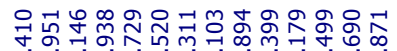
就

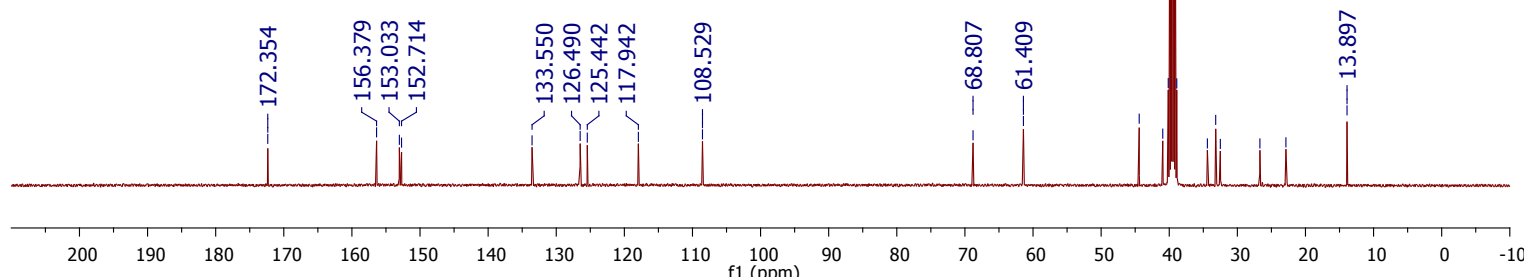


${ }^{1} \mathrm{H}$ NMR of 3d (400 MHz, DMSO- $\left.d_{6}\right)$
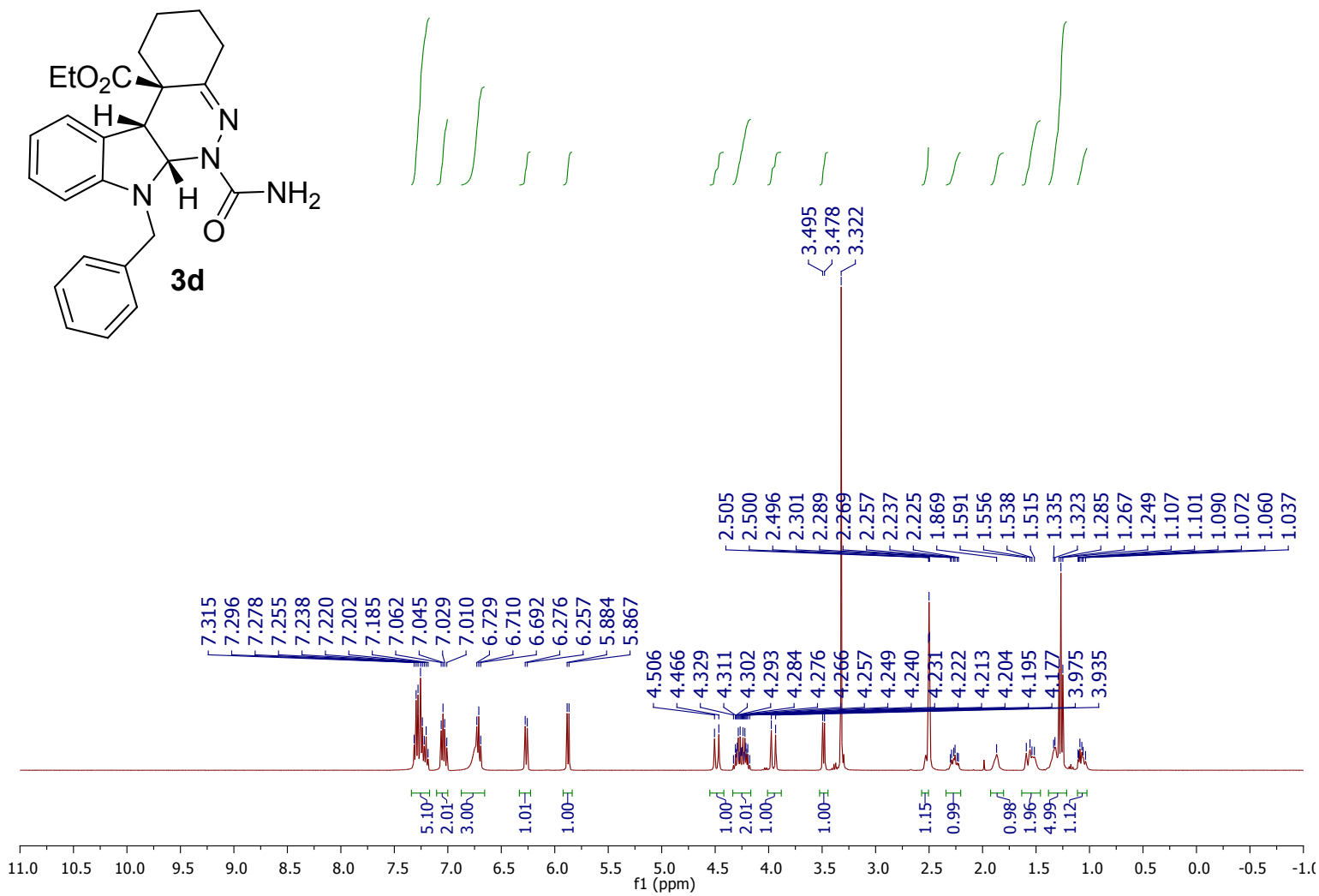

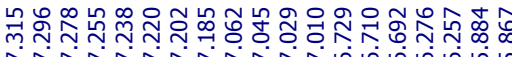

${ }^{13} \mathrm{C}$ NMR of $3 \mathrm{~d}$ (100 MHz, DMSO- $\left.d_{6}\right)$

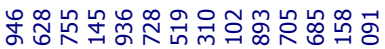

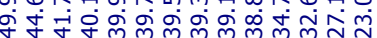

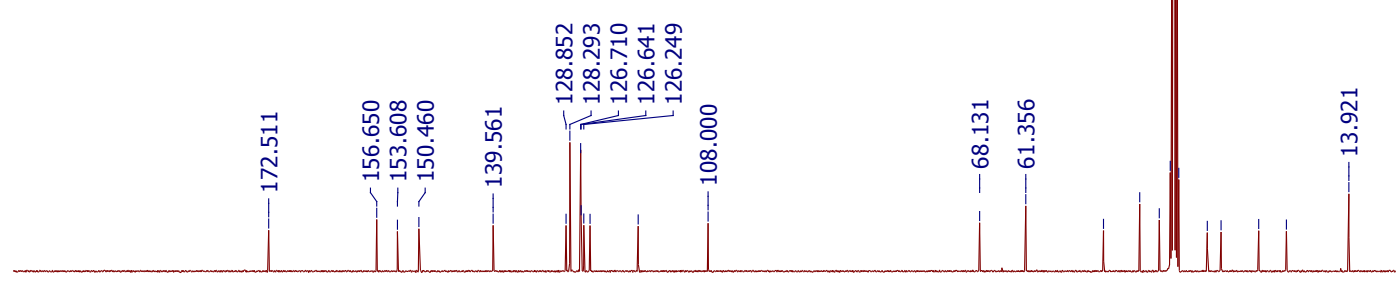

$\begin{array}{lllllllllllllllllllllllllllllll}210 & 200 & 190 & 180 & 170 & 160 & 150 & 140 & 130 & 120 & 110 & 100 & 90 & 80 & 70 & 60 & 50 & 40 & 30 & 20 & 10 & 0 & -10\end{array}$ 
NOESY NMR of 3d (400 MHz, DMSO- $\left.d_{6}\right)$

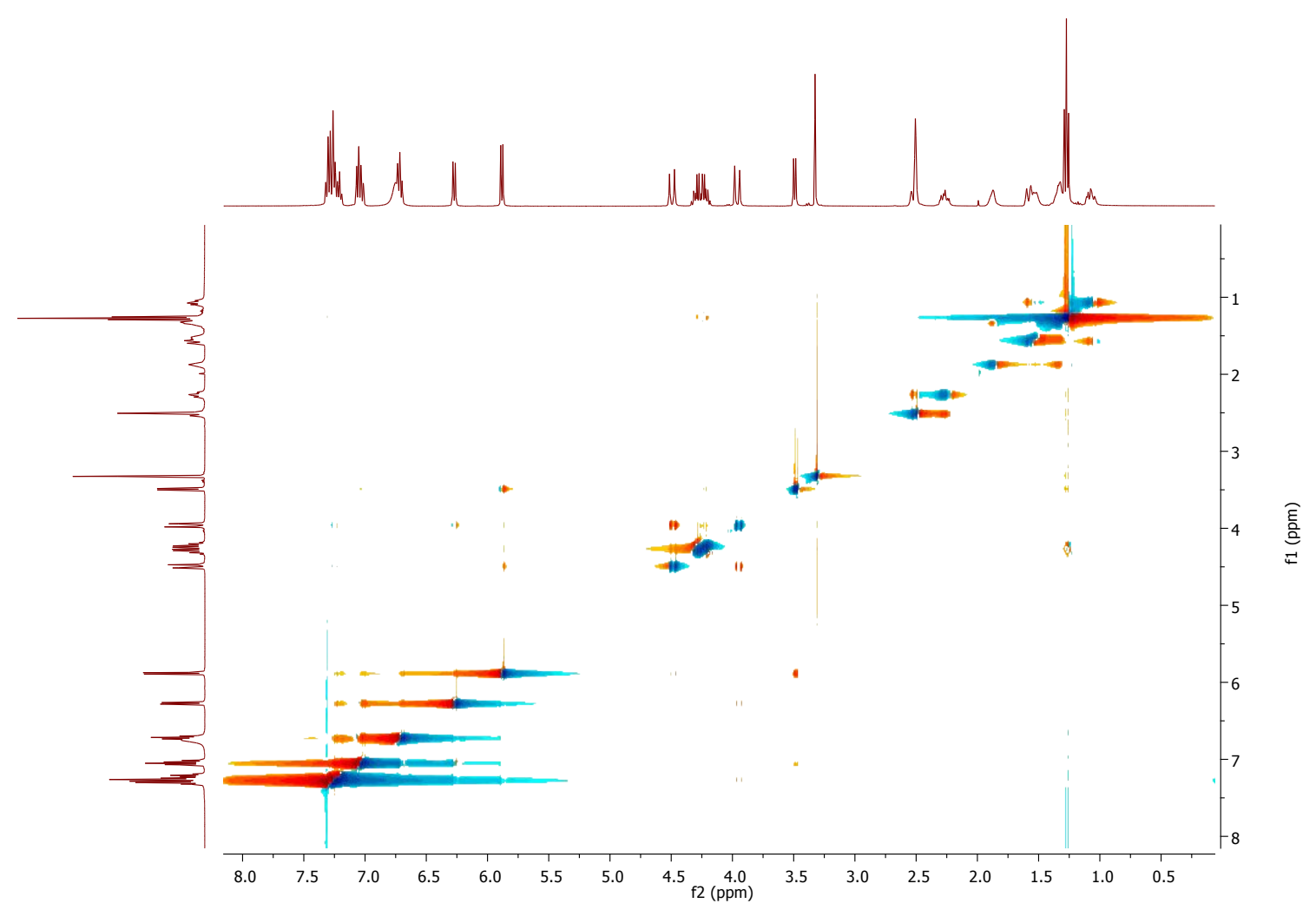


${ }^{1} \mathrm{H}$ NMR of 3e (400 MHz, DMSO- $\left.d_{6}\right)$
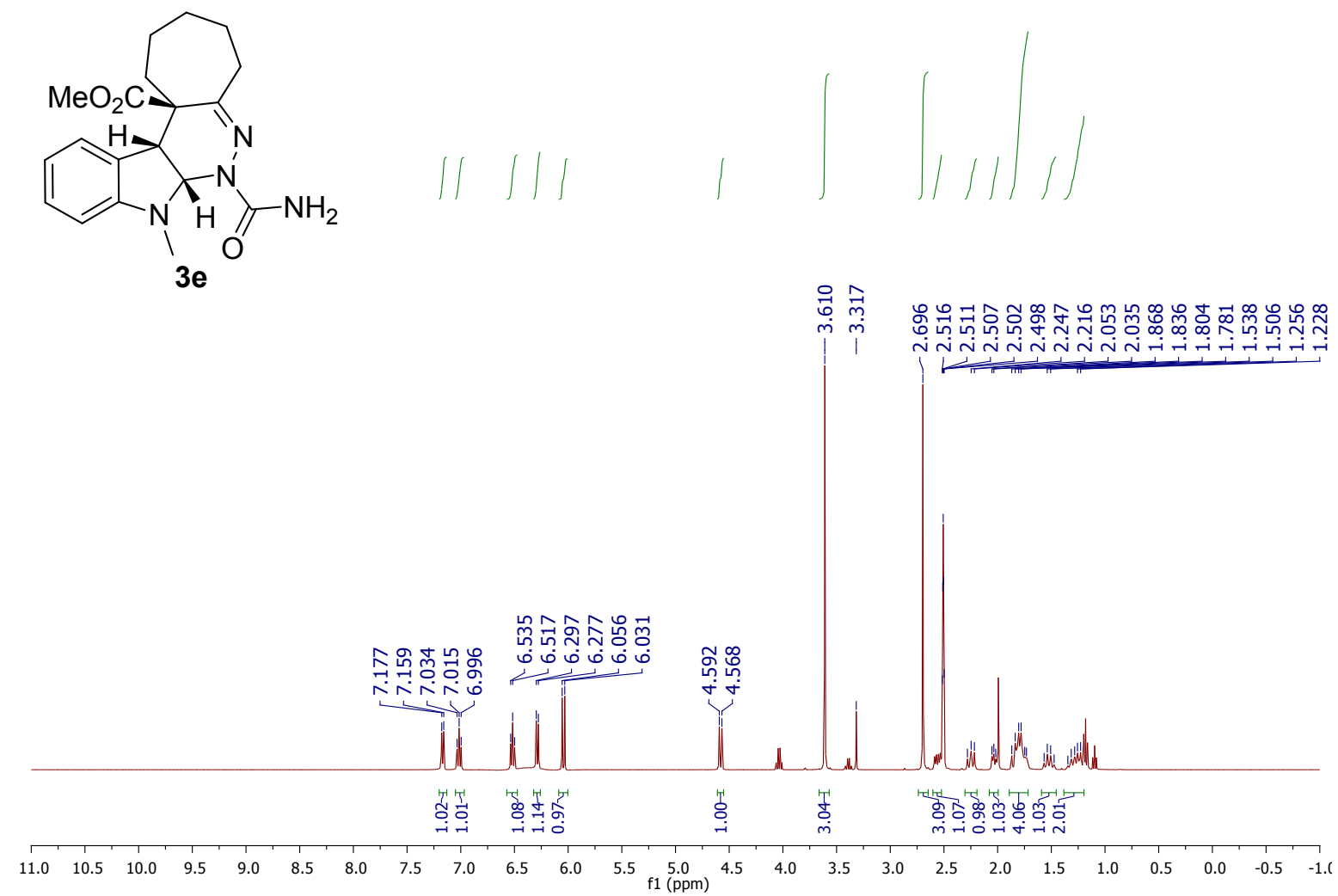

${ }^{13} \mathrm{C}$ NMR of $3 \mathrm{e}$ (100 MHz, DMSO- $\left.d_{6}\right)$

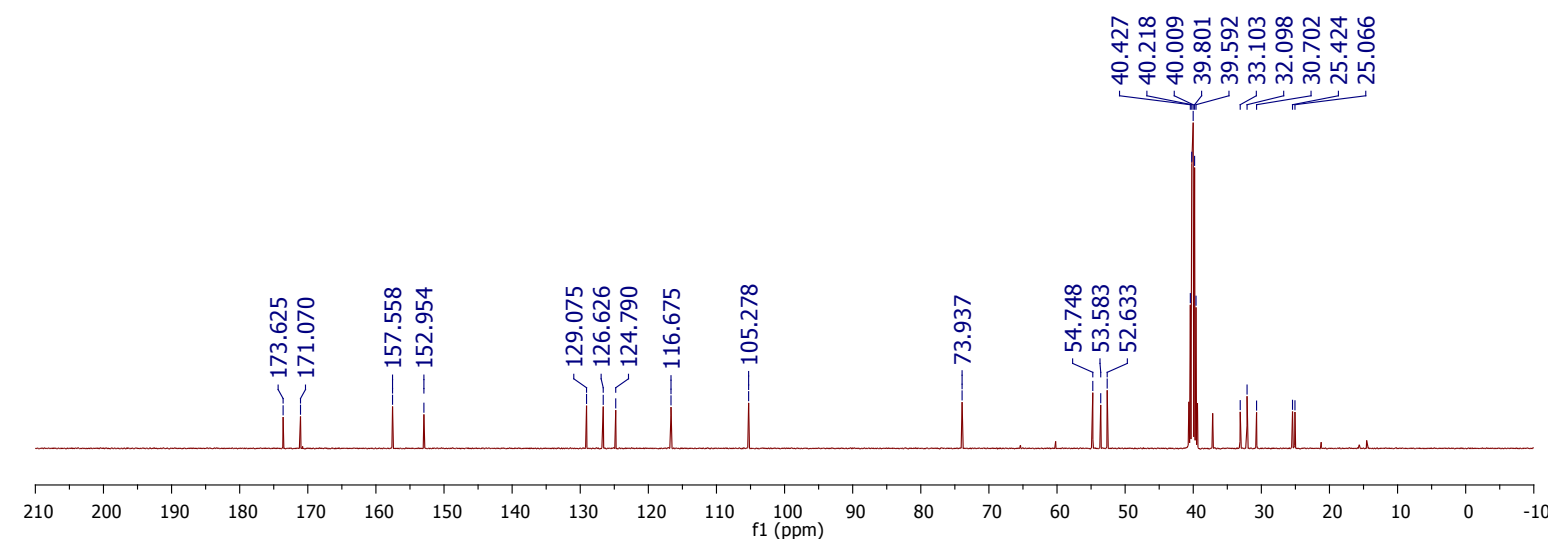


${ }^{1} \mathrm{H}$ NMR of $3 \mathrm{f}$ (400 MHz, DMSO- $\left.d_{6}\right)$

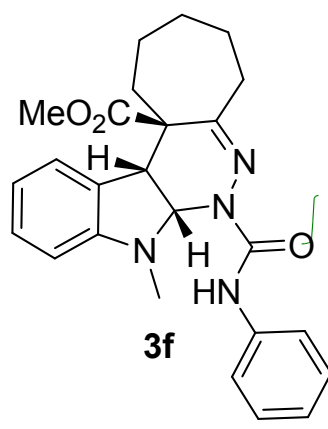
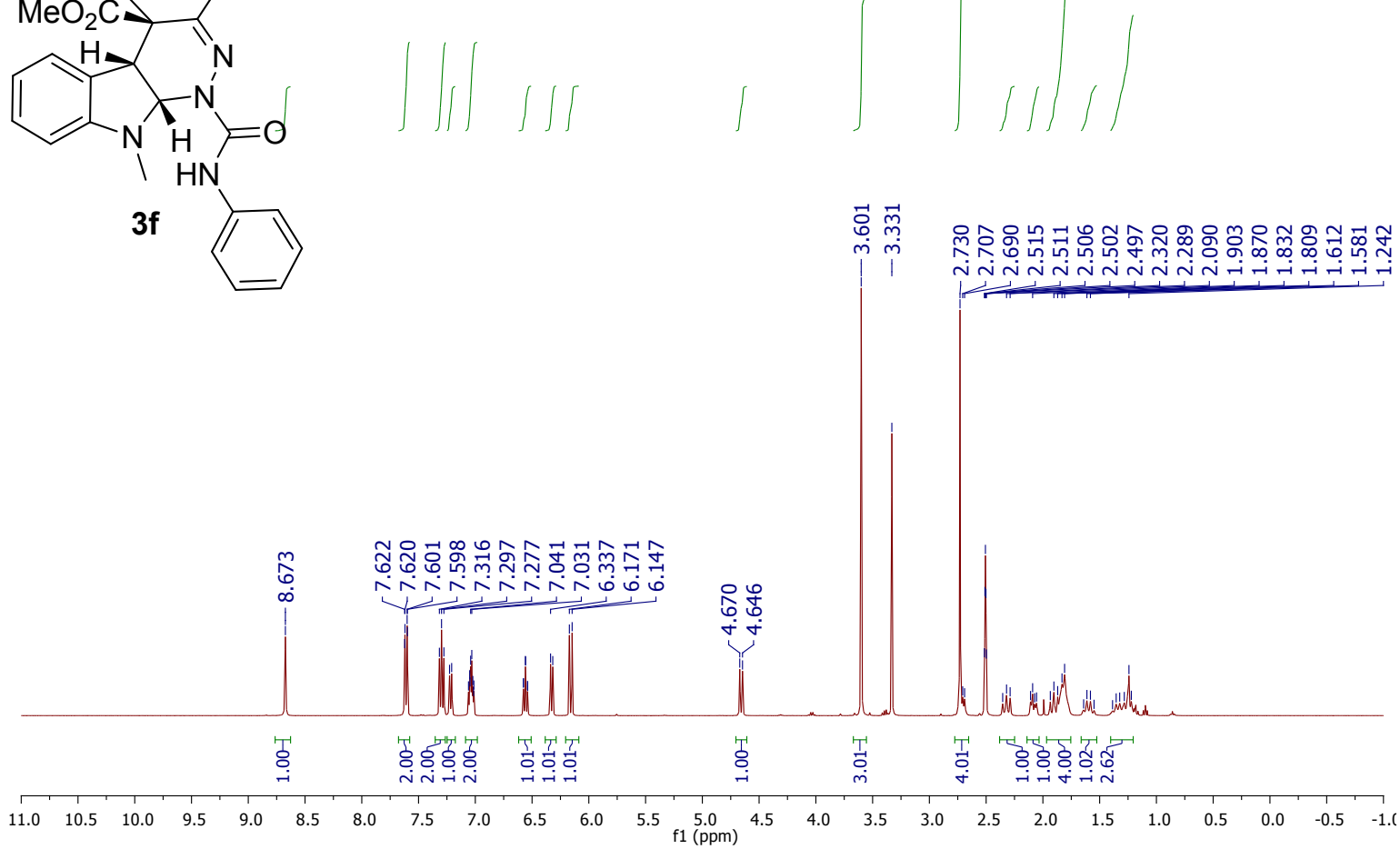

${ }^{13} \mathrm{C}$ NMR of $3 \mathrm{f}\left(100 \mathrm{MHz}, \mathrm{DMSO}-d_{6}\right)$

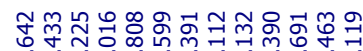

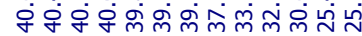

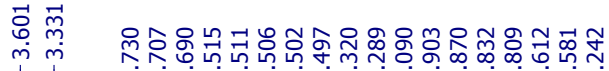
iñ

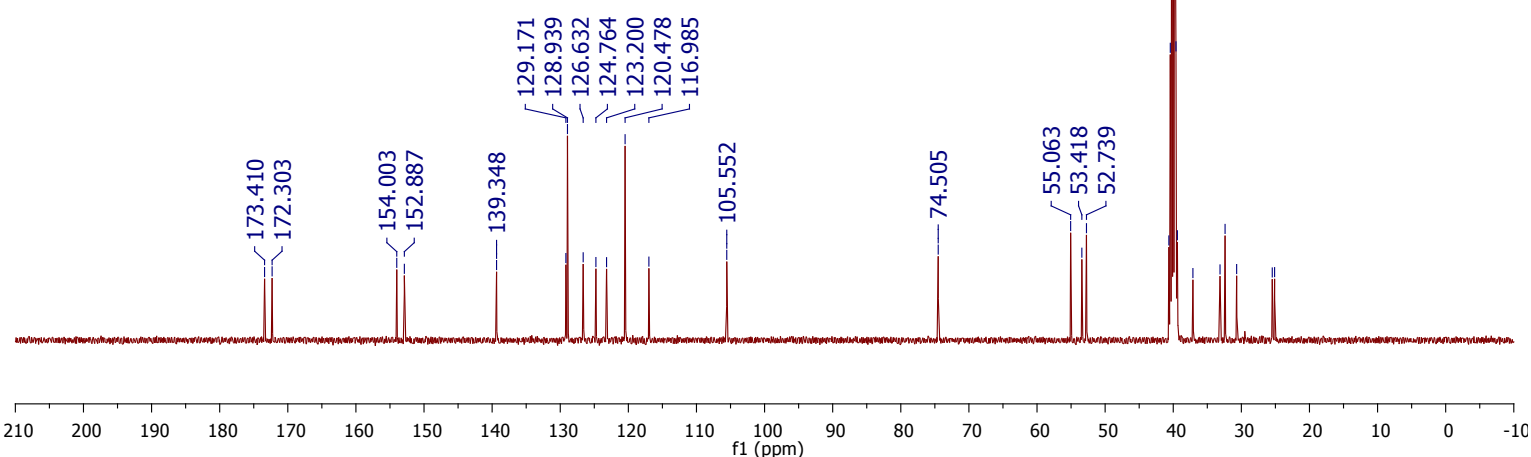


${ }^{1} \mathrm{H}$ NMR of $3 \mathrm{~g}$ (400 MHz, DMSO- $\left.d_{6}\right)$
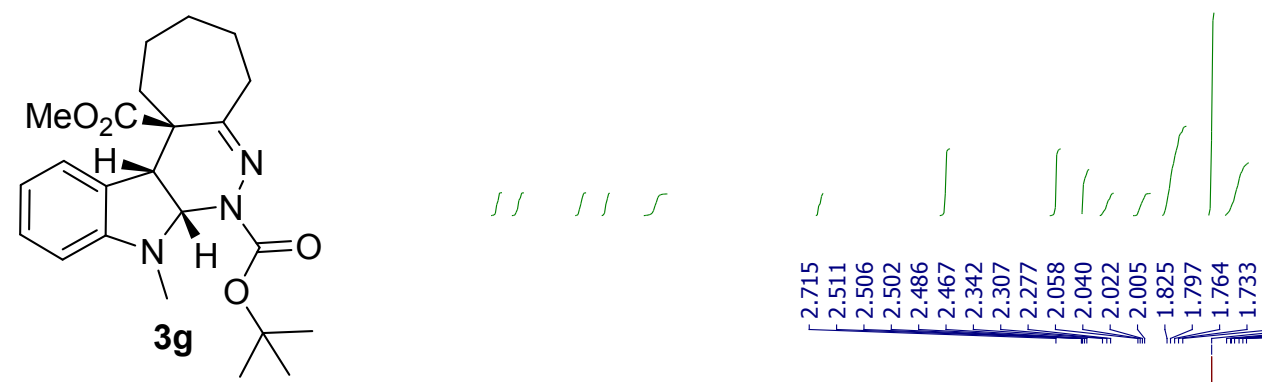

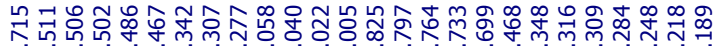

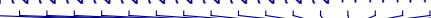

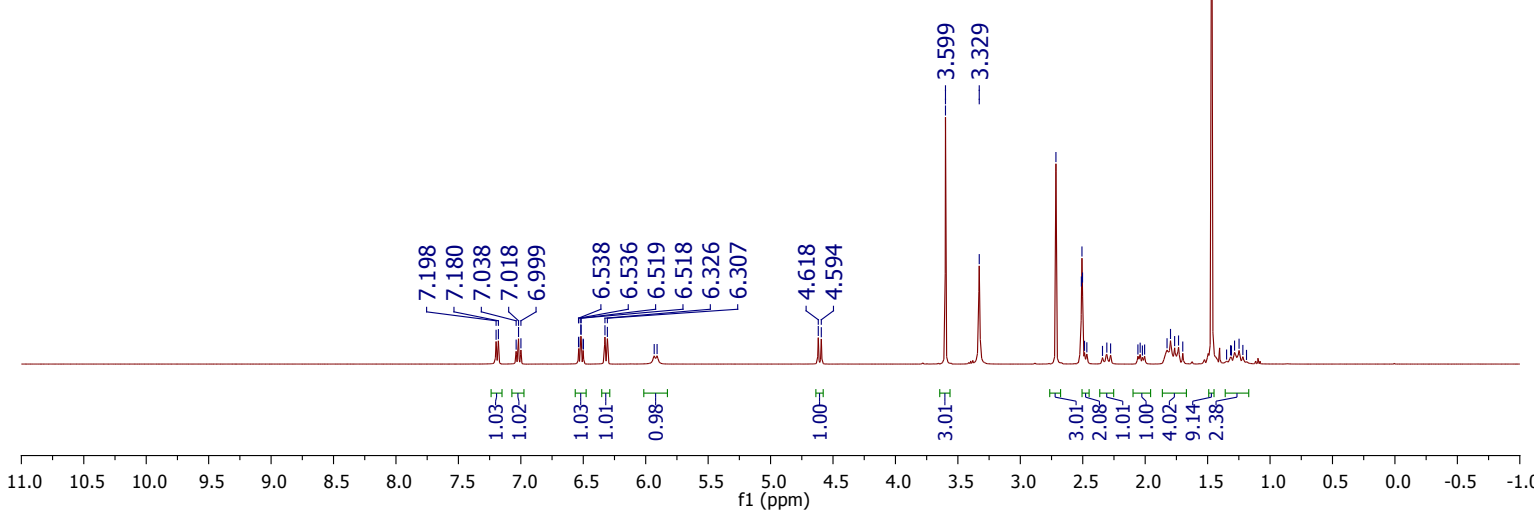

${ }^{13} \mathrm{C}$ NMR of $3 \mathrm{~g}$ (100 MHz, DMSO- $\left.d_{6}\right)$

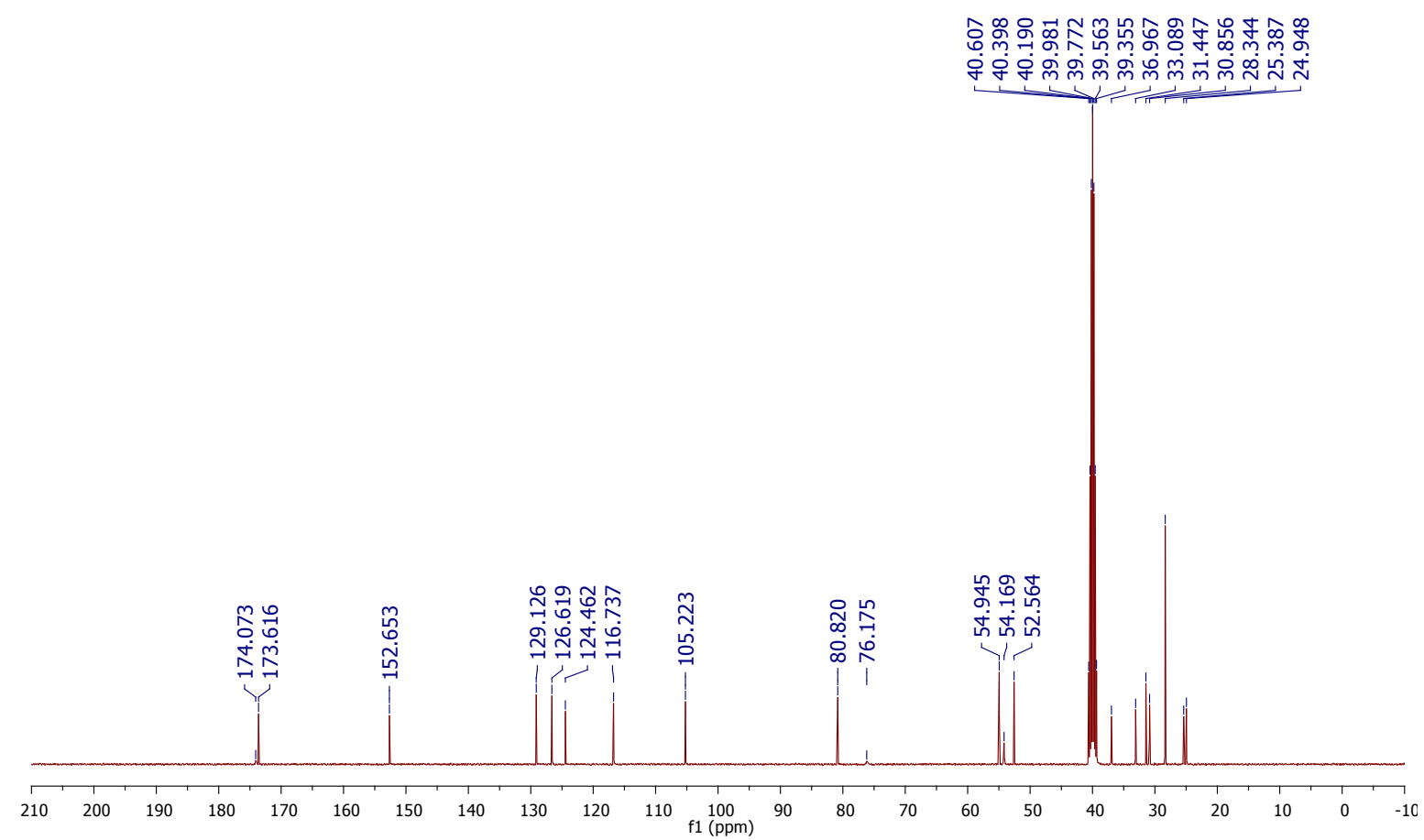


${ }^{1} \mathrm{H}$ NMR of $3 \mathrm{~h}$ (400 MHz, DMSO- $\left.d_{6}\right)$

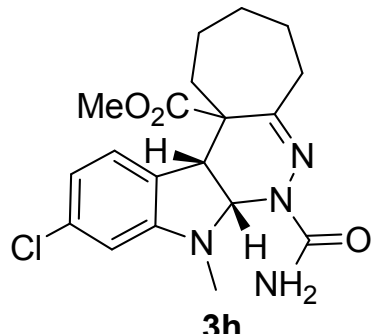

$3 h$
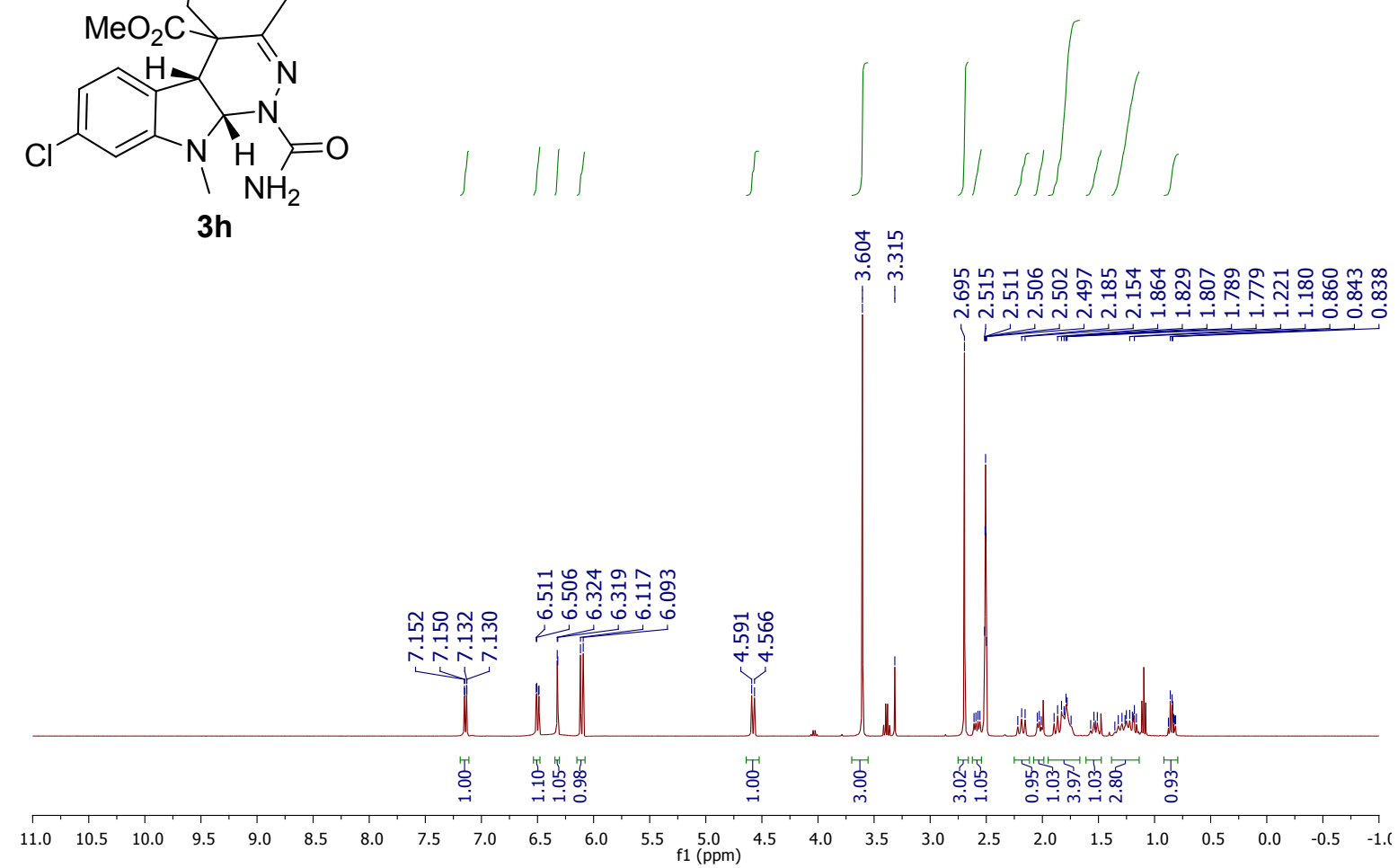

${ }^{13} \mathrm{C}$ NMR of $3 \mathrm{~h}$ (100 MHz, DMSO- $\left.d_{6}\right)$

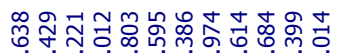

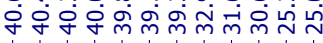

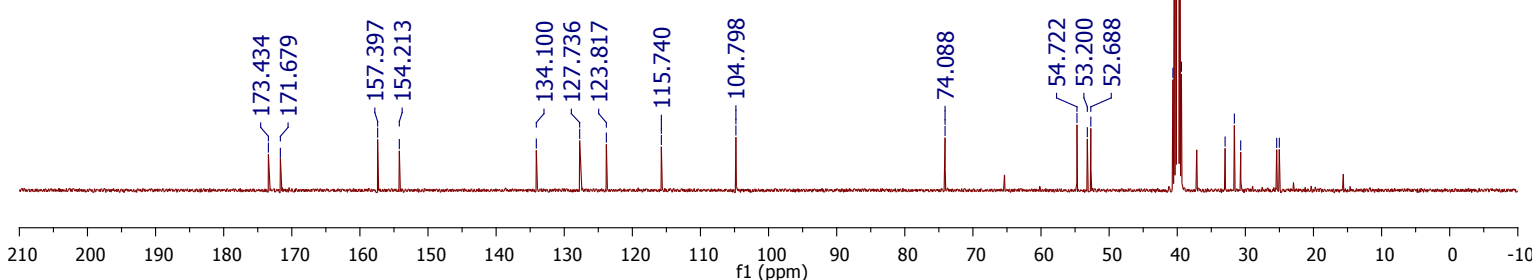


NOESY NMR of $3 \mathrm{~h}\left(400 \mathrm{MHz}\right.$, DMSO- $\left.d_{6}\right)$

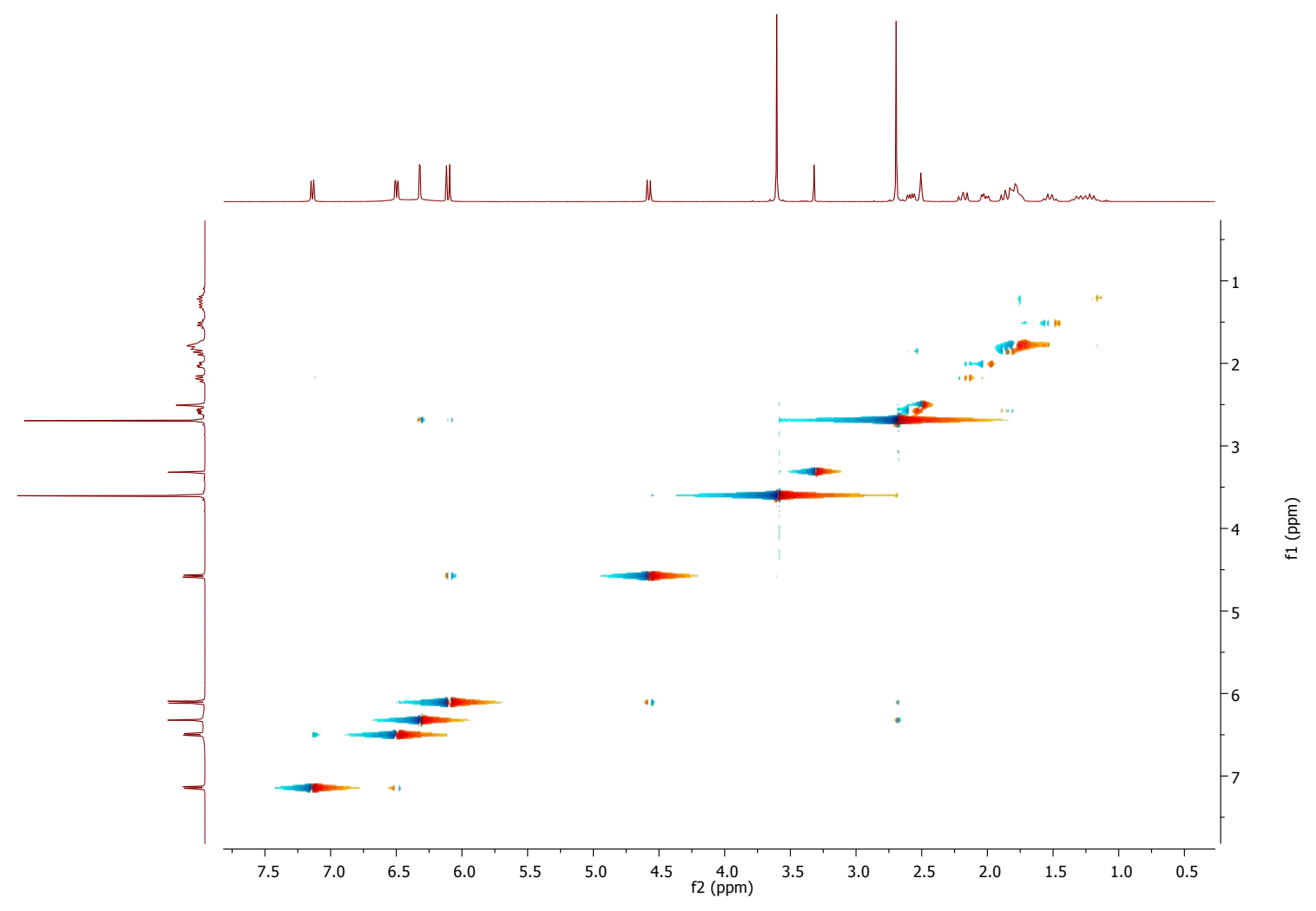


${ }^{1} \mathrm{H}$ NMR of 3i (400 MHz, DMSO- $d_{6}$ )<smiles>COC1(C)[C@H]2N(C)c3c(C)cccc3[C@@]2(C)N(C(N)=O)N=C2CCCCCC21C</smiles>

$3 \mathbf{i}$

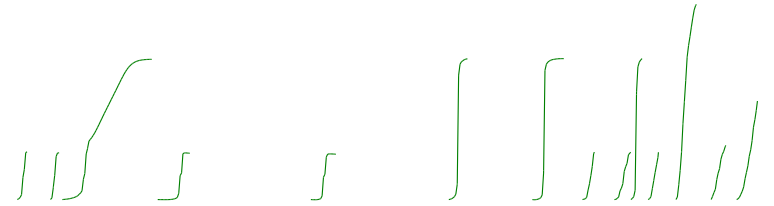

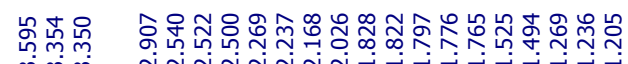

${ }^{13} \mathrm{C}$ NMR of $3 \mathrm{i}$ (100 MHz, DMSO- $\left.d_{6}\right)$

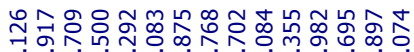

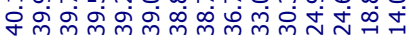

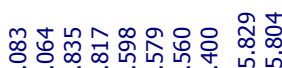
- $0000 \dot{0} \dot{0}$

iil
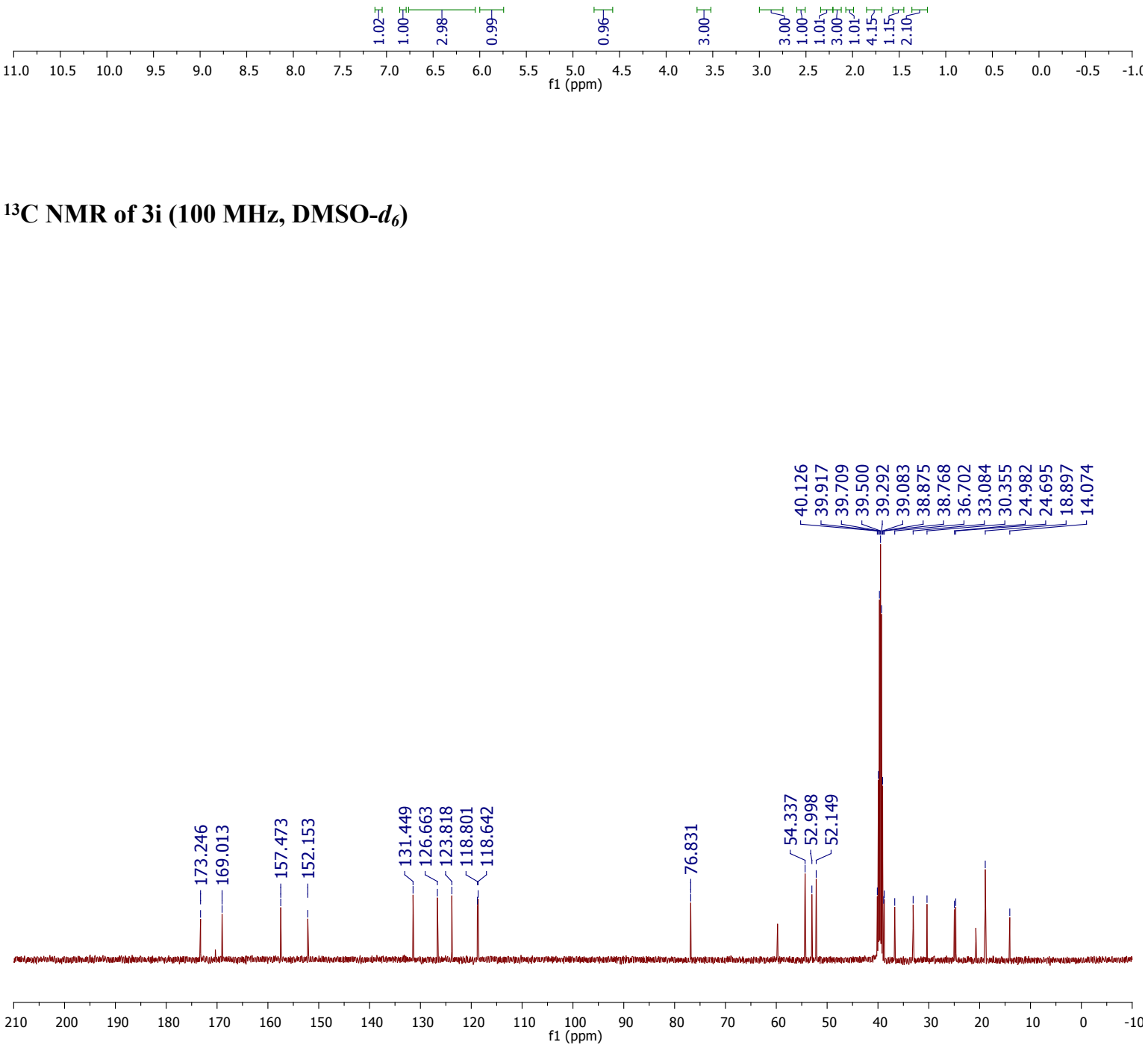
${ }^{1} \mathrm{H}$ NMR of $3 \mathrm{j}$ (400 MHz, DMSO- $\left.d_{6}\right)$

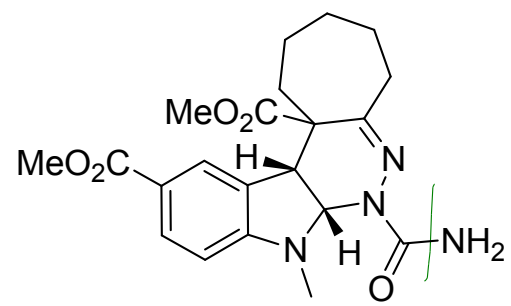

3j

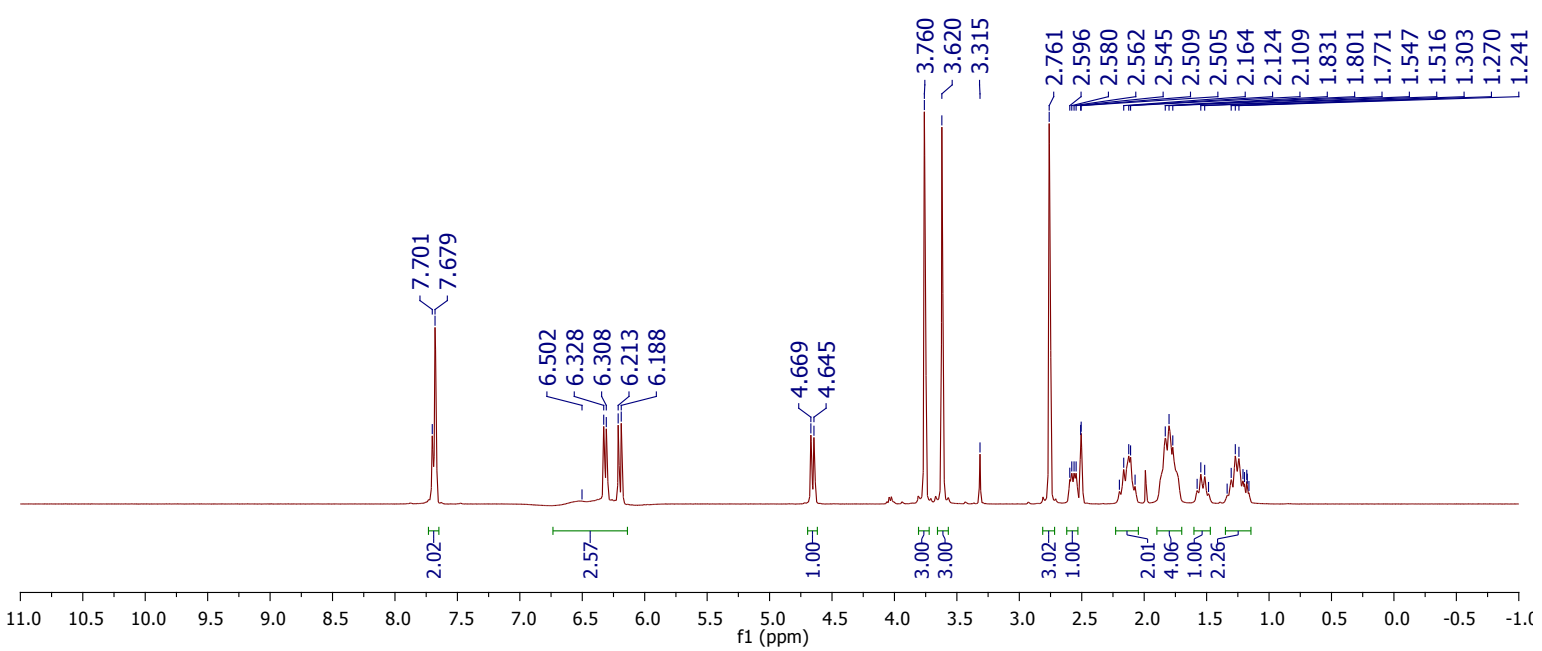

${ }^{13} \mathrm{C}$ NMR of $3 \mathrm{j}$ (100 MHz, DMSO- $\left.d_{6}\right)$

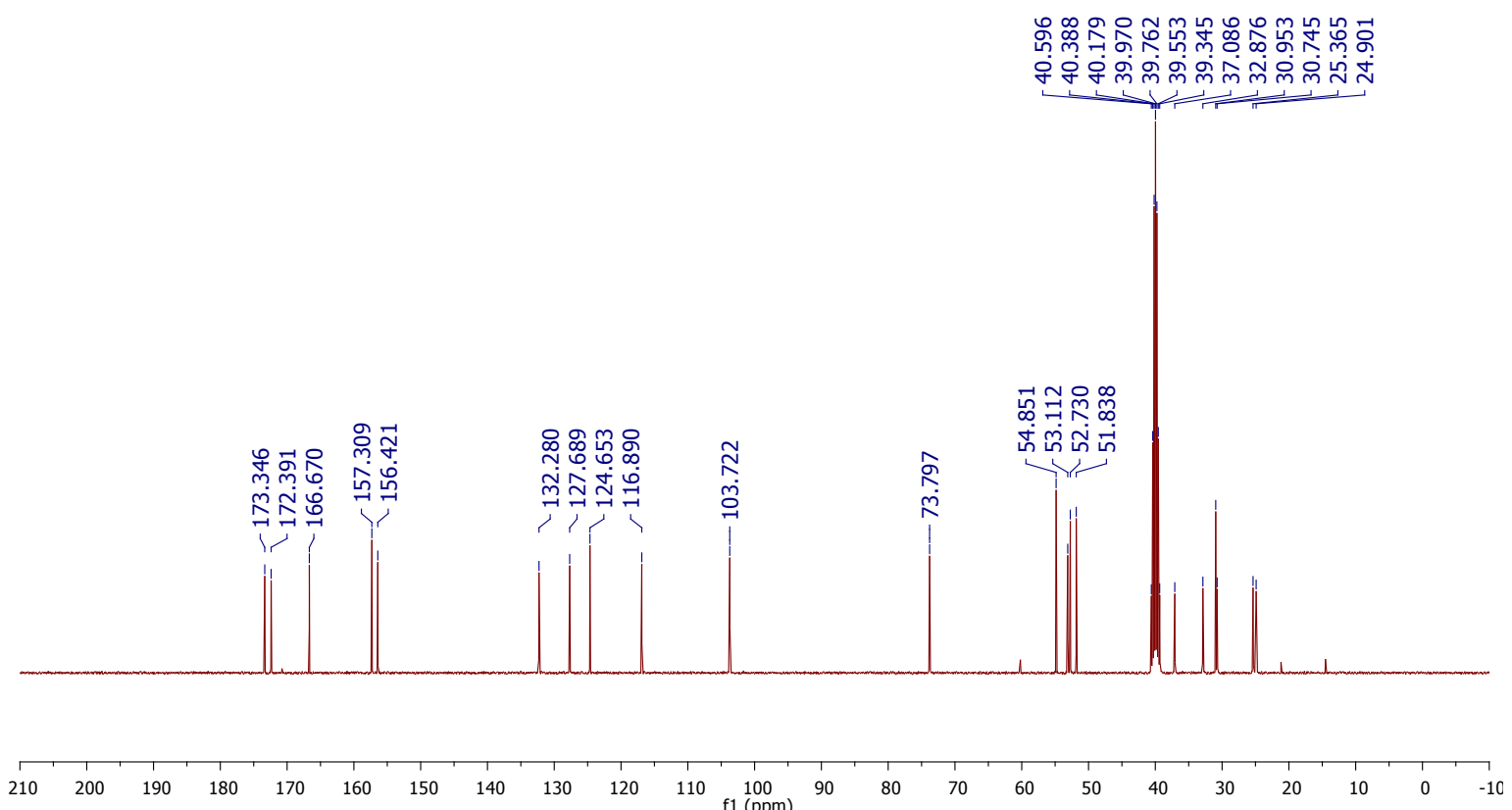


${ }^{1} \mathrm{H}$ NMR of 3k (400 MHz, DMSO- $\left.d_{6}\right)$

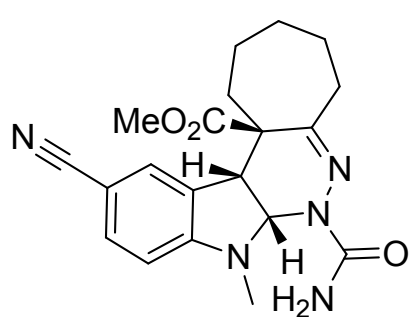

3k

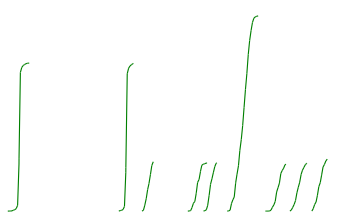

$\begin{array}{lll}0 & 0 \\ \overrightarrow{0} & \stackrel{m}{m} \\ \dot{m} & \stackrel{m}{m}\end{array}$

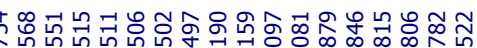

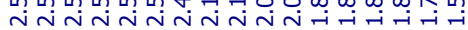

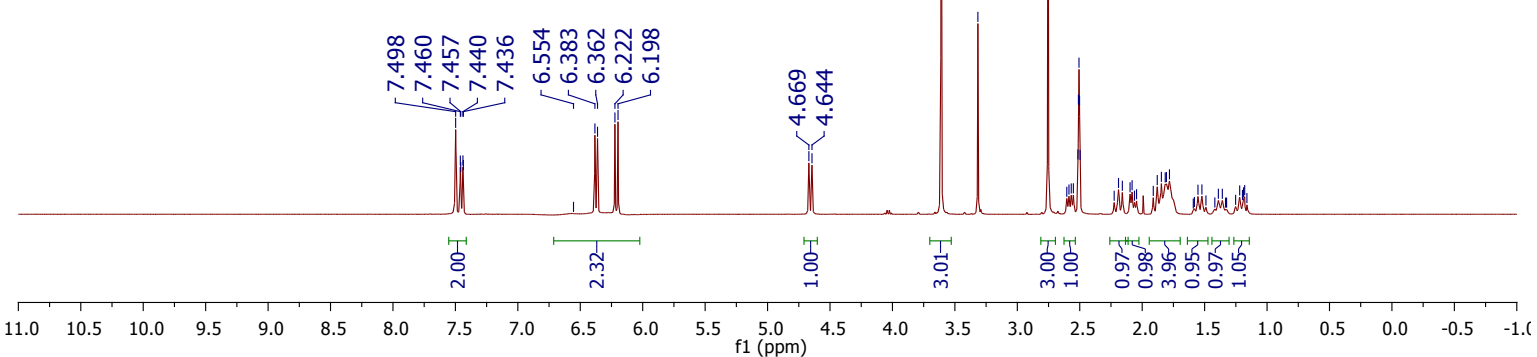

${ }^{13} \mathrm{C}$ NMR of 3k (100 MHz, DMSO-d $)$

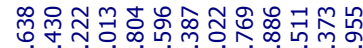

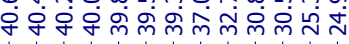
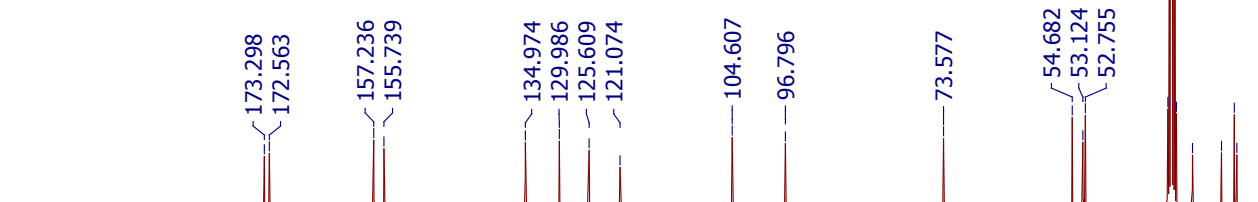

$\begin{array}{lllllllllll}210 & 200 & 190 & 180 & 170 & 160 & 150 & 140 & 130 & 120 & 110 \\ \mathrm{f} 1(\mathrm{ppm})\end{array}$ 
${ }^{1} \mathrm{H}$ NMR of 31 (400 MHz, DMSO- $\left.d_{6}\right)$

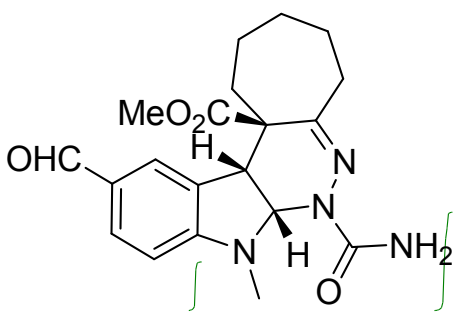

3I

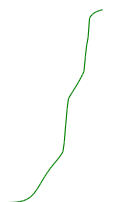

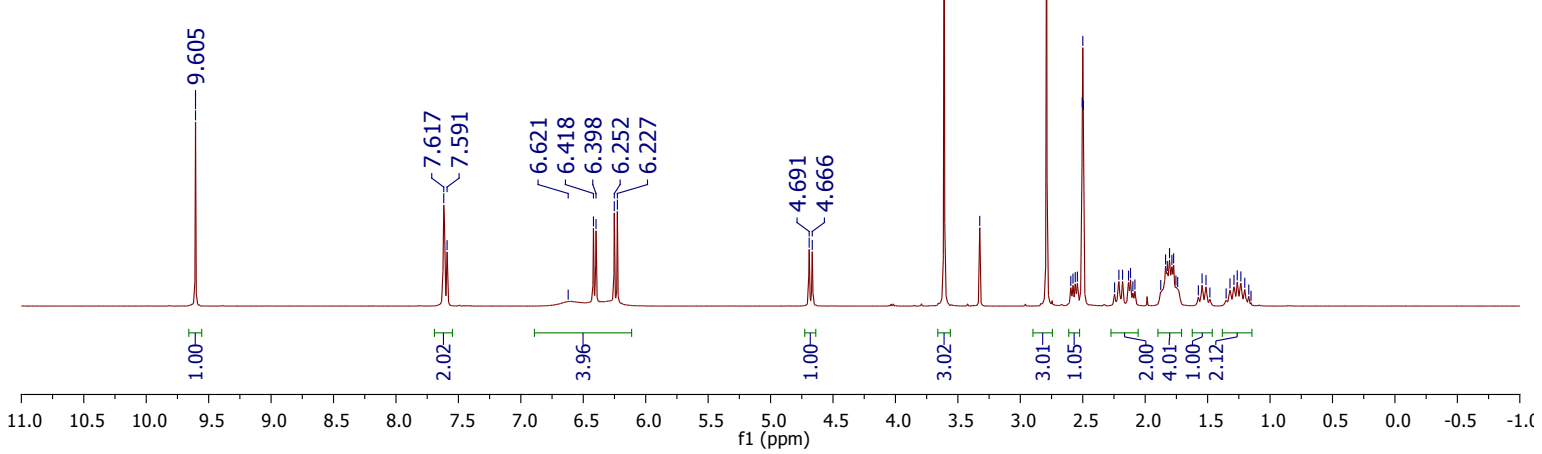

${ }^{13} \mathrm{C}$ NMR of 31 (100 MHz, DMSO- $\left.d_{6}\right)$

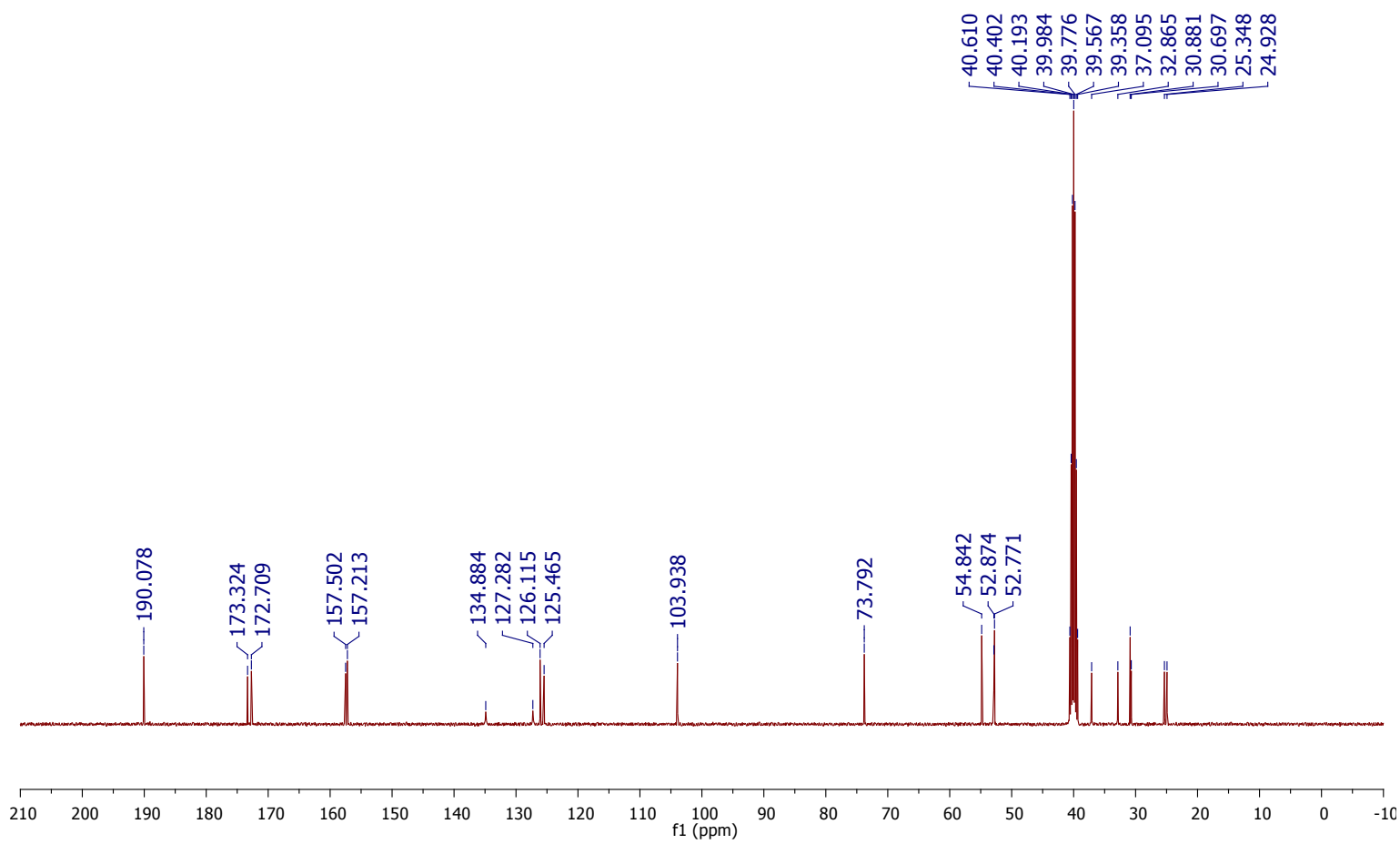


${ }^{1} \mathrm{H}$ NMR of 3m (400 MHz, DMSO- $\left.d_{6}\right)$

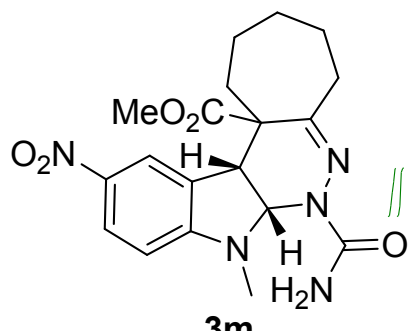

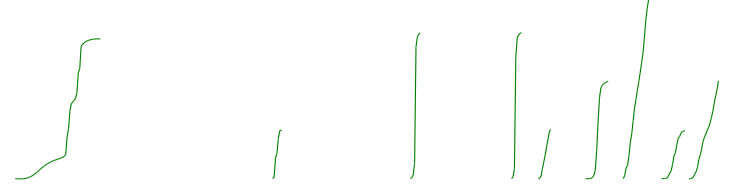

$3 \mathrm{~m}$

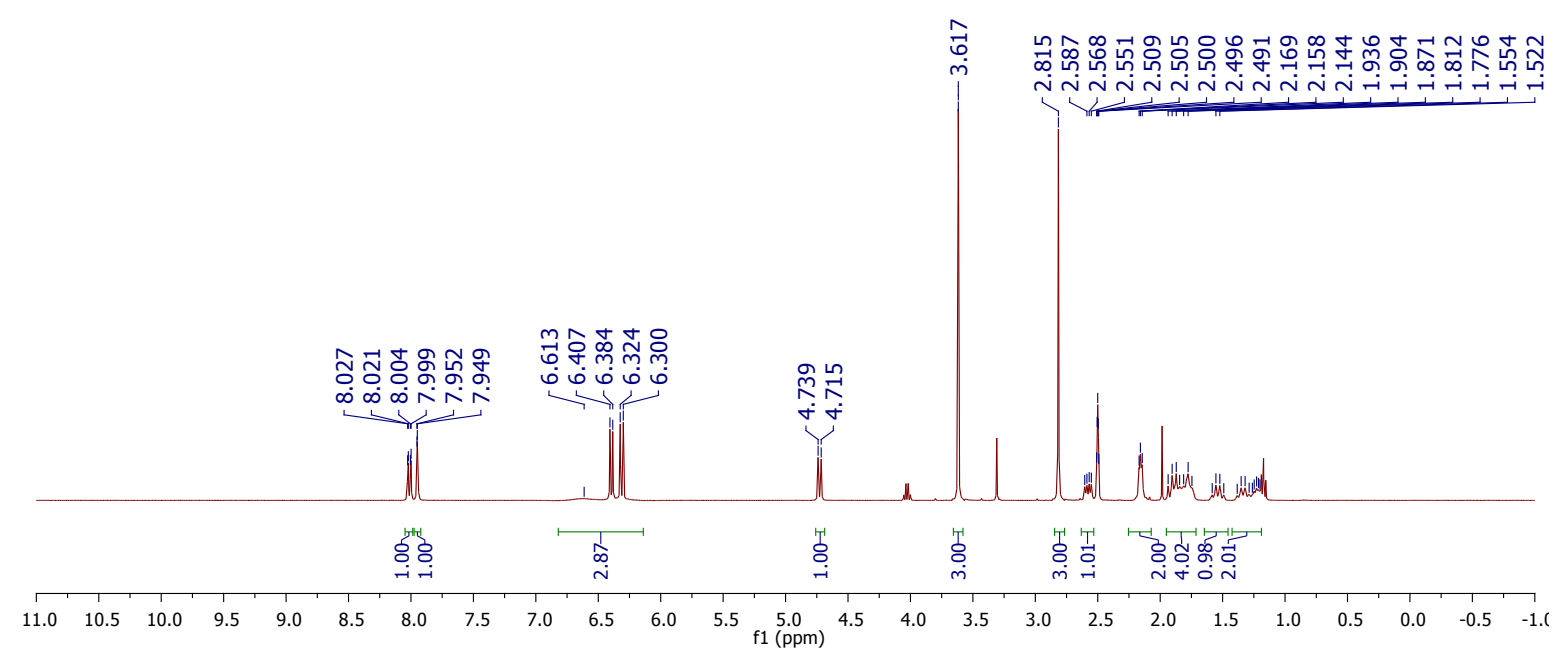

${ }^{13} \mathrm{C}$ NMR of 3m (100 MHz, DMSO- $\left.d_{6}\right)$

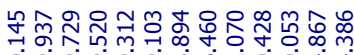

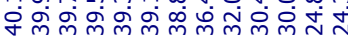

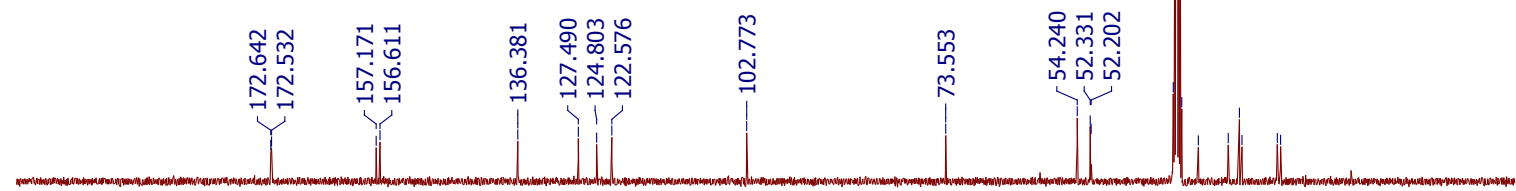

$\begin{array}{llllllllllllllllllllllllllll}210 & 200 & 190 & 180 & 170 & 160 & 150 & 140 & 130 & 120 & 110 & 100 & 90 & 80 & 70 & 60 & 50 & 40 & 30 & 20 & 10 & 0 & -10\end{array}$ 
${ }^{1} \mathrm{H}$ NMR of 3n (400 MHz, DMSO-d $\left.)_{6}\right)$<smiles>COc1ccc2c(c1)[C@@]1(C)C3(C)CCCCCC3=NN(C(N)=O)[C@@]1(C)N(Cc1ccccc1)C2=O</smiles>

$3 n$

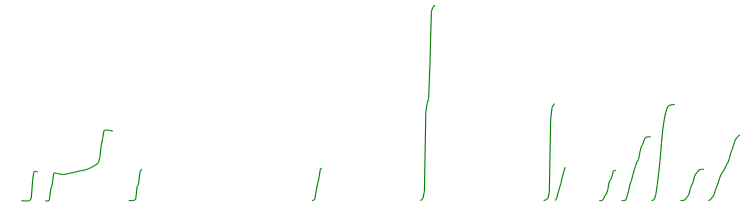

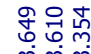

$m m$

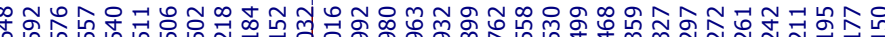

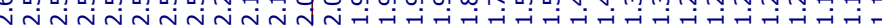

${ }^{13}$ C NMR of 3n (100 MHz, DMSO- $\left.d_{6}\right)$

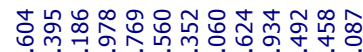

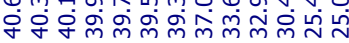

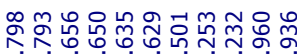

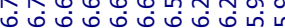

I.

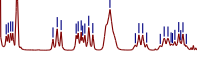

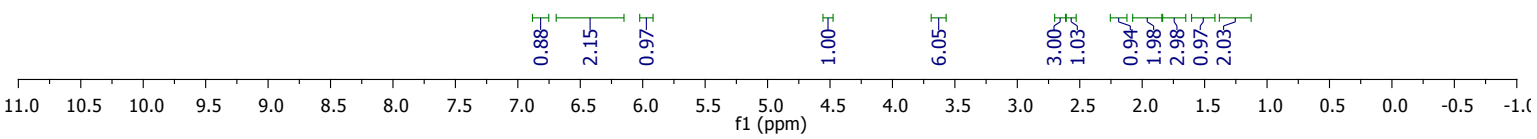

등ำ

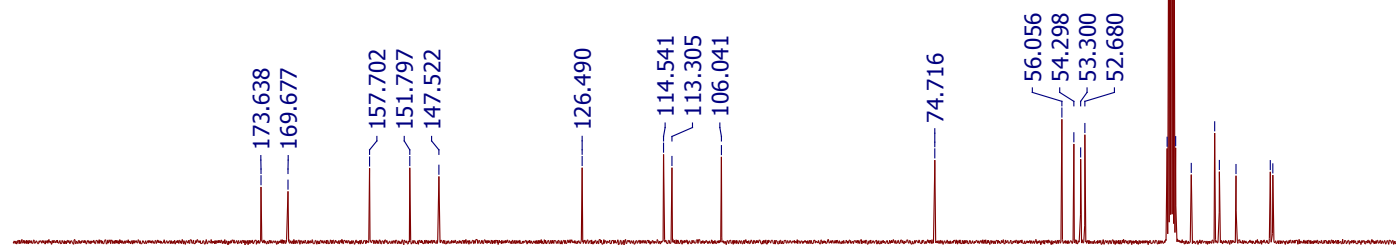


${ }^{1} \mathrm{H}$ NMR of 30 (400 MHz, DMSO- $d_{6}$ )

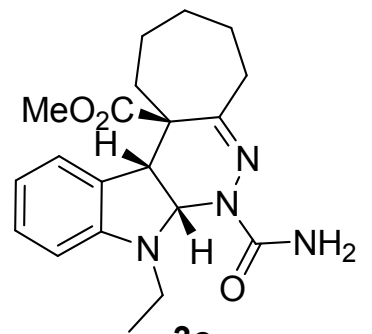

30<smiles>C#CCCCCCCC</smiles>

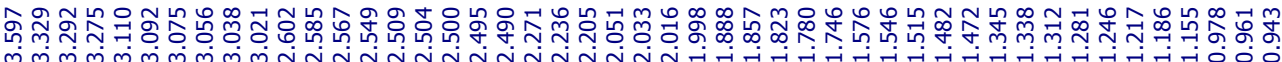

${ }^{13}$ C NMR of 30 (100 MHz, DMSO- $\left.d_{6}\right)$

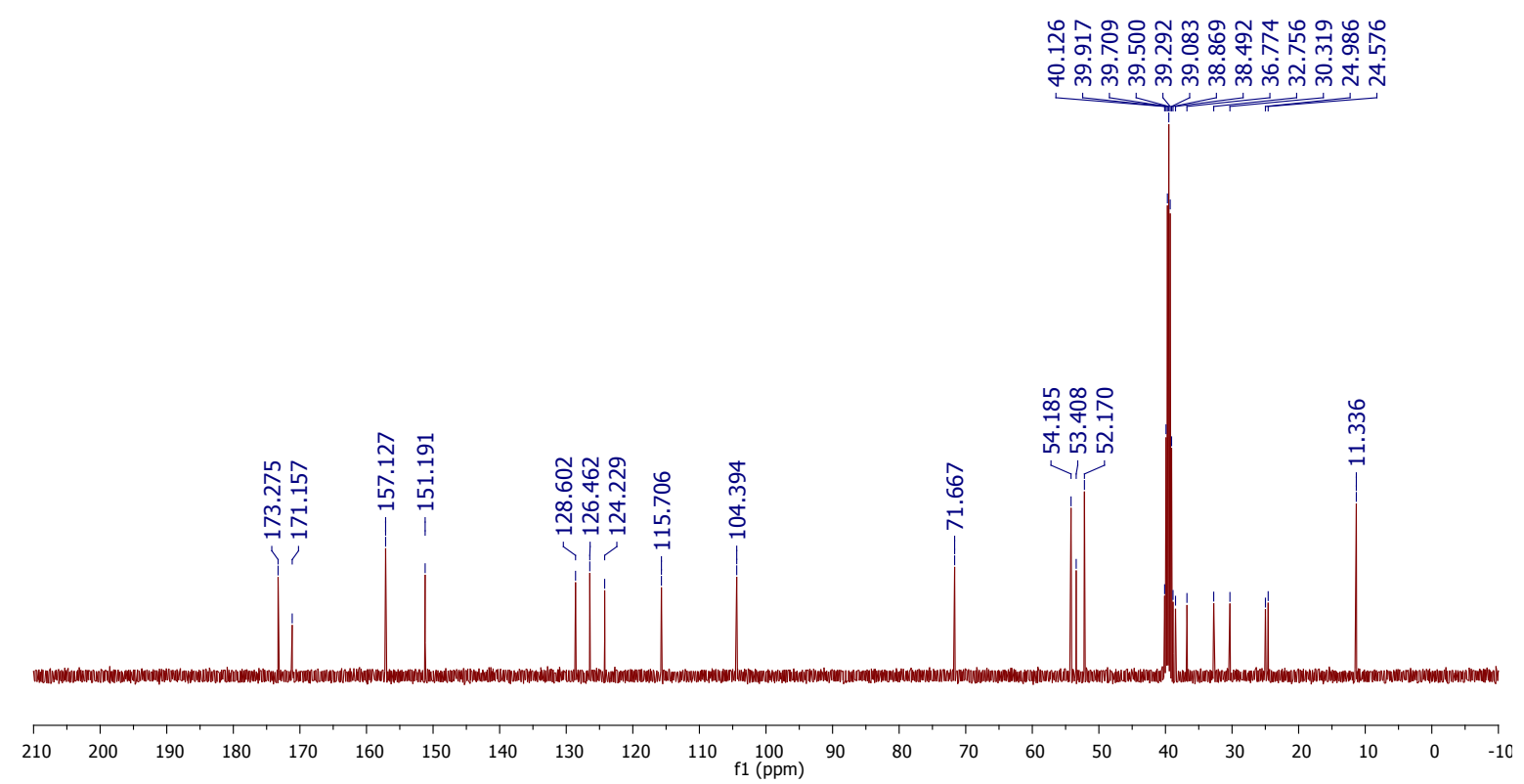


${ }^{1} \mathrm{H}$ NMR of 3p (400 MHz, DMSO-d $\left._{6}\right)$<smiles>CCCCCCC12CCCCCC1(C)c1cc(C)ccc1N2CCC</smiles><smiles>C=CCCC</smiles>

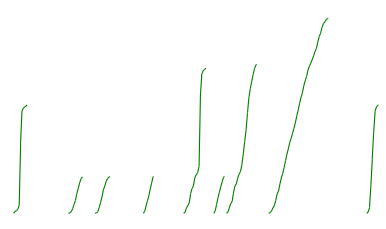

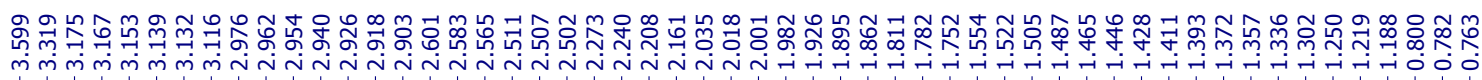

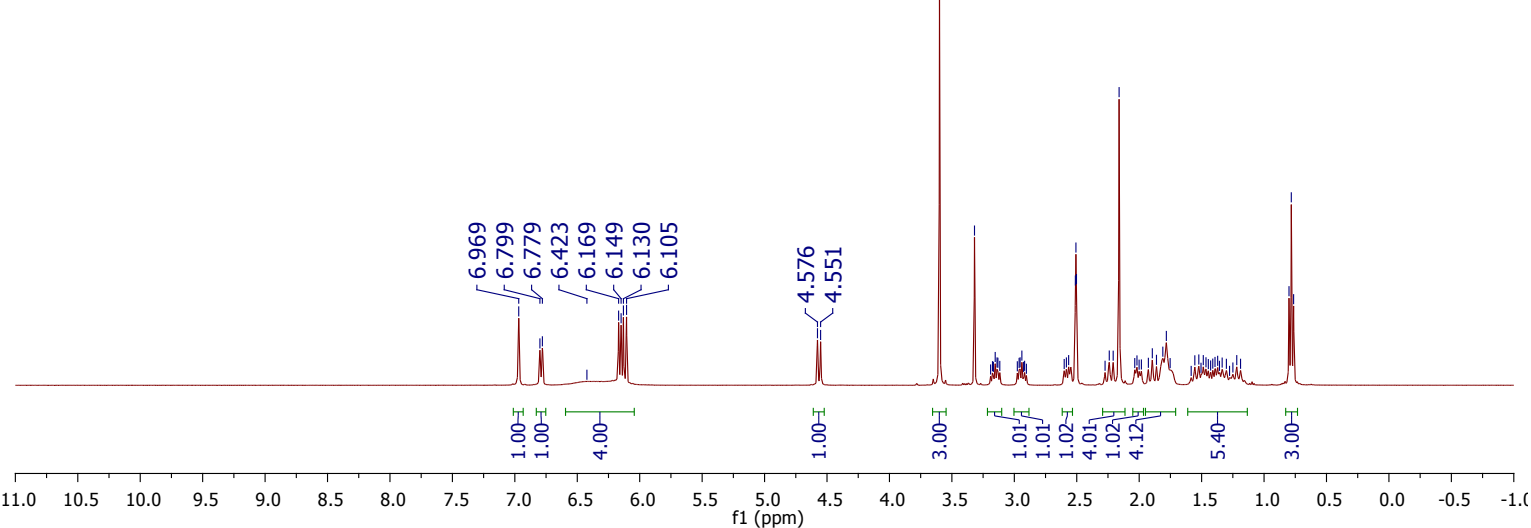

${ }^{13} \mathrm{C}$ NMR of 3p (100 MHz, DMSO- $\left.d_{6}\right)$

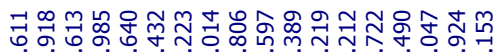

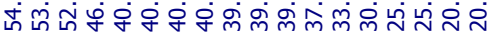

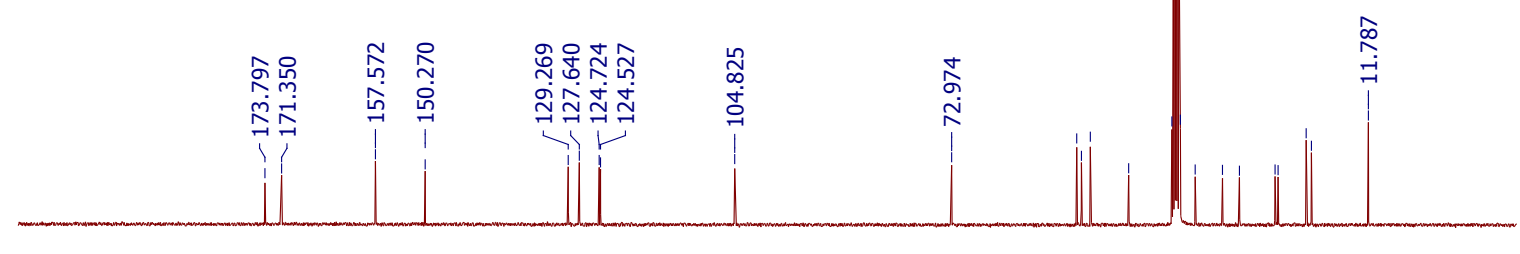

$\begin{array}{lllllllllllllllllllllllllllll}210 & 200 & 190 & 180 & 170 & 160 & 150 & 140 & 130 & 120 & 110 & 100 & 90 & 80 & 70 & 60 & 50 & 40 & 30 & 20 & 10 & 0 & -10\end{array}$ 
${ }^{1} \mathrm{H}$ NMR of 3q (400 MHz, DMSO-d $\left.{ }_{6}\right)$<smiles>COC1(C)[C@@]2(C)N(Cc3ccccc3)c3ccccc3[C@@]2(C)N(C(N)=O)N=C2CCCCC[C@]21C</smiles>
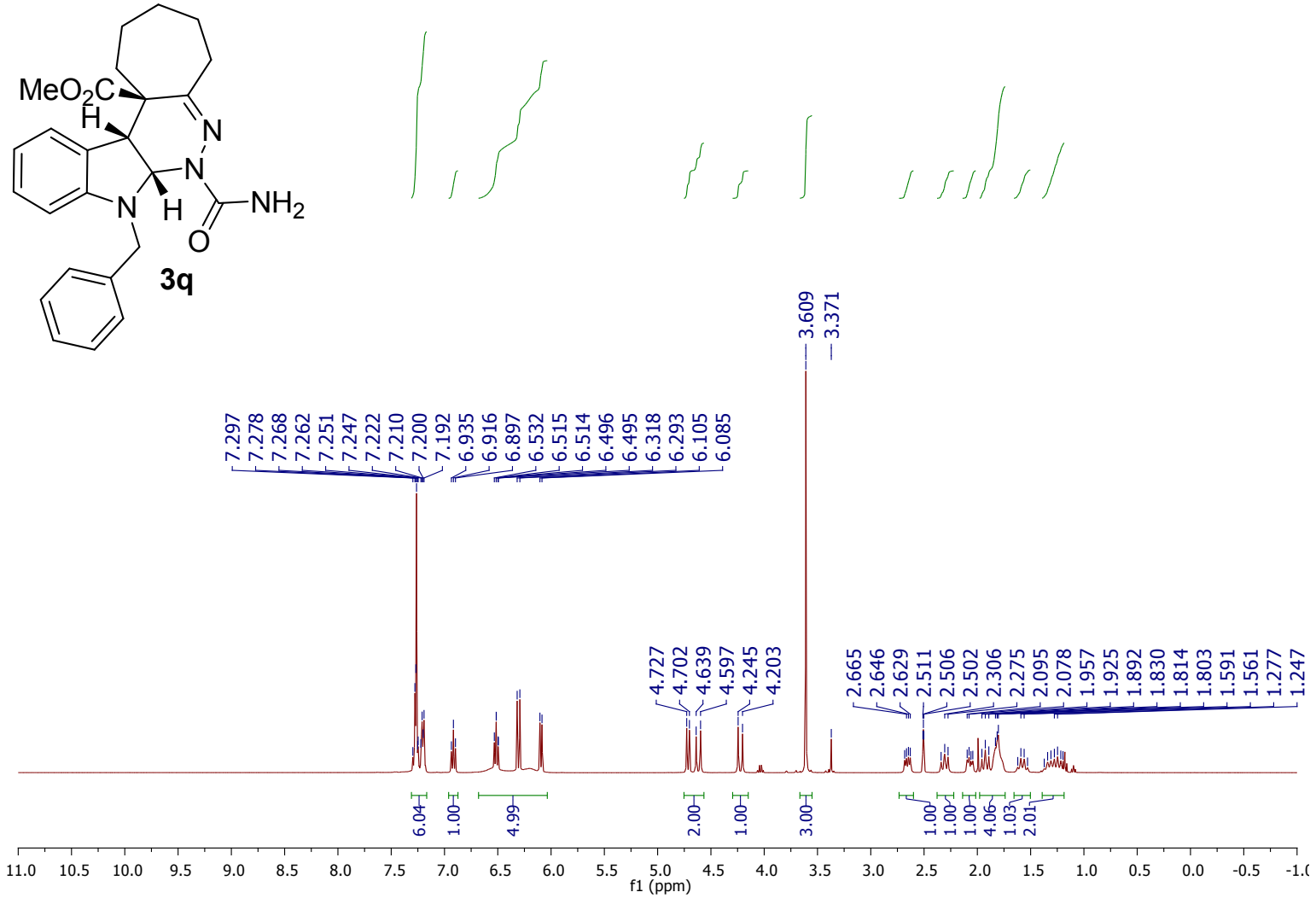

${ }^{13} \mathrm{C}$ NMR of 3q (100 MHz, DMSO- $\left.d_{6}\right)$

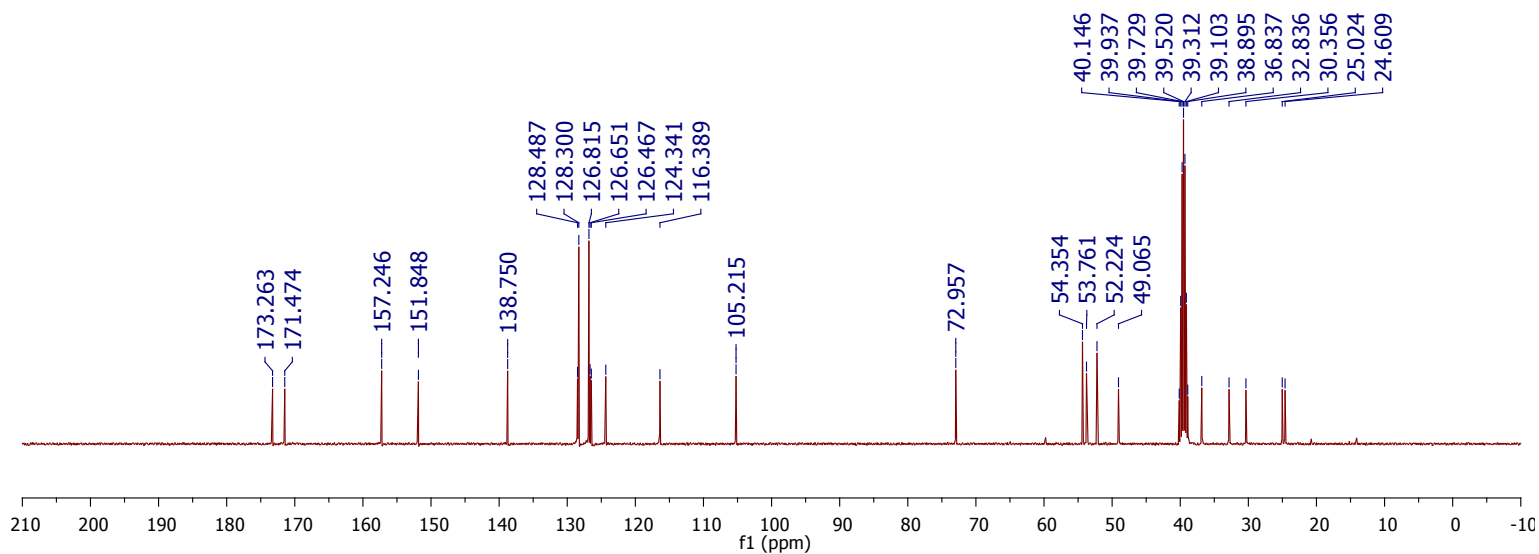


${ }^{1} \mathrm{H}$ NMR of $3 \mathrm{r}$ (400 MHz, DMSO- $\left.d_{6}\right)$

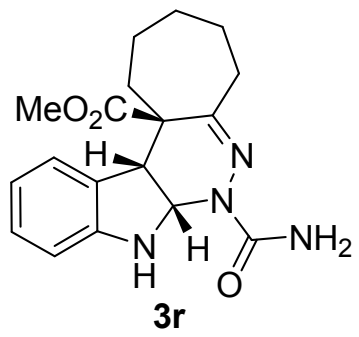

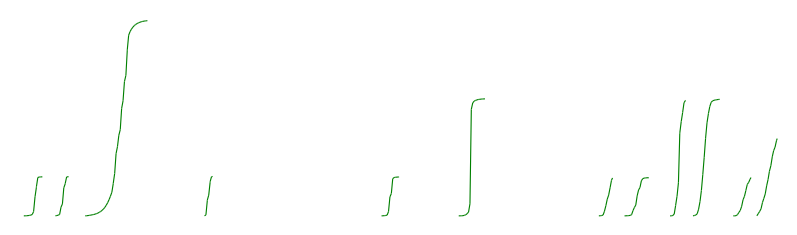

$3 r$

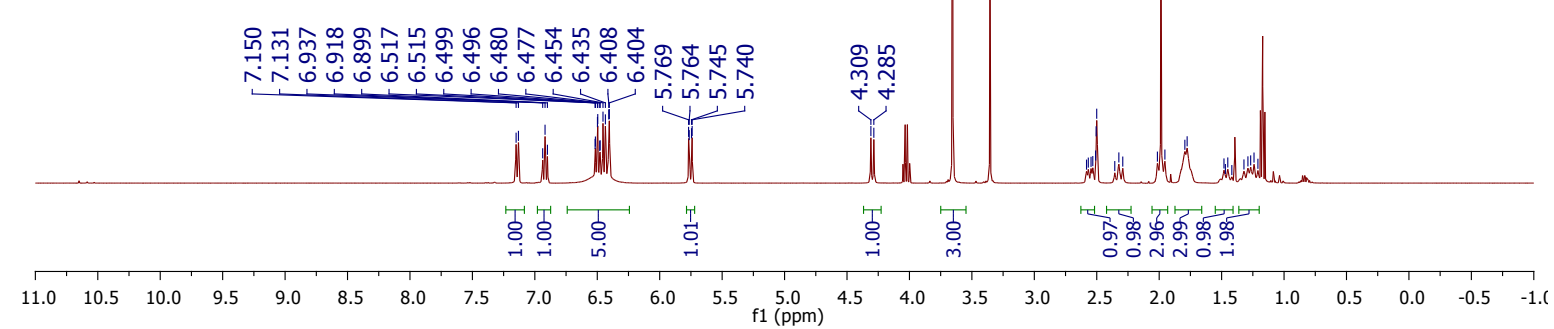

${ }^{13} \mathrm{C}$ NMR of $3 \mathrm{r}\left(100 \mathrm{MHz}\right.$, DMSO- $\left.d_{6}\right)$

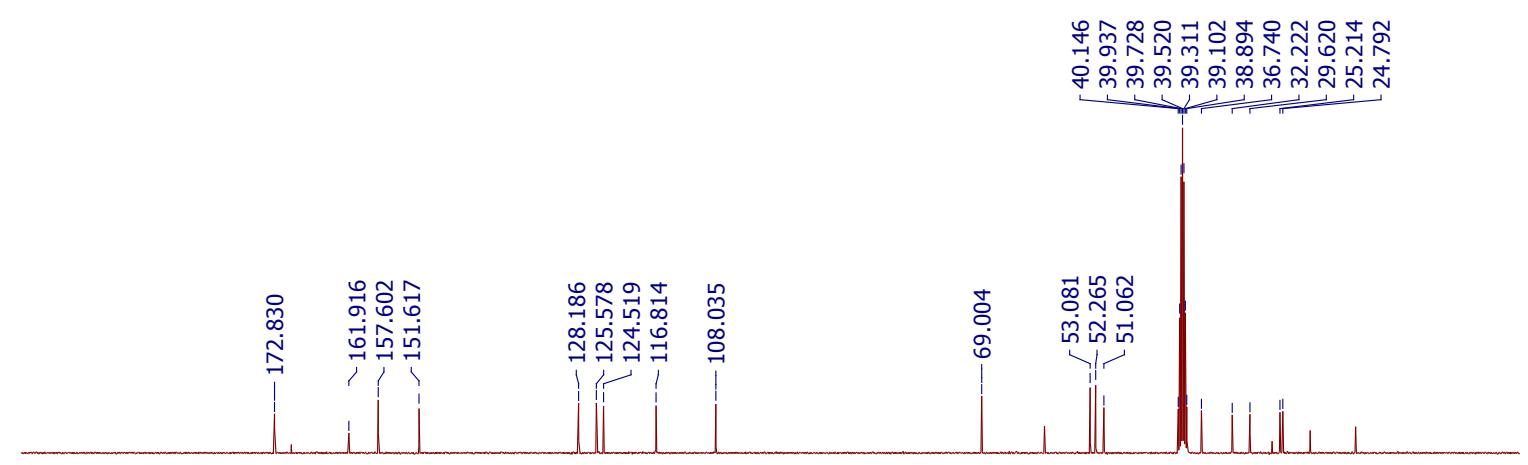

$\begin{array}{llllllllllllllllllllllllll}210 & 200 & 190 & 180 & 170 & 160 & 150 & 140 & 130 & 120 & 110 & 100 & 90 & 80 & 70 & 60 & 50 & 40 & 30 & 20 & 10 & 0\end{array}$ 
${ }^{1}$ H NMR of 3s (400 MHz, DMSO- $d_{6}$ )<smiles>COC(=O)[C@]12CCCCCC1=NN(C(N)=O)C1(C2)Nc2ccc(C)cc2[C@H]1C(C)(C)C</smiles>

3s
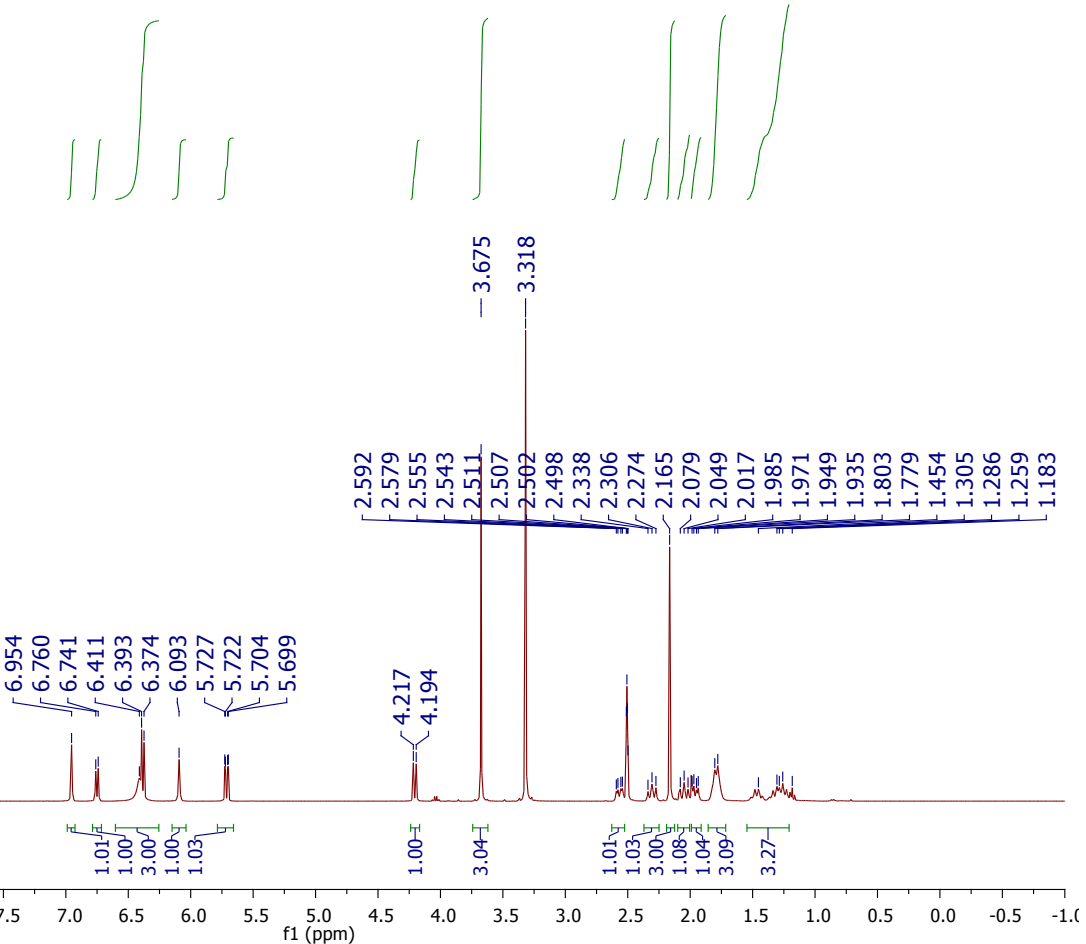

${ }^{13} \mathrm{C}$ NMR of 3s (100 MHz, DMSO- $d_{6}$ )

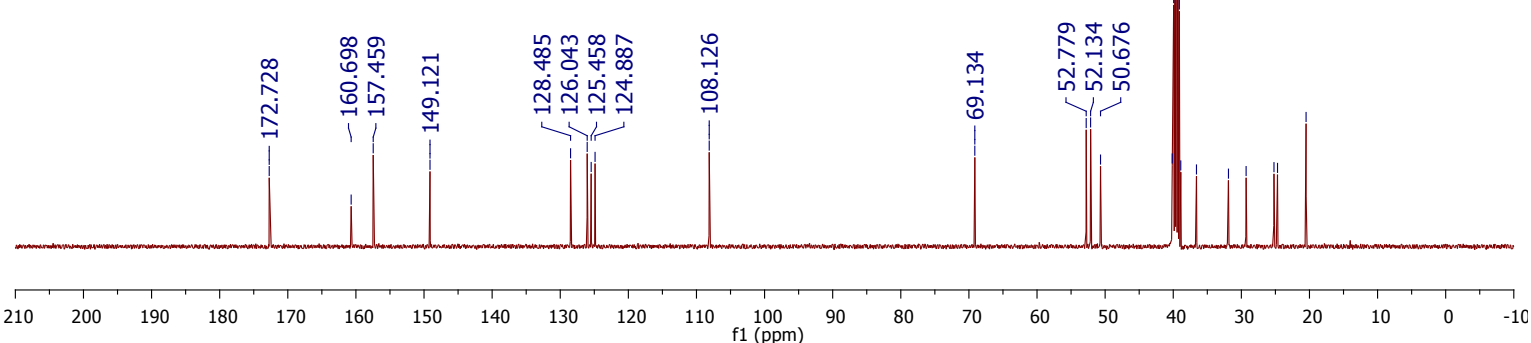


${ }^{1} \mathrm{H}$ NMR of $3 t$ (400 MHz, DMSO- $\left.d_{6}\right)$

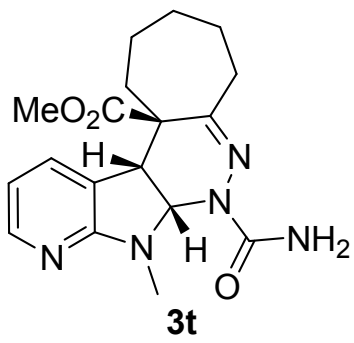

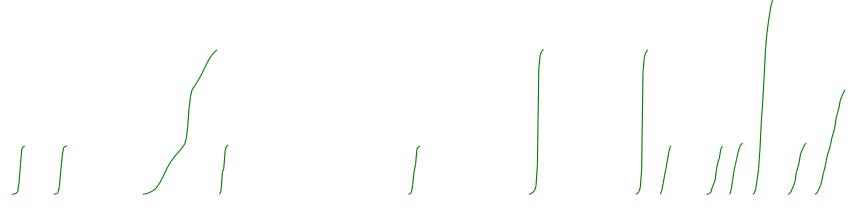

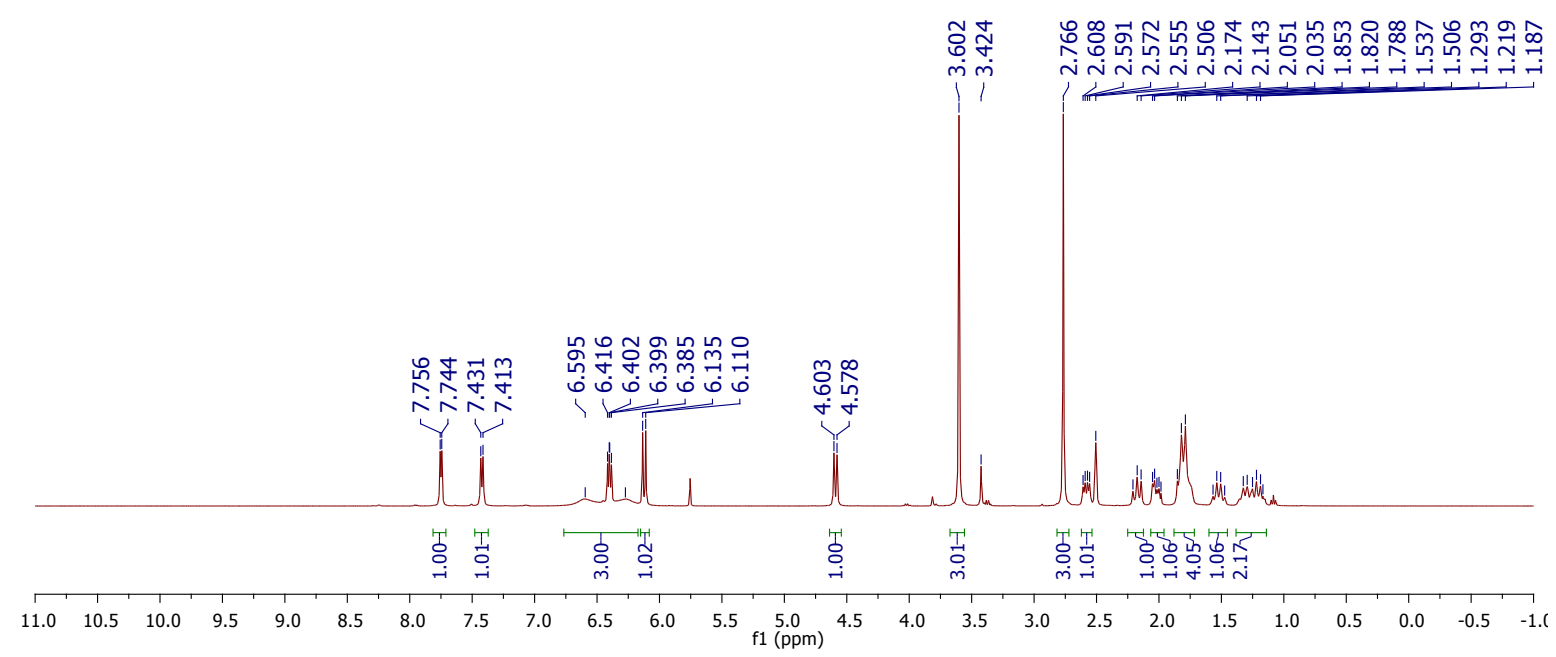

${ }^{13} \mathrm{C}$ NMR of $3 \mathrm{t}$ (100 MHz, DMSO- $\left.d_{6}\right)$

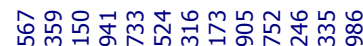

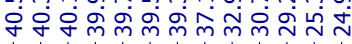

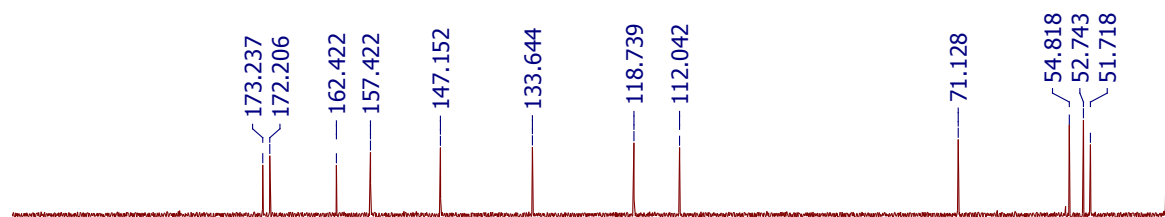

$\begin{array}{lllllllllllllllllllllllllllll}210 & 200 & 190 & 180 & 170 & 160 & 150 & 140 & 130 & 120 & 110 & 100 & 90 & 80 & 70 & 60 & 50 & 40 & 30 & 20 & 10 & 0 & -10\end{array}$ 
${ }^{1} \mathrm{H}$ NMR of $3 \mathrm{u}\left(400 \mathrm{MHz}\right.$, DMSO- $\left.d_{6}\right)$<smiles>CCOCC12CCCCCCC1=NN(C(N)=O)[C@@]1(C)c3ccccc3N(C)[C@@]21C</smiles>

3u

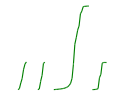

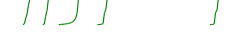

.

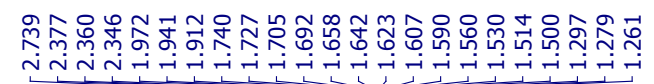

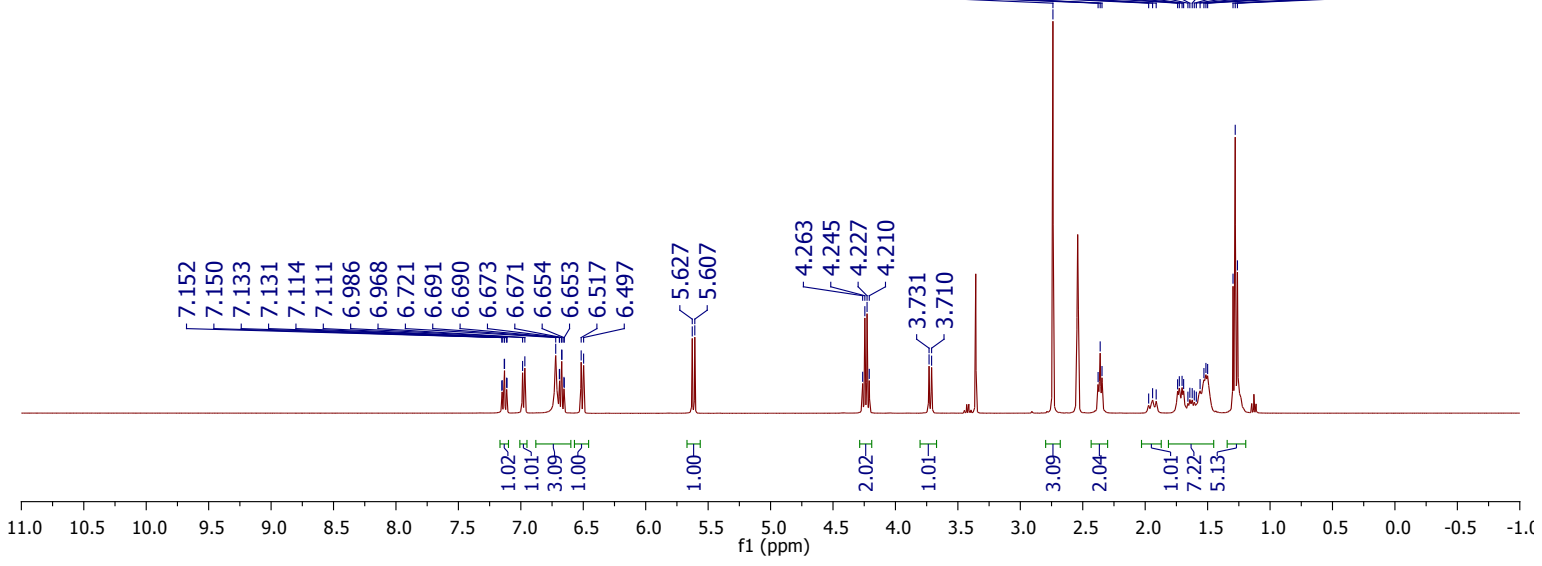

${ }^{13} \mathrm{C}$ NMR of $3 \mathrm{u}\left(100 \mathrm{MHz}\right.$, DMSO- $\left.d_{6}\right)$

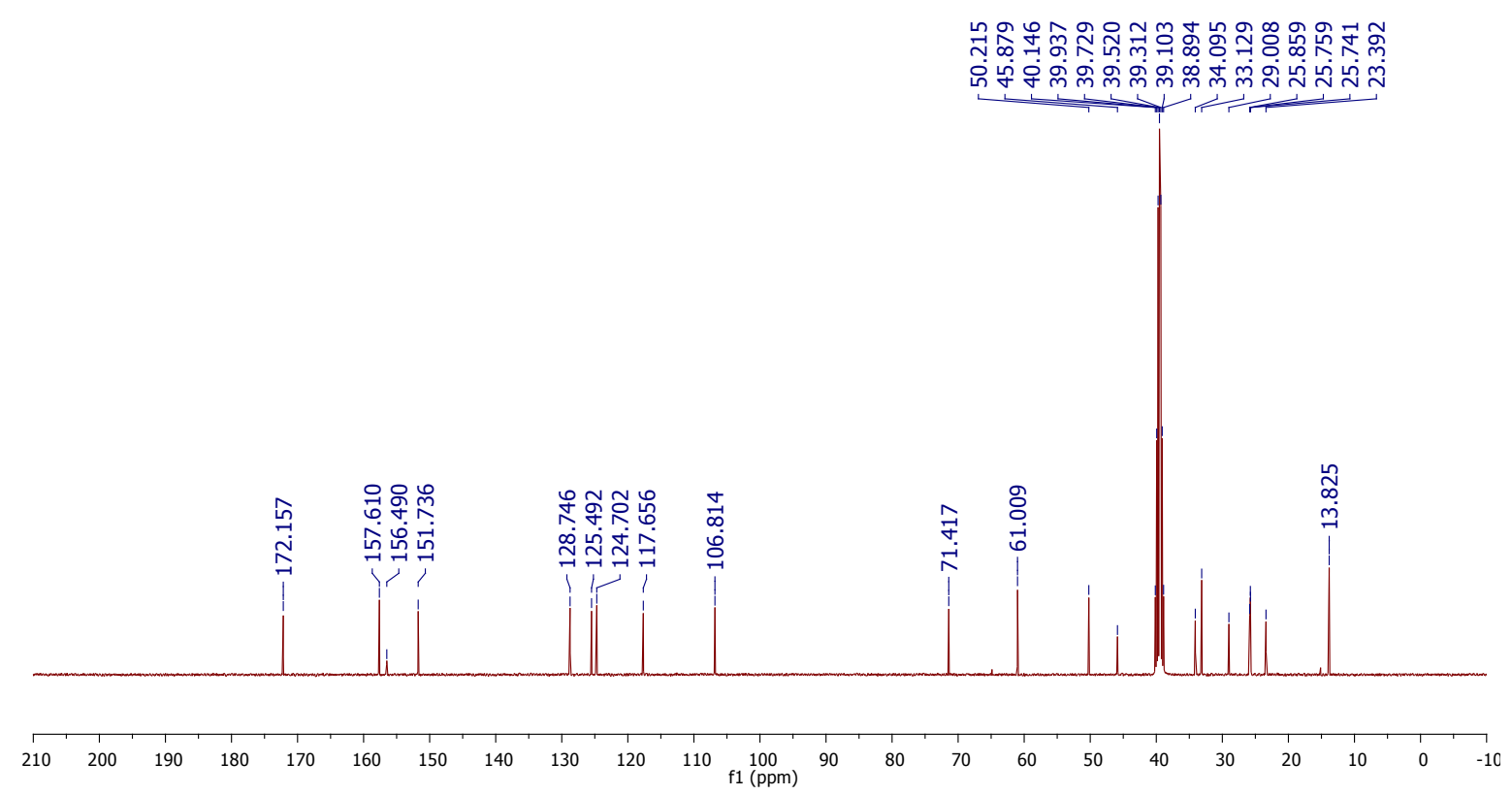


${ }^{1} \mathrm{H}$ NMR of 3v (400 MHz, DMSO- $\left.d_{6}\right)$<smiles>CCOCC[C@]12CCCCCCC1=NN(C(=O)OC(C)(C)C)C1(C)N(C)c3ccccc3[C@@]12C</smiles>

$3 v$

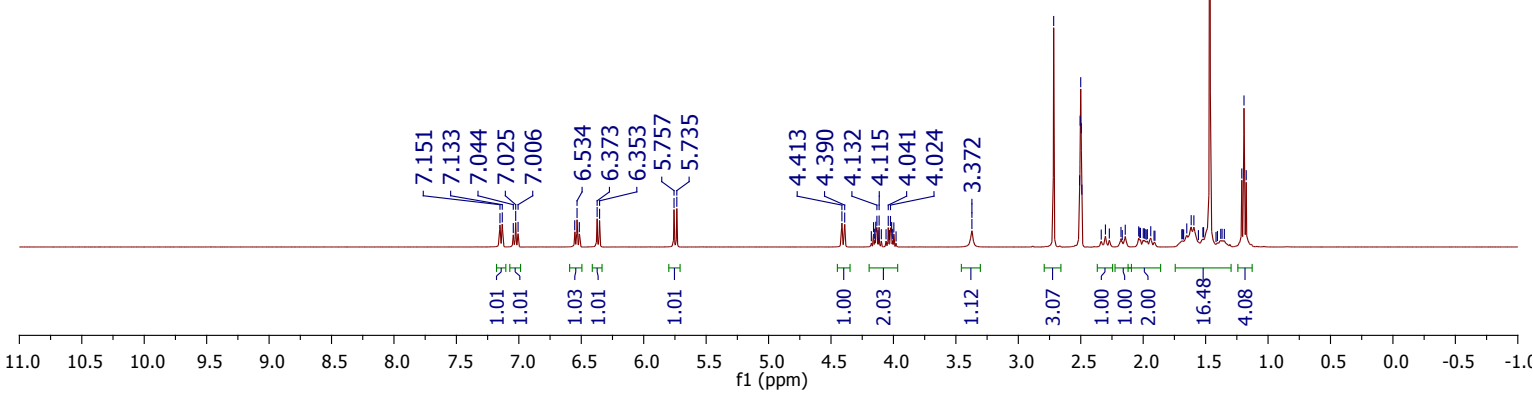

${ }^{13}$ C NMR of 3v (100 MHz, DMSO- $\left.d_{6}\right)$

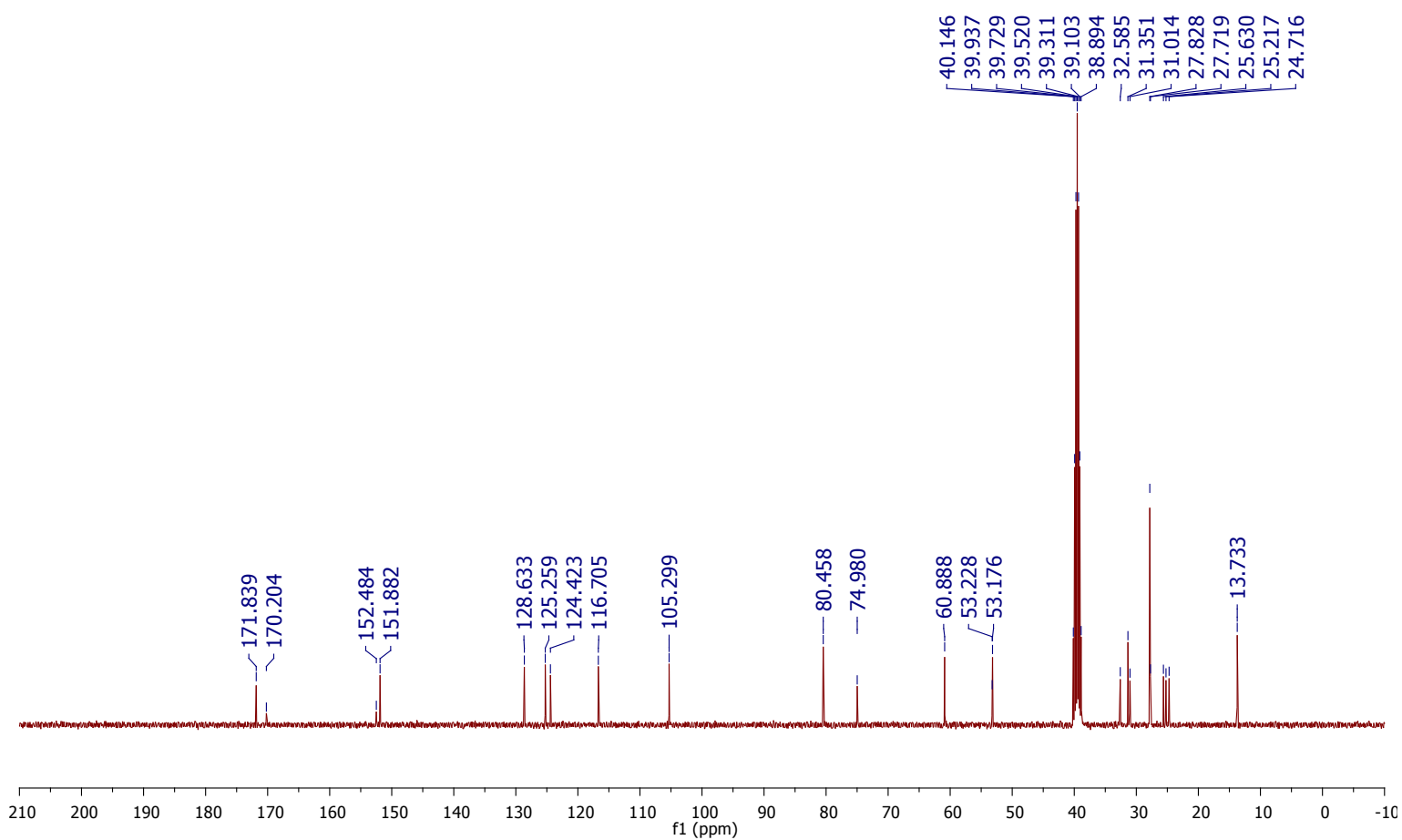


${ }^{1} \mathrm{H}$ NMR of 3w (400 MHz, DMSO- $d_{6}$ )

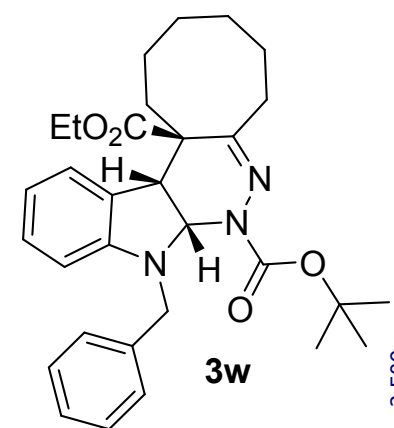

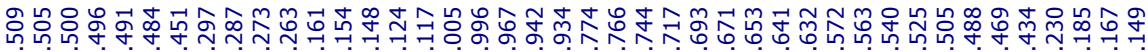

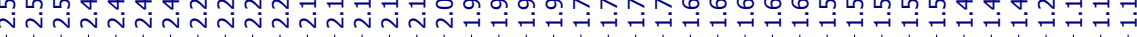

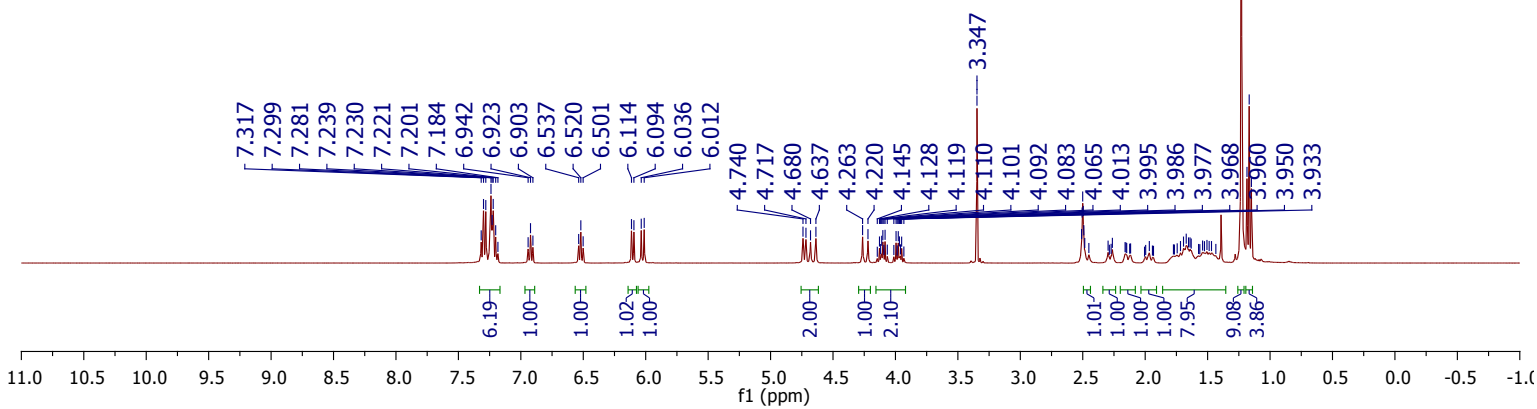

${ }^{13} \mathrm{C}$ NMR of 3w (100 MHz, DMSO- $\left.d_{6}\right)$

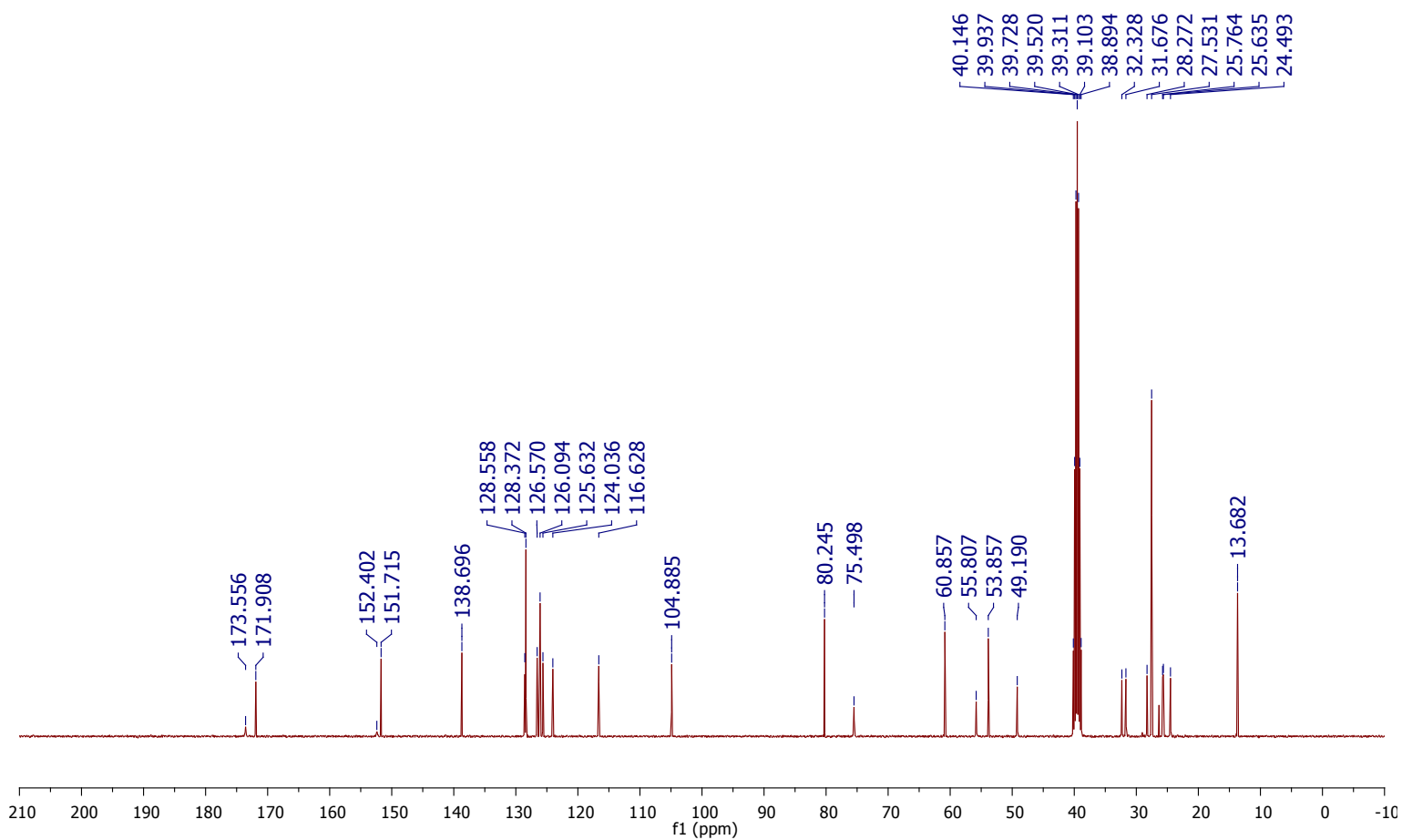


${ }^{1} \mathrm{H}$ NMR of 3x (400 MHz, DMSO- $\left.d_{6}\right)$<smiles>CCOC[C@]12CCCCCCC3=NN(C(N)=O)[C@H](Nc4ccccc4[C@]31C)C2(C)C</smiles>

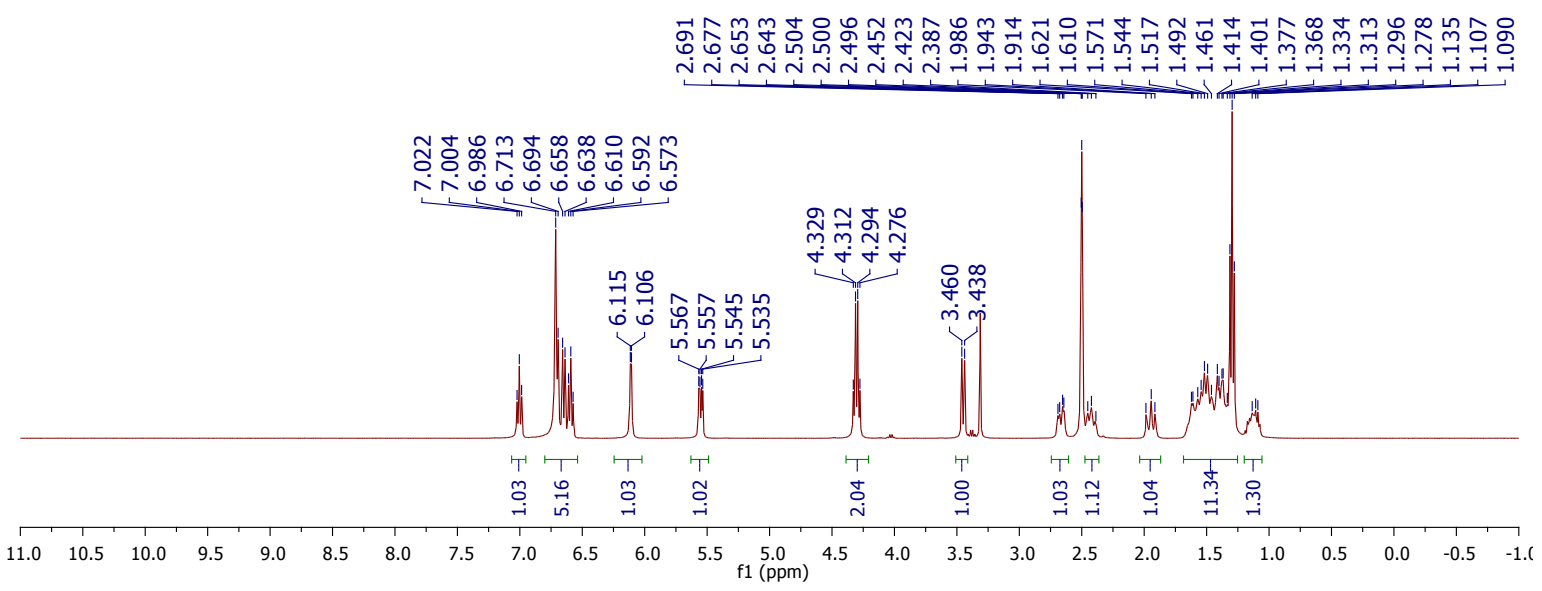

${ }^{13}$ C NMR of 3x (100 MHz, DMSO- $\left.d_{6}\right)$

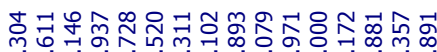

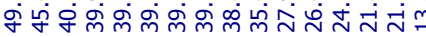

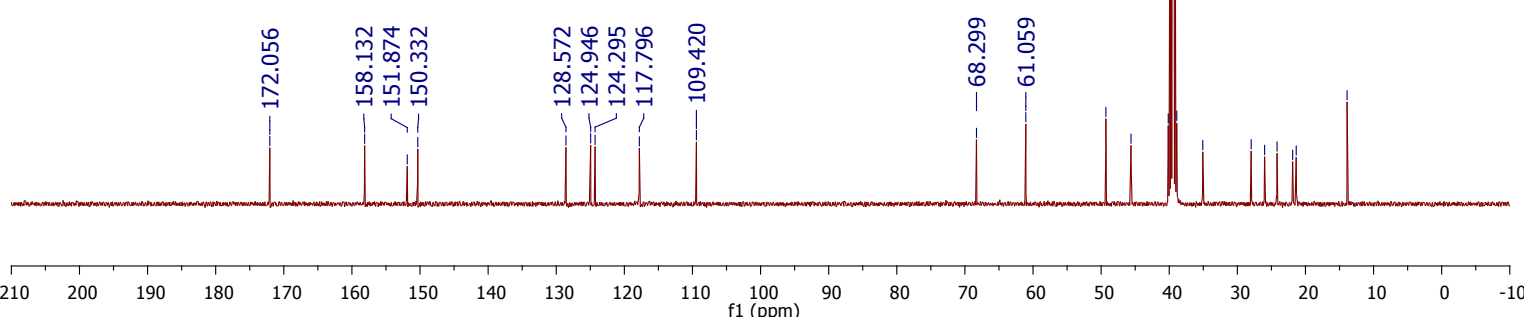


${ }^{1} \mathrm{H}$ NMR of (cis,cis)-3z (400 MHz, DMSO-d $\left.{ }_{6}\right)$

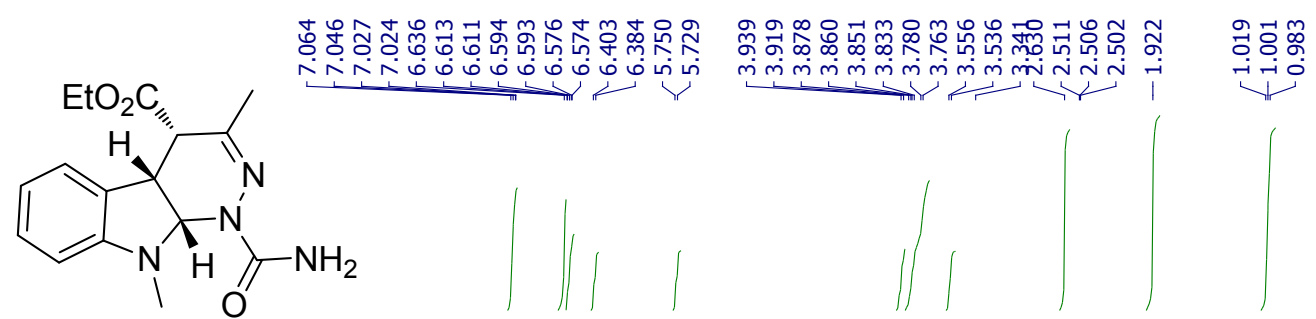

(cis, cis)-3z

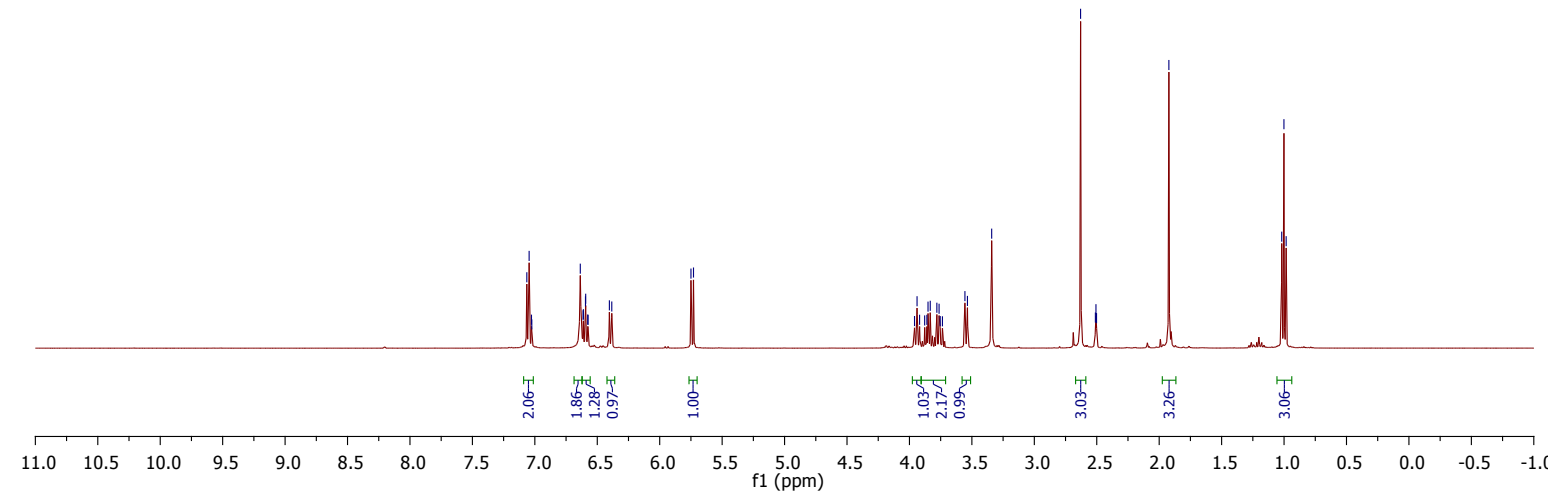

${ }^{13} \mathrm{C}$ NMR of $(c i s, c i s)-3 z$ (100 MHz, DMSO- $\left.d_{6}\right)$

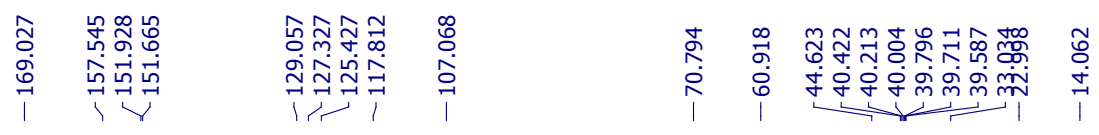

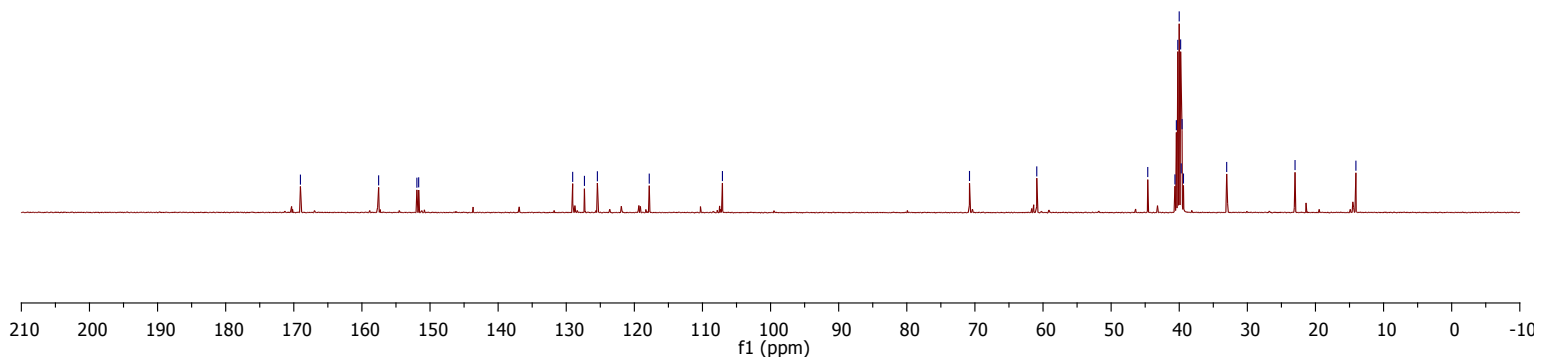


NOESY NMR of (cis,cis)-3z (400 MHz, DMSO- $\left.d_{6}\right)$

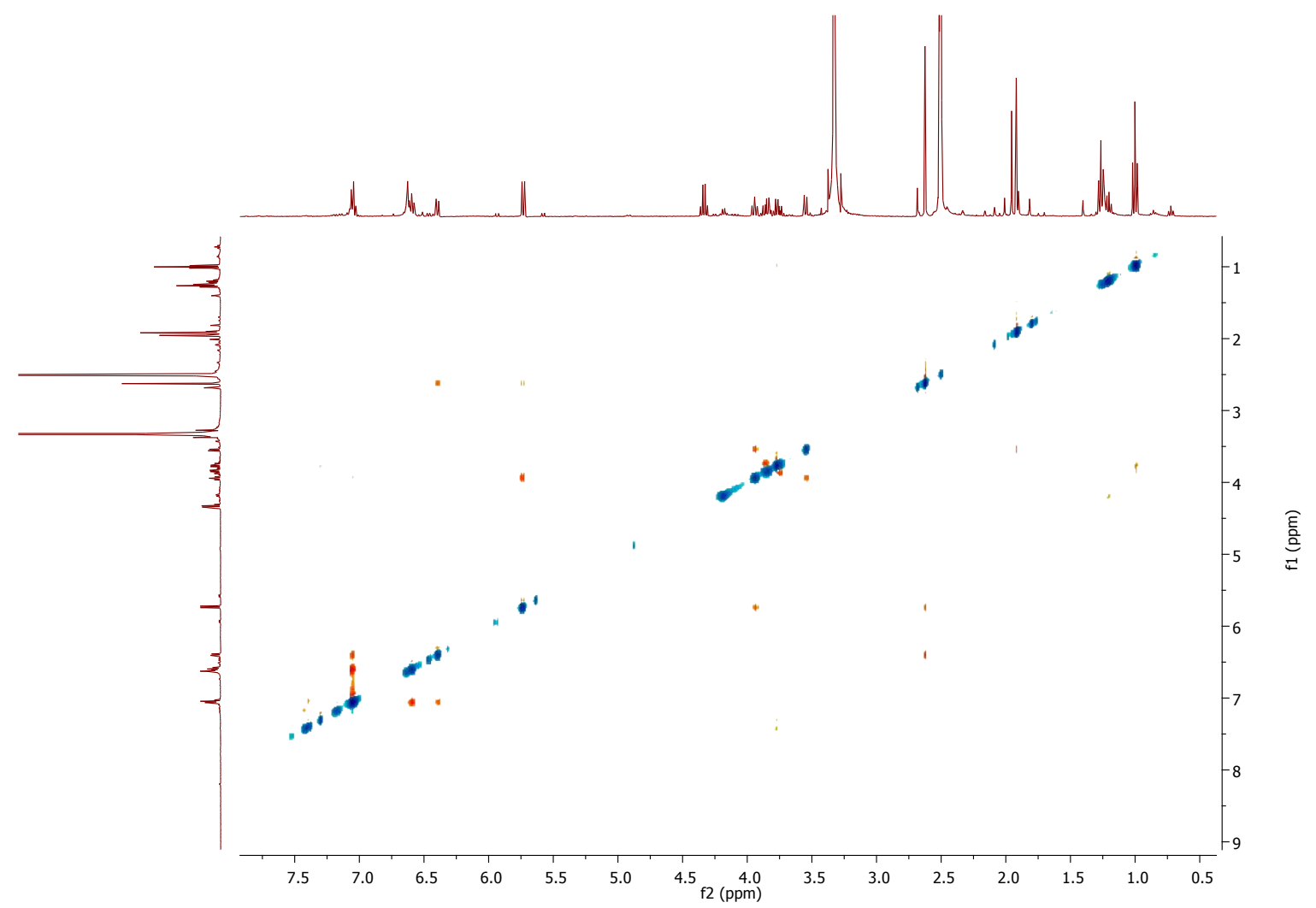


${ }^{1}$ H NMR of (cis,trans)-3z (400 MHz, DMSO-d 6 )
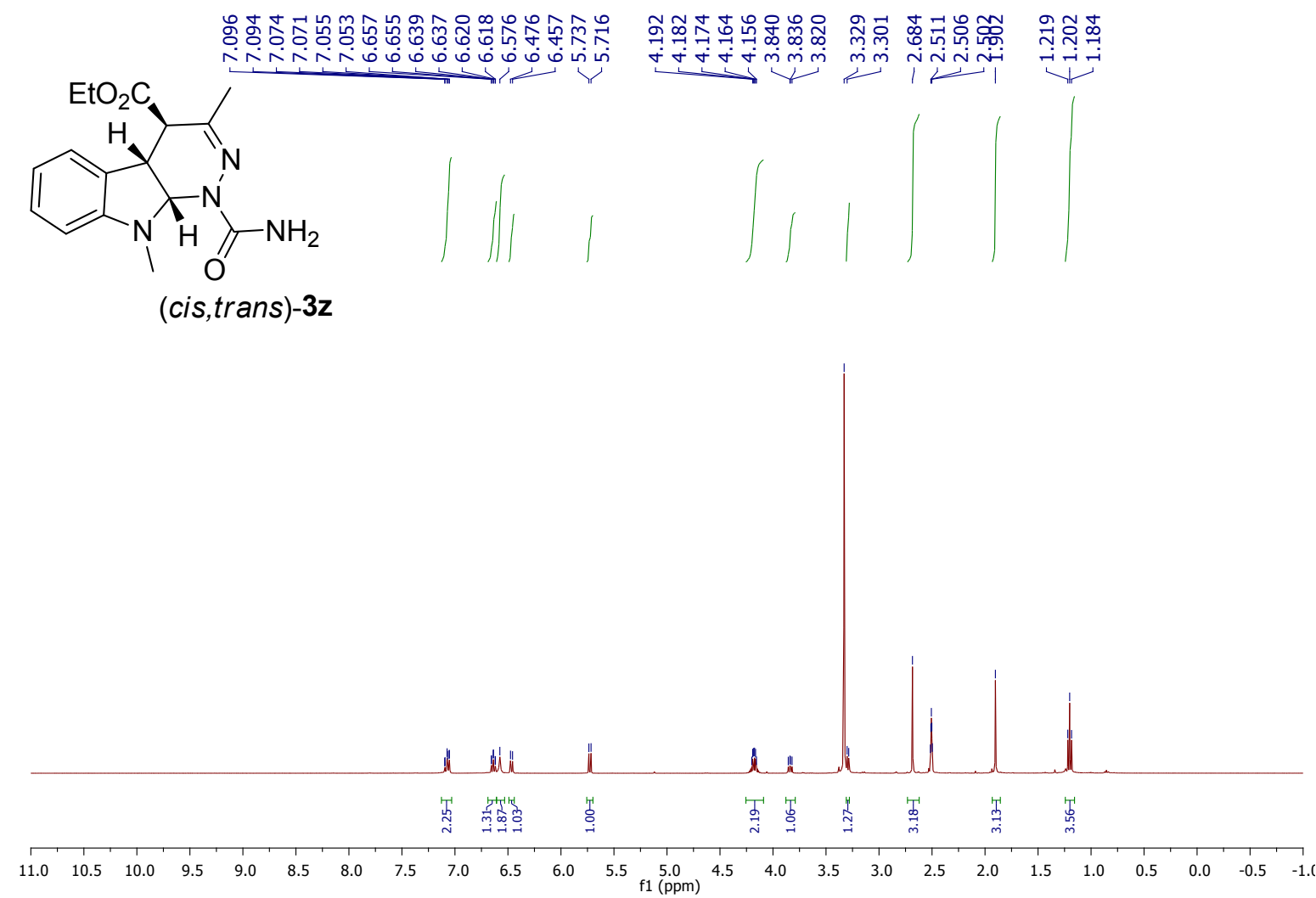

${ }^{13}$ C NMR of (cis,trans)-3z (100 MHz, DMSO- $\left.d_{6}\right)$

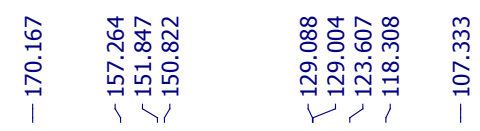

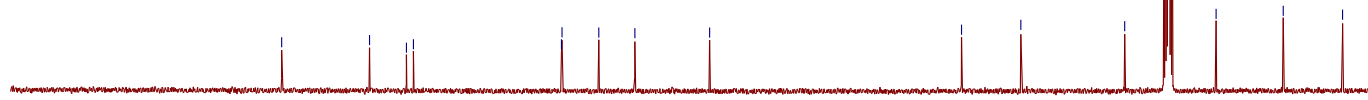


NOESY NMR of (cis,trans)-3z (400 MHz, DMSO- $d_{6}$ )

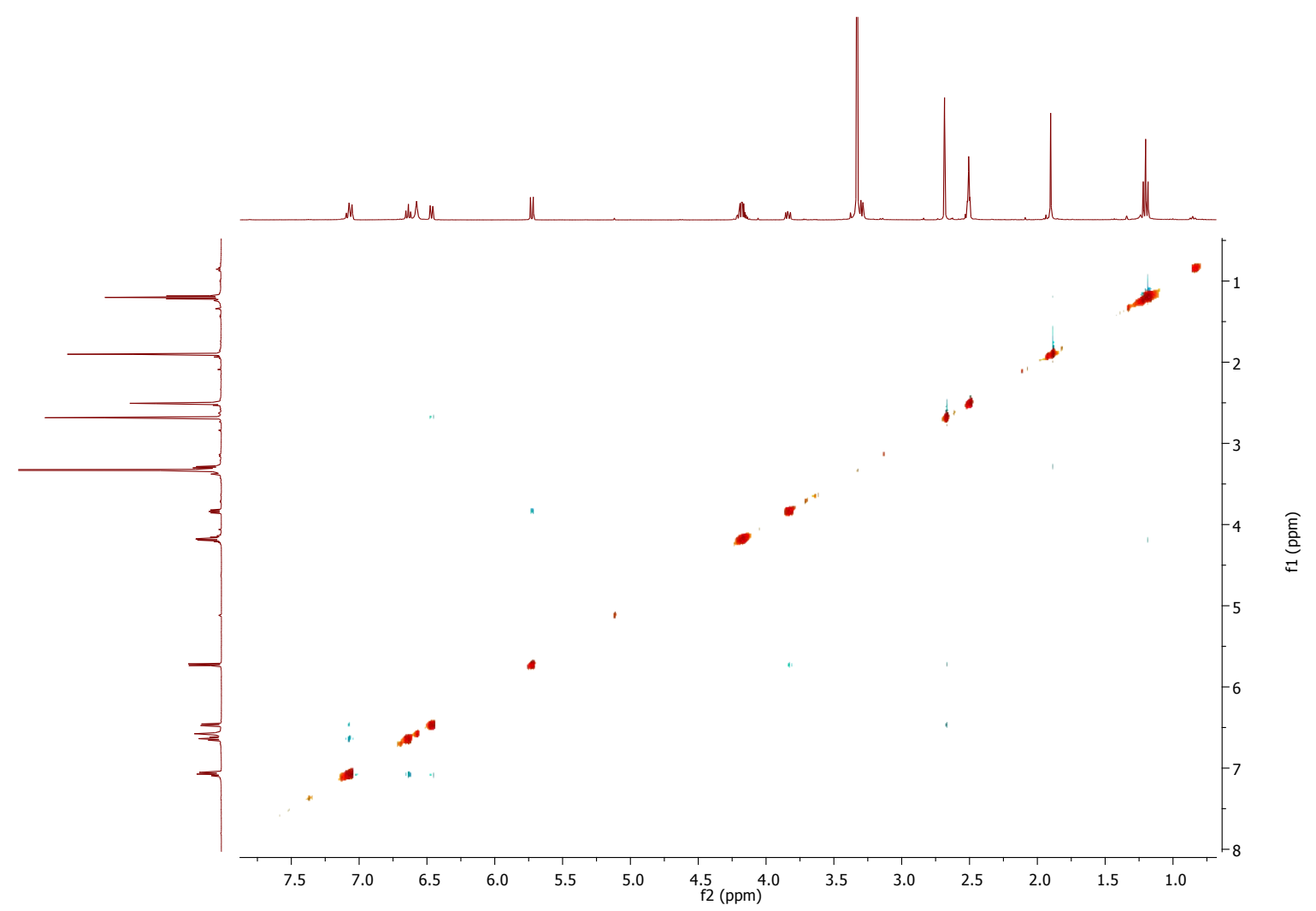


${ }^{1}$ H NMR of (cis,cis)-3ab (400 MHz, DMSO-d $\left.d_{6}\right)$

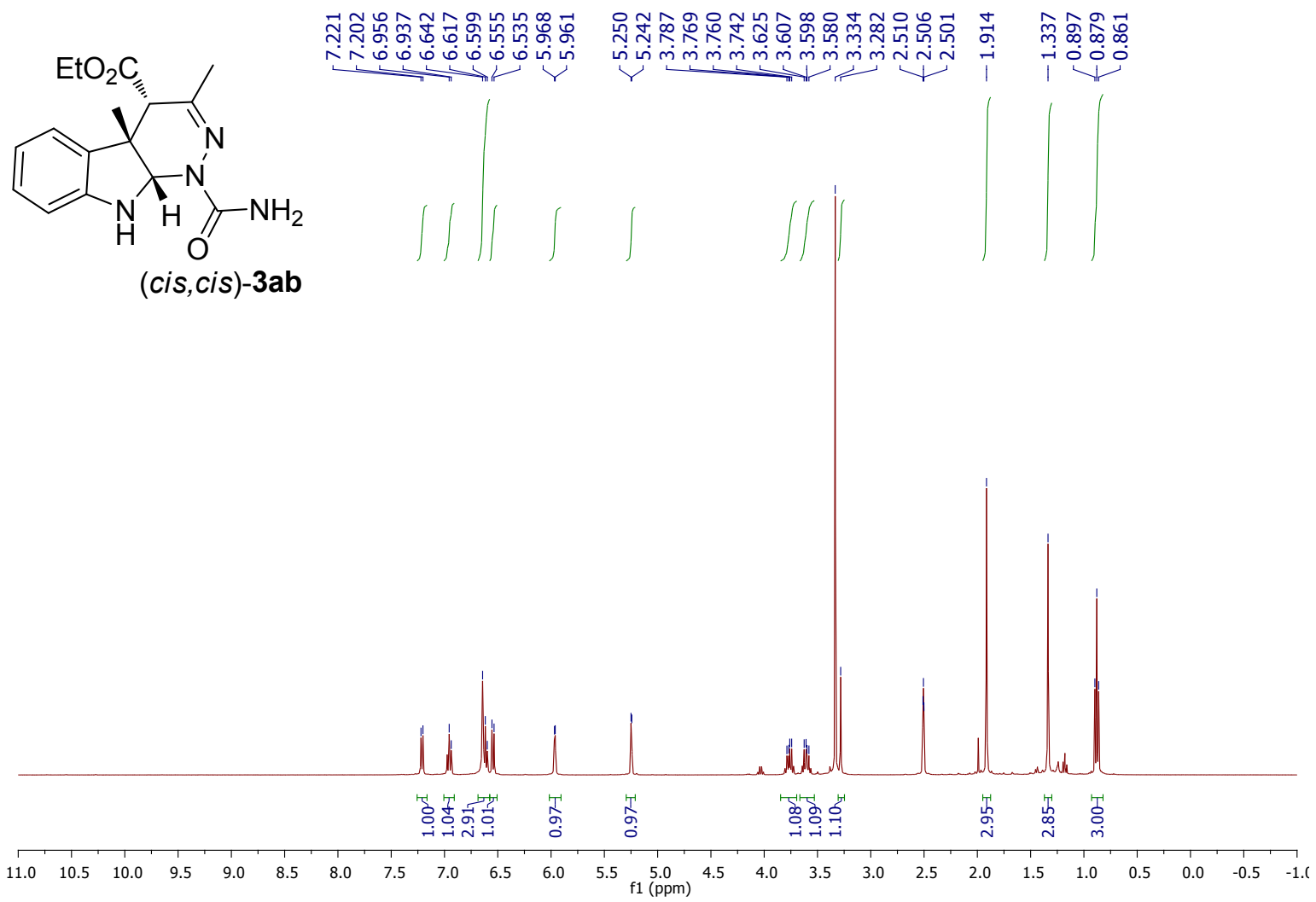

${ }^{13}$ C NMR of (cis,cis)-3ab (100 MHz, DMSO-d 6 )

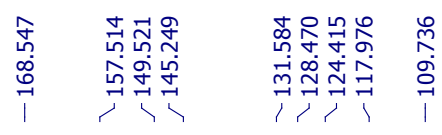

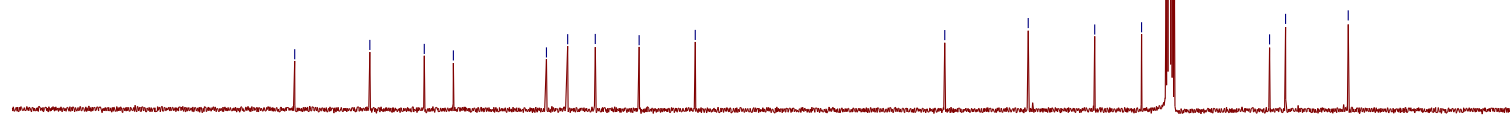

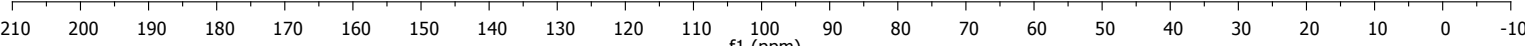


Supporting Information: S38

NOESY NMR of (cis,cis)-3ab (400 MHz, DMSO-d $\left.d_{6}\right)$

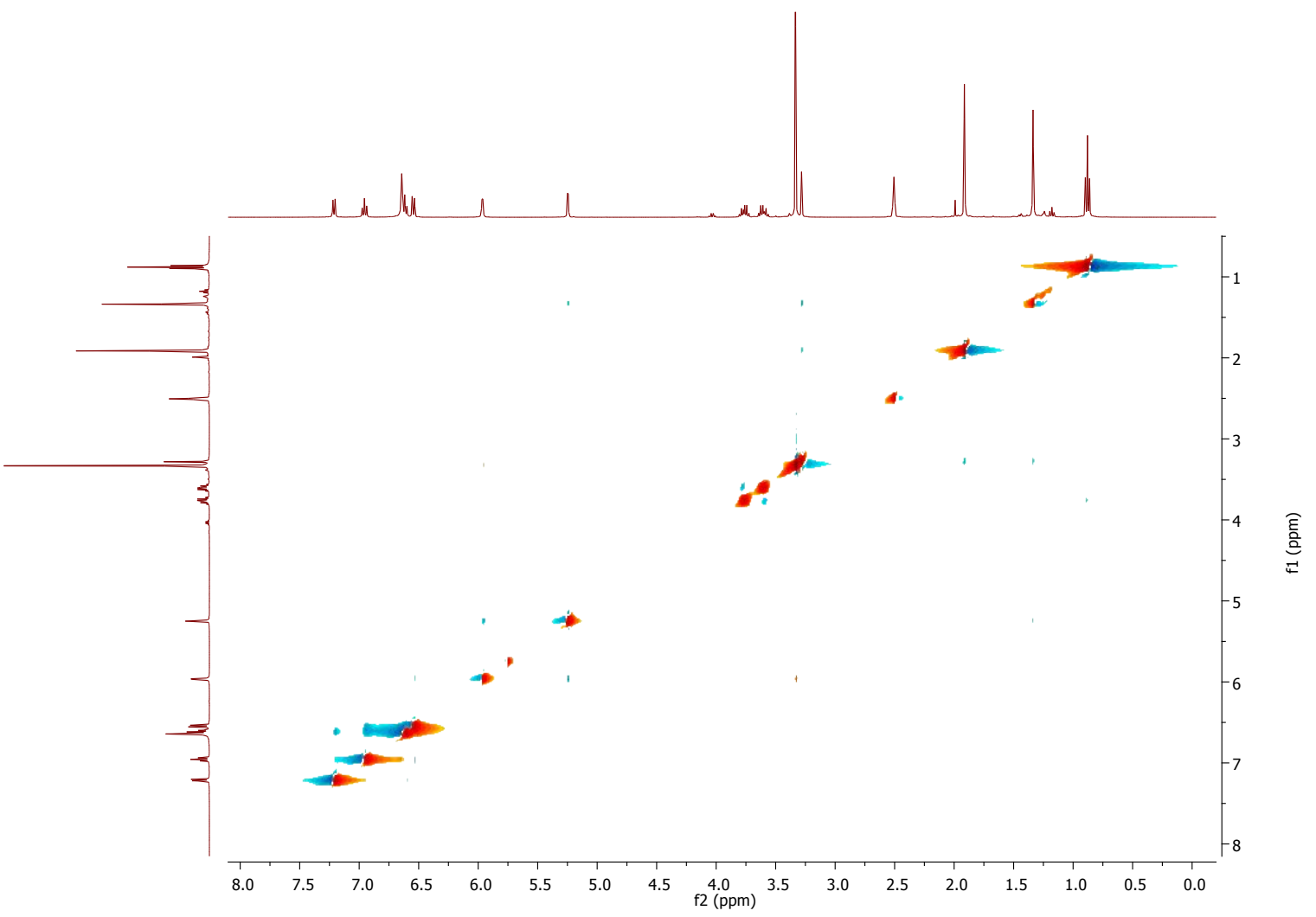

38 
${ }^{1}$ H NMR of 3ad (400 MHz, DMSO- $d_{6}$ )
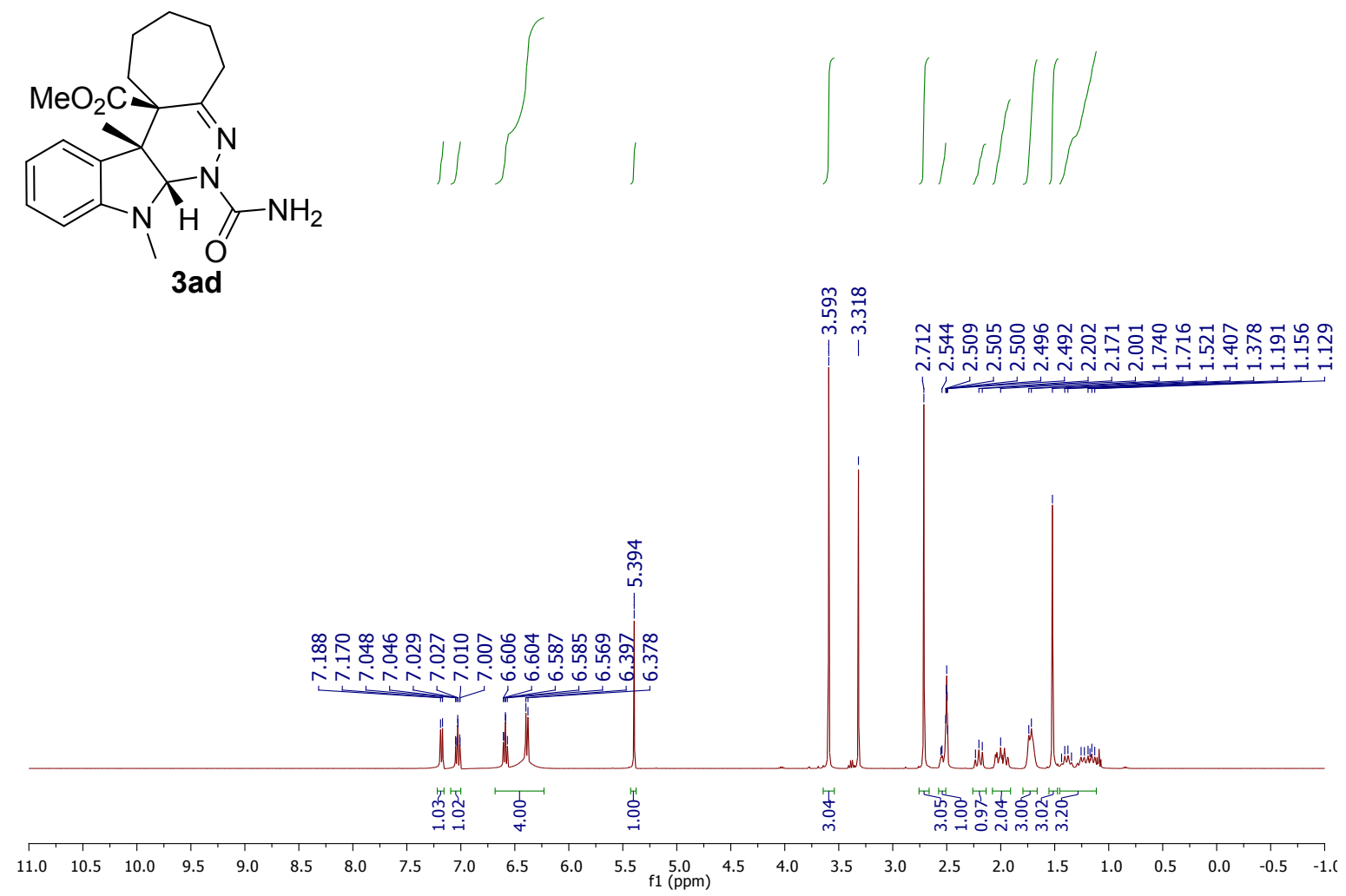

${ }^{13} \mathrm{C}$ NMR of 3ad (100 MHz, DMSO- $\left.d_{6}\right)$

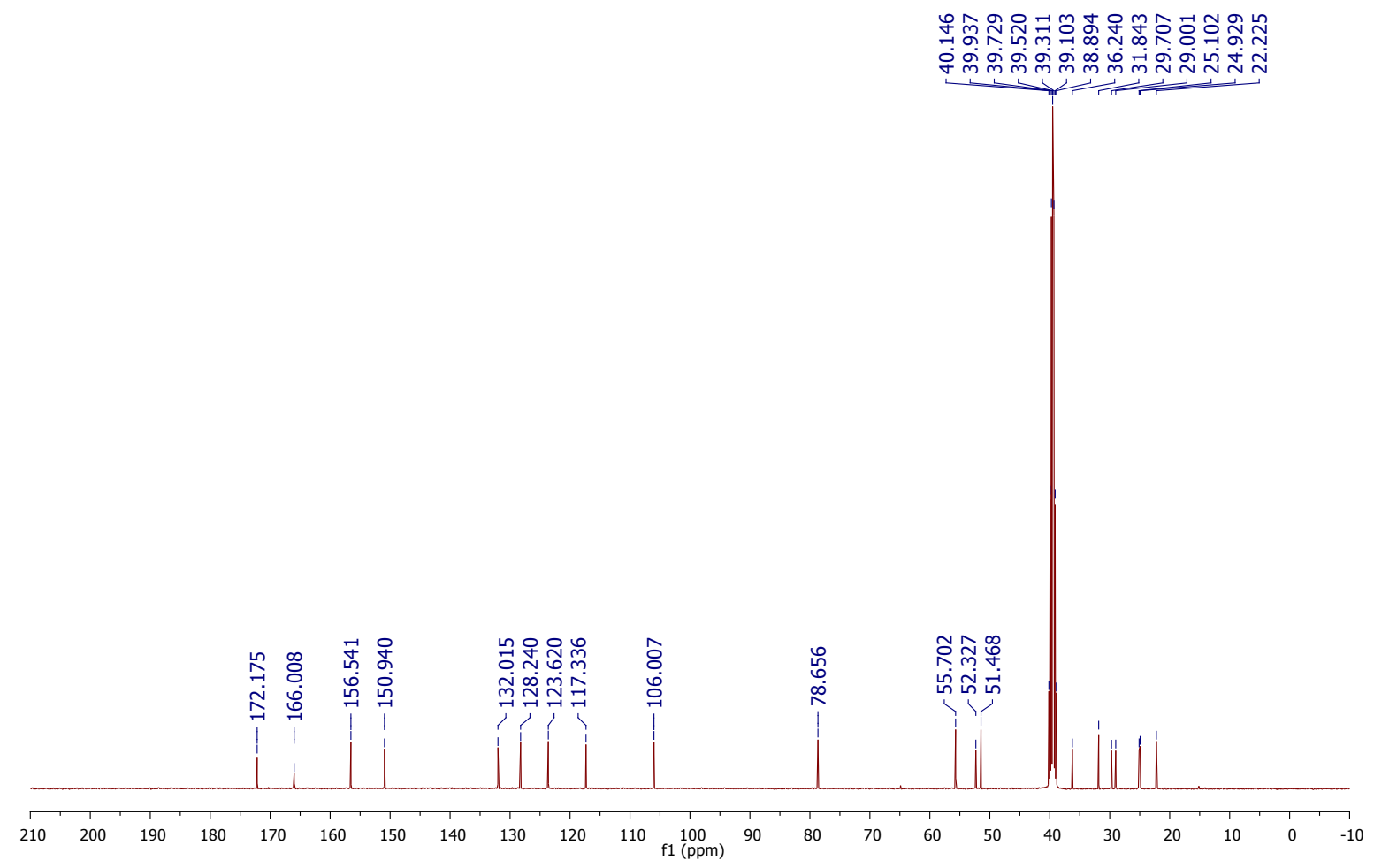


${ }^{1}$ H NMR of 3ae (400 MHz, DMSO- $\left.d_{6}\right)$

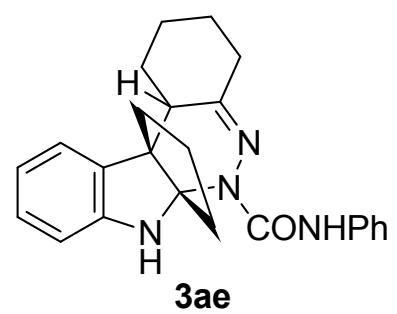

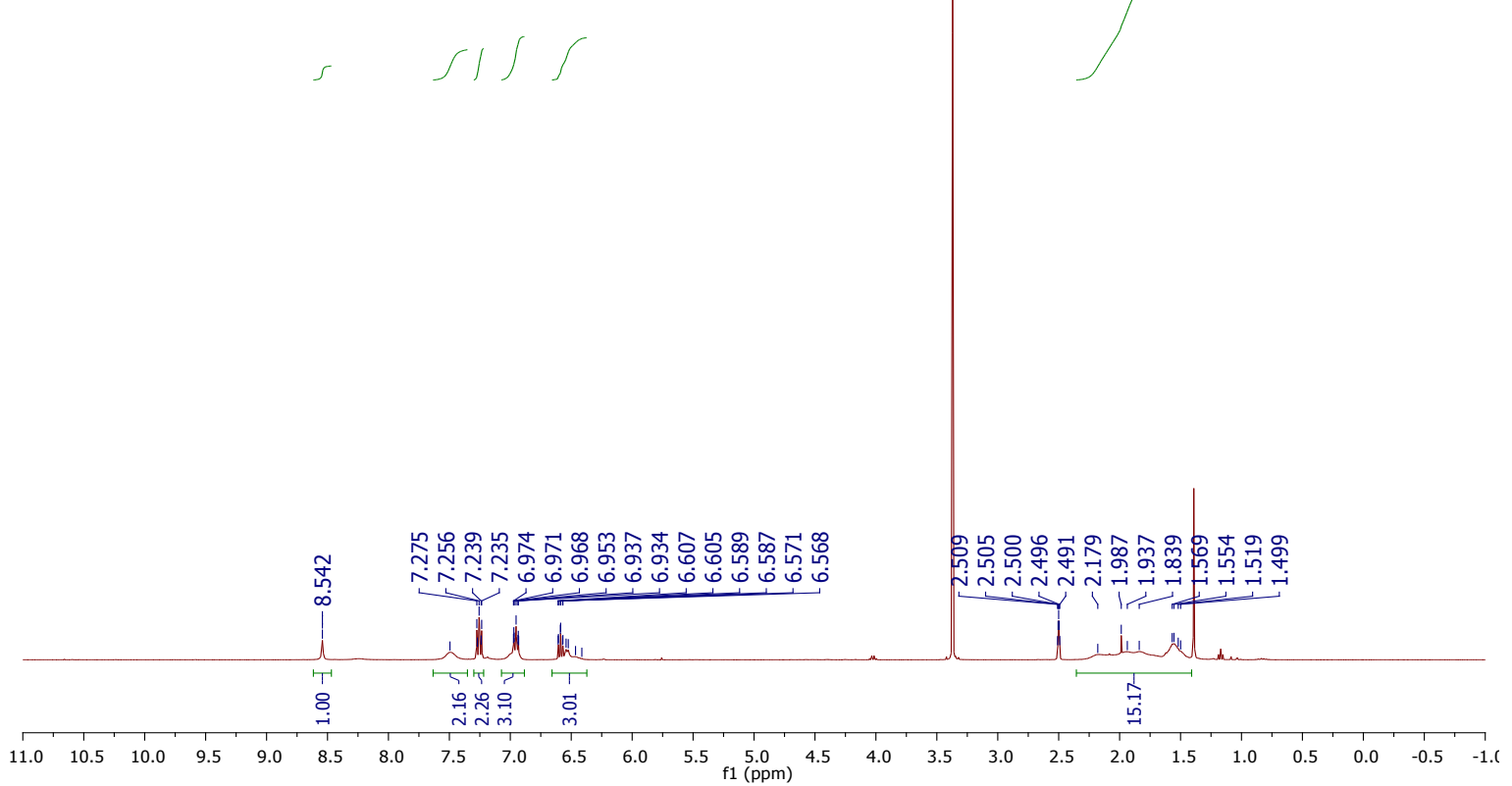

${ }^{13}$ C NMR of 3ae (100 MHz, DMSO- $d_{6}$ )

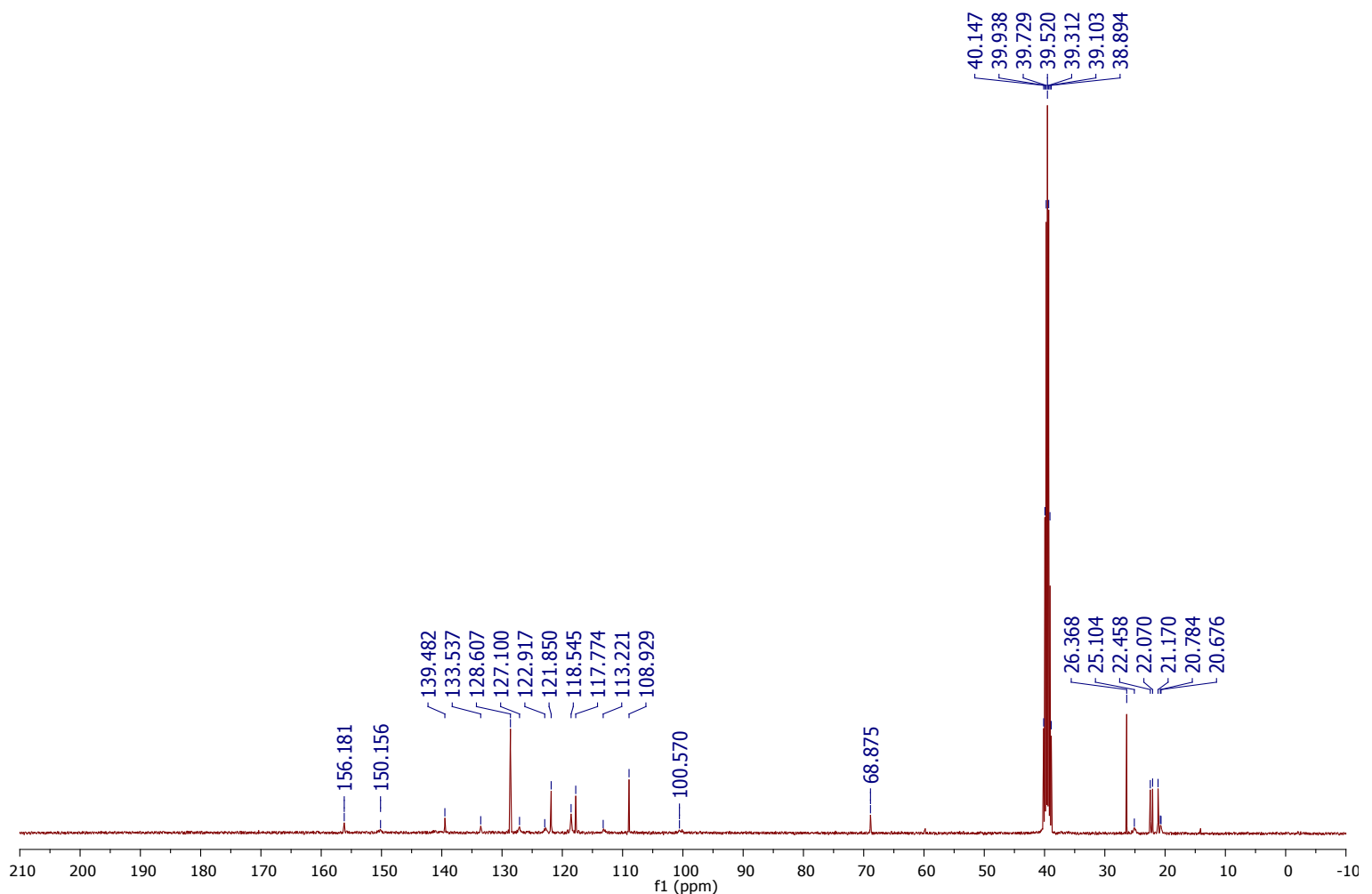


${ }^{1} \mathrm{H}$ NMR of $3 \mathrm{af}\left(400 \mathrm{MHz}, \mathrm{CDCl}_{3}\right)$<smiles>[B][C@]12Nc3ccccc3[C@]1(C)C(c1ccccc1)C(C)=NN2C(C)=O</smiles>
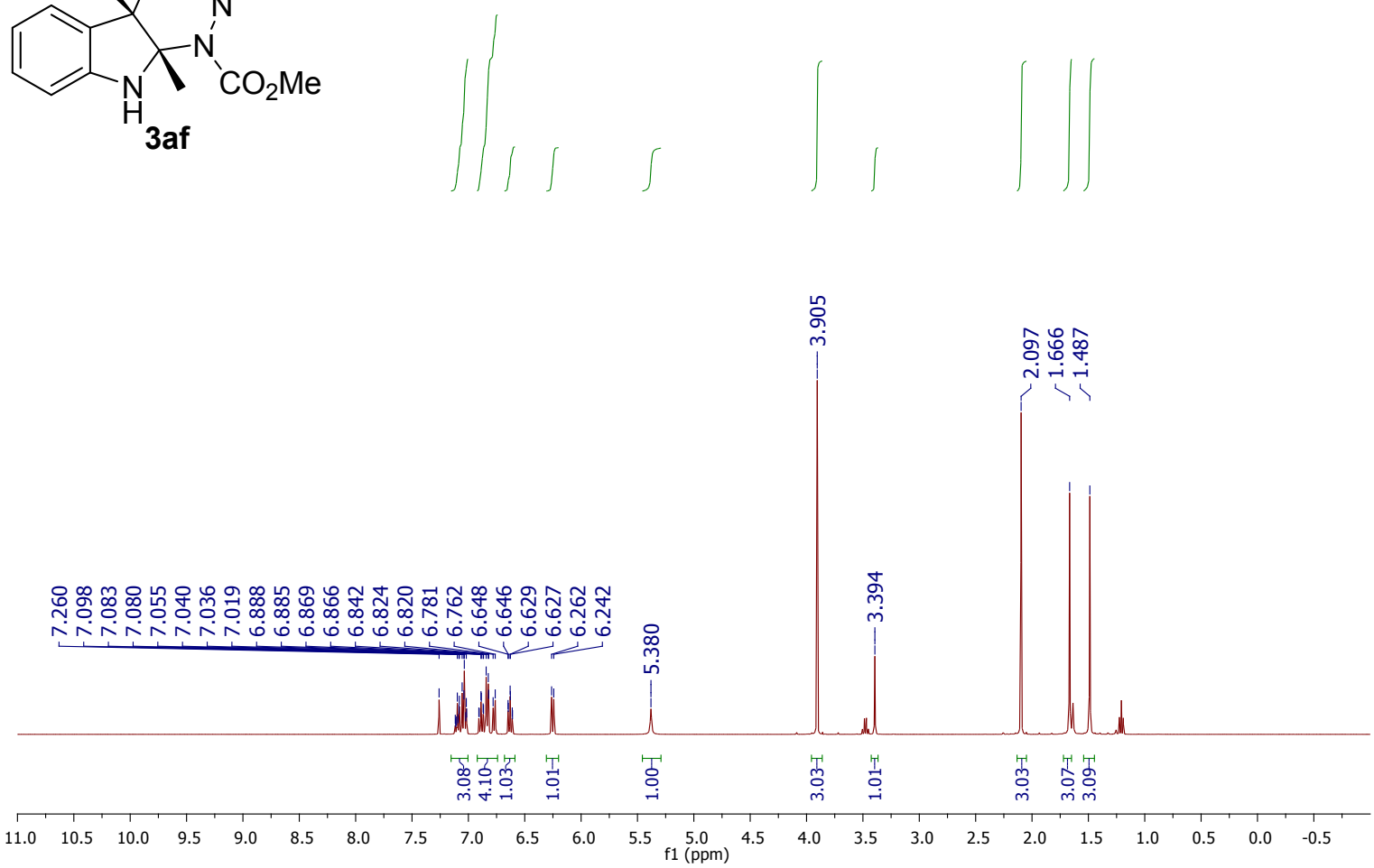

${ }^{13} \mathrm{C}$ NMR of $3 \mathrm{af}\left(100 \mathrm{MHz}, \mathrm{CDCl}_{3}\right)$

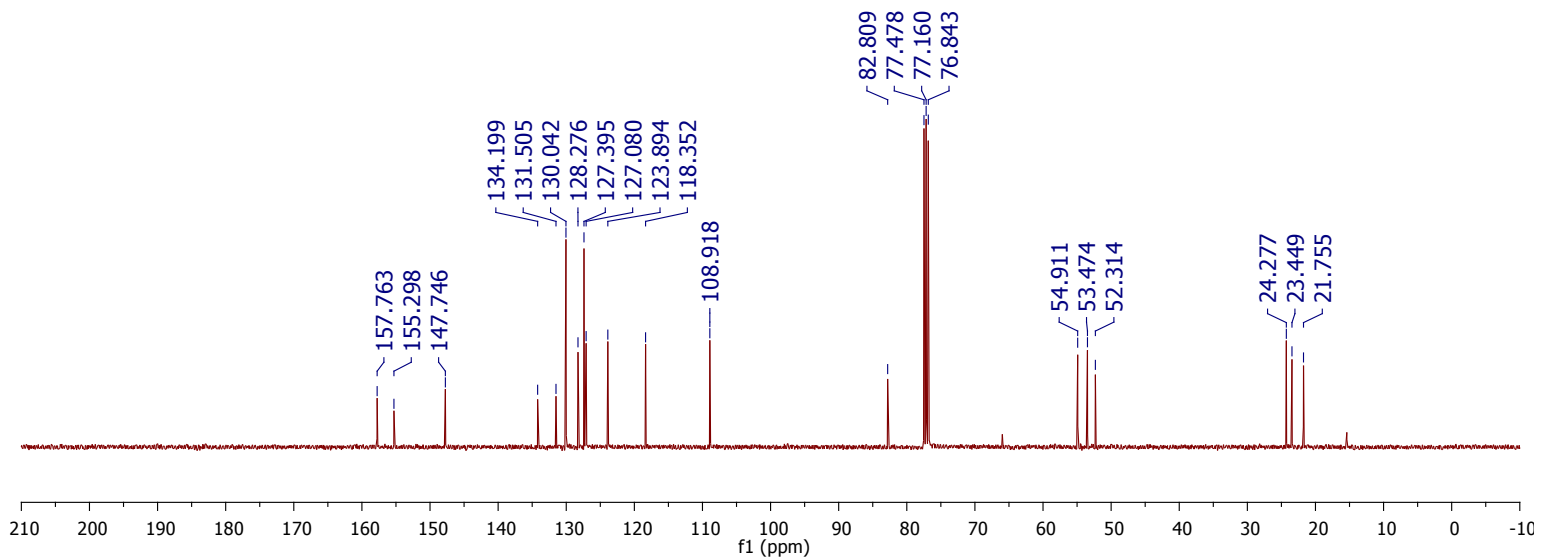


${ }^{1} \mathrm{H}$ NMR of 5a (400 MHz, DMSO- $d_{6}$ )

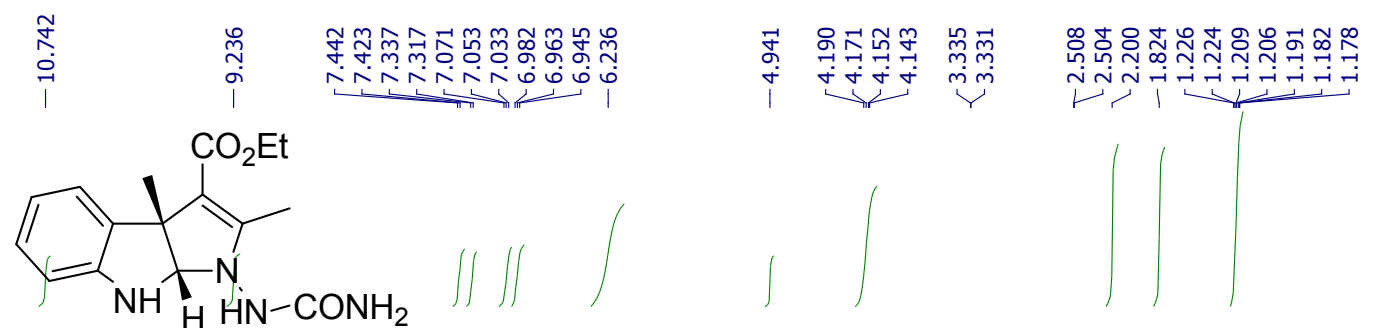

$5 a$

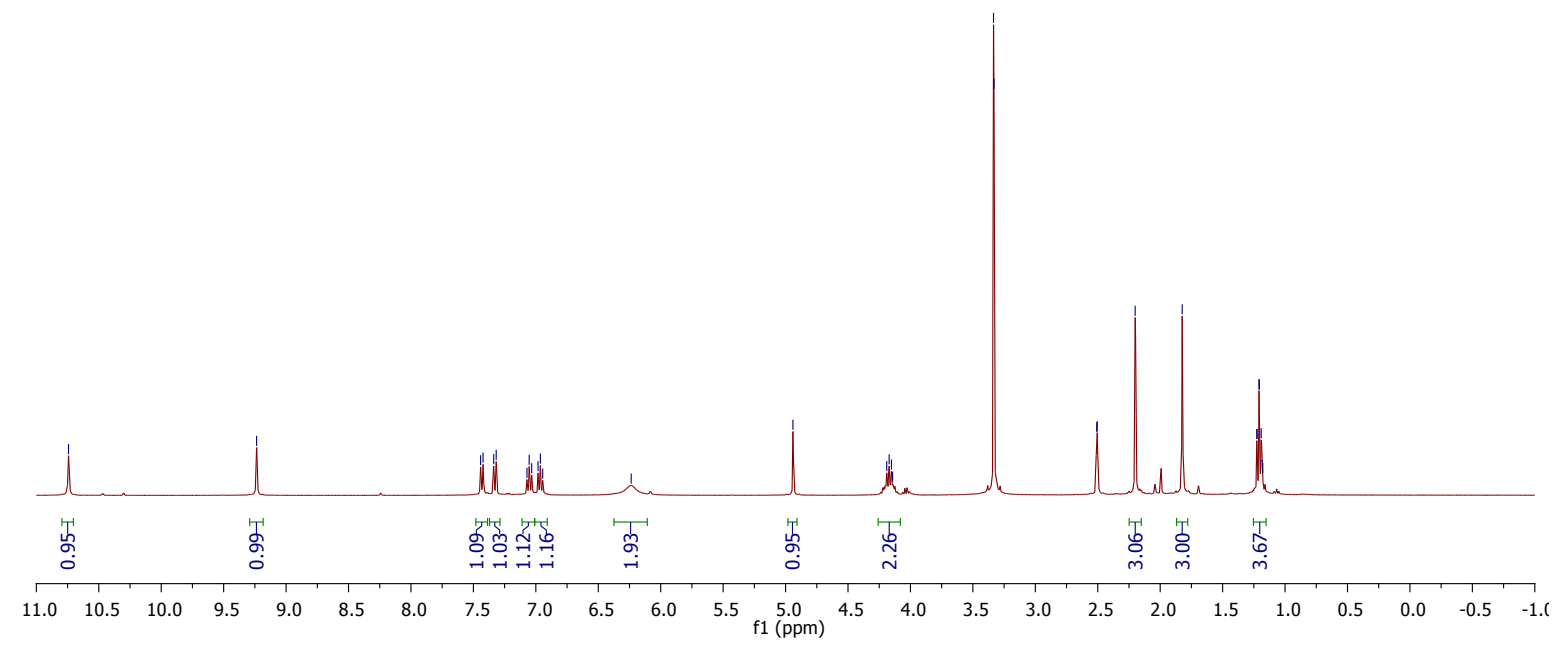

${ }^{13} \mathrm{C}$ NMR of 5a (100 MHz, DMSO- $\left.d_{6}\right)$

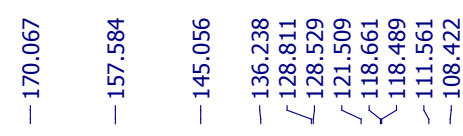

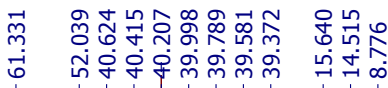

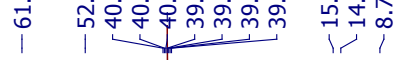

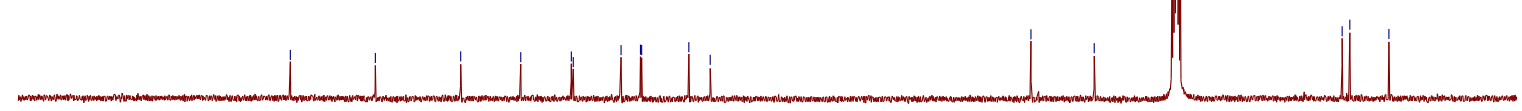

$\begin{array}{llllllllllllllllllllllllllllll}210 & 200 & 190 & 180 & 170 & 160 & 150 & 140 & 130 & 120 & 110 & 100 & 90 & 80 & 70 & 60 & 50 & 40 & 30 & 20 & 10 & 0 & -10\end{array}$ 
${ }^{1} \mathrm{H}$ NMR of $5 \mathrm{~b}\left(400 \mathrm{MHz}, \mathrm{CDCl}_{3}\right)$<smiles>CC(=O)NC1N[C@H]2N(C)c3ccccc3[C@@]2(C)C1C(C)=O</smiles>

$5 b$
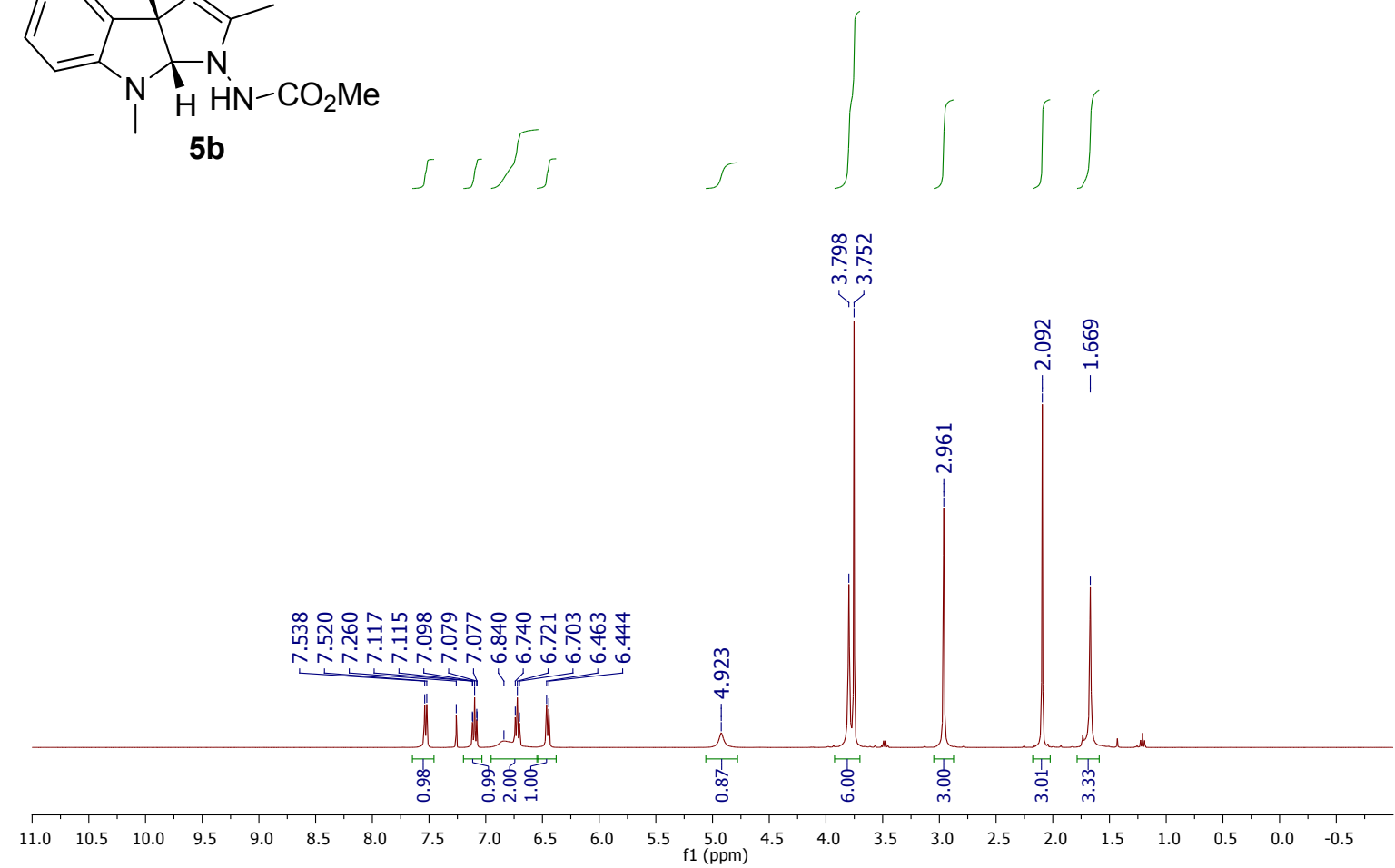

${ }^{13} \mathrm{C}$ NMR of $5 \mathrm{~b}\left(100 \mathrm{MHz}, \mathrm{CDCl}_{3}\right)$

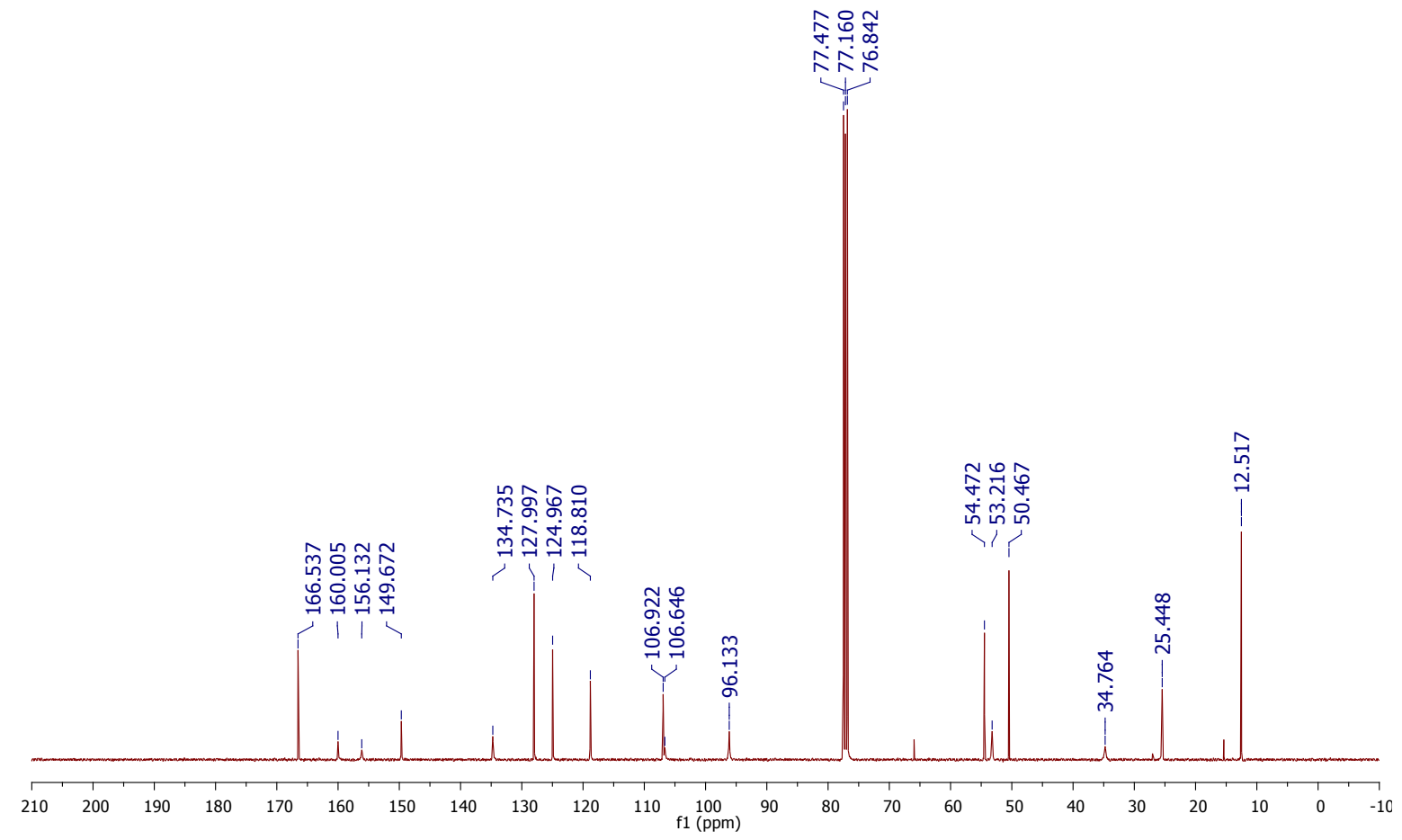


${ }^{1} \mathrm{H}$ NMR of $5 \mathrm{c}\left(400 \mathrm{MHz}\right.$, DMSO- $\left.d_{6}\right)$<smiles>CC(=O)NC1=C(C)[C@@]2(C)c3ccccc3N[C@]2(C)N1</smiles>

$5 c$

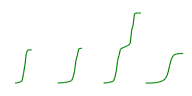

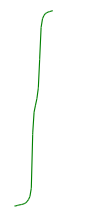
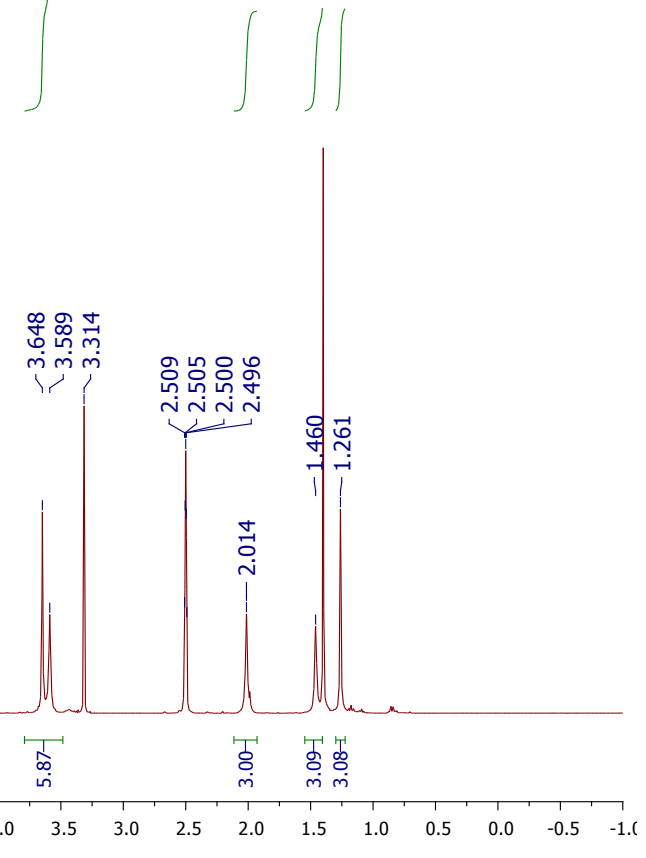

${ }^{13} \mathrm{C}$ NMR of $5 \mathrm{c}\left(100 \mathrm{MHz}, \mathrm{DMSO}-d_{6}\right)$

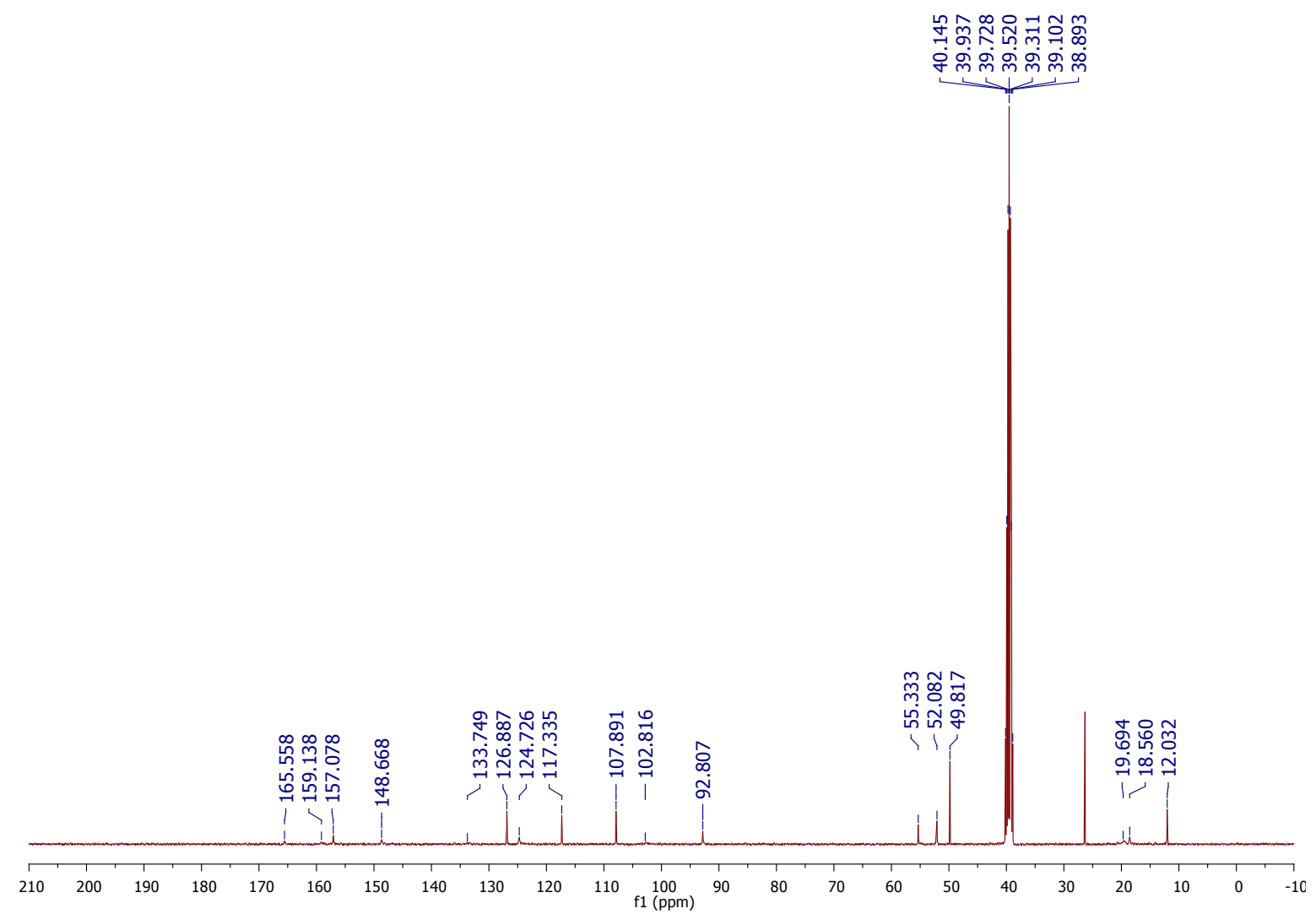




\section{HMQC NMR of 5c (400 MHz, DMSO- $\left.d_{6}\right)$}

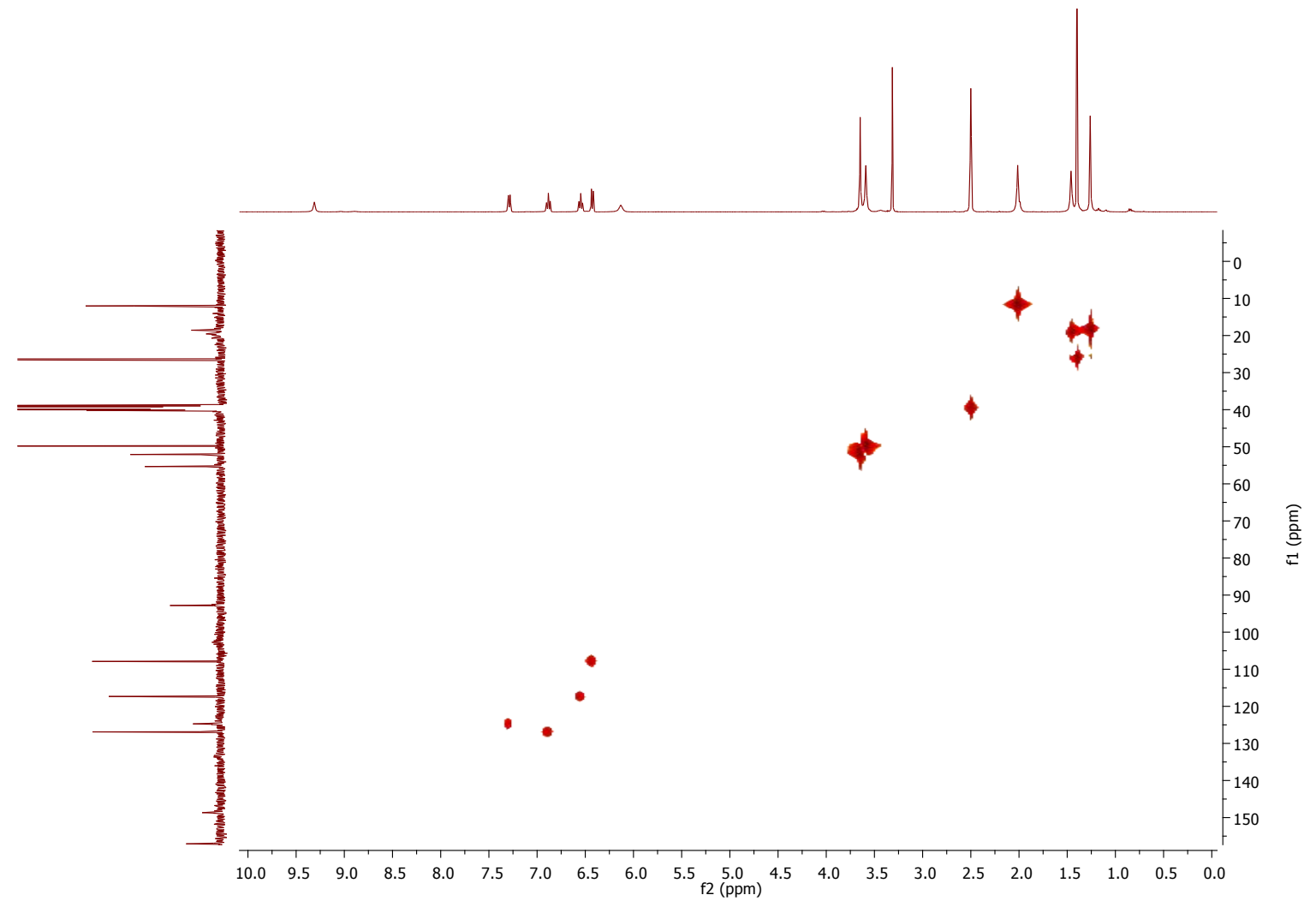

HMBC NMR of $5 \mathrm{c}\left(400 \mathrm{MHz}\right.$, DMSO- $\left.d_{6}\right)$

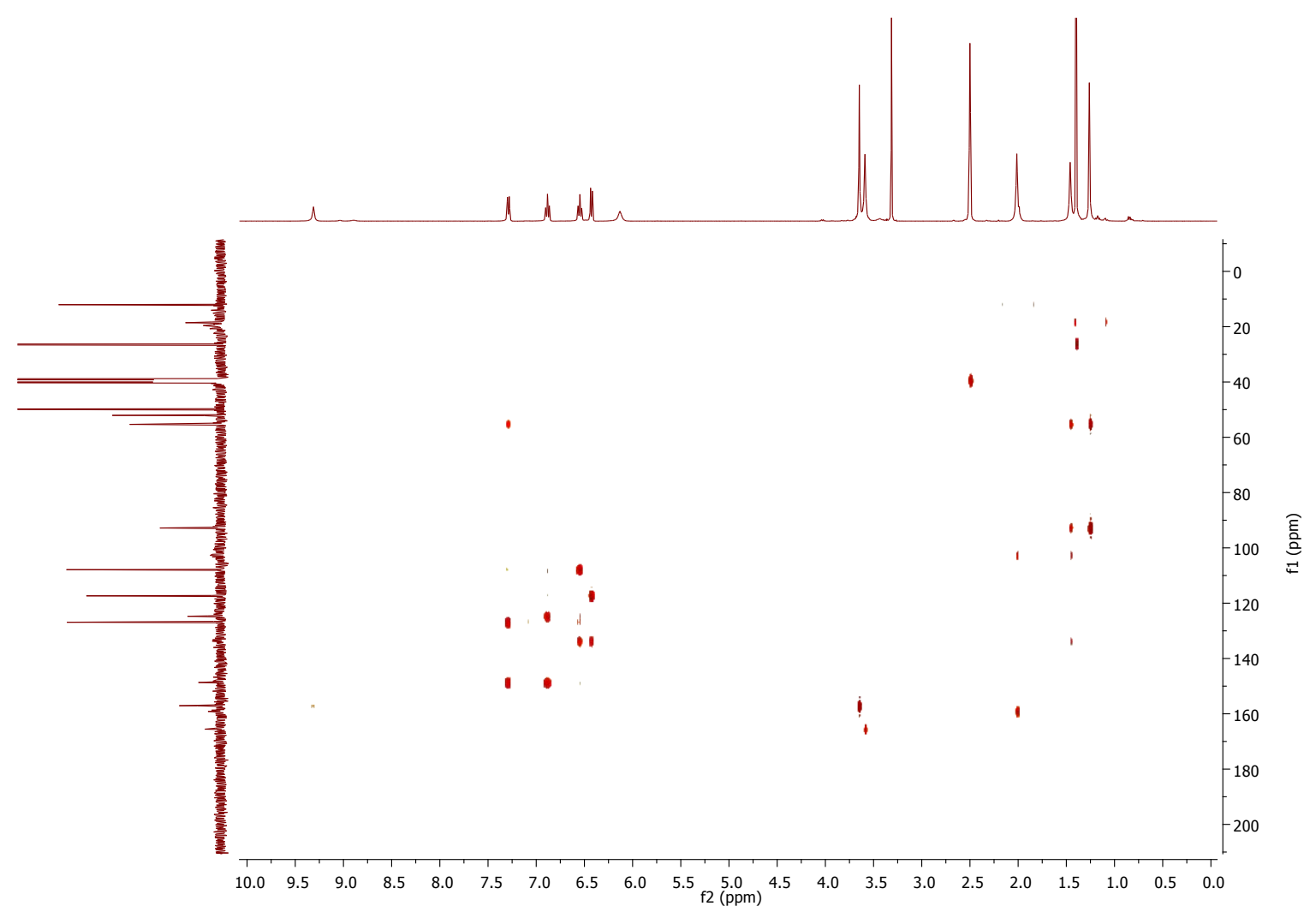


${ }^{1} \mathrm{H}$ NMR of $5 \mathrm{~d}\left(400 \mathrm{MHz}\right.$, DMSO- $\left.d_{6}\right)$<smiles>CC(=O)OC(=O)N1C(C)=C(C(=O)OCc2ccccc2)[C@]2(C)c3ccccc3N[C@]12C</smiles>

$5 d$

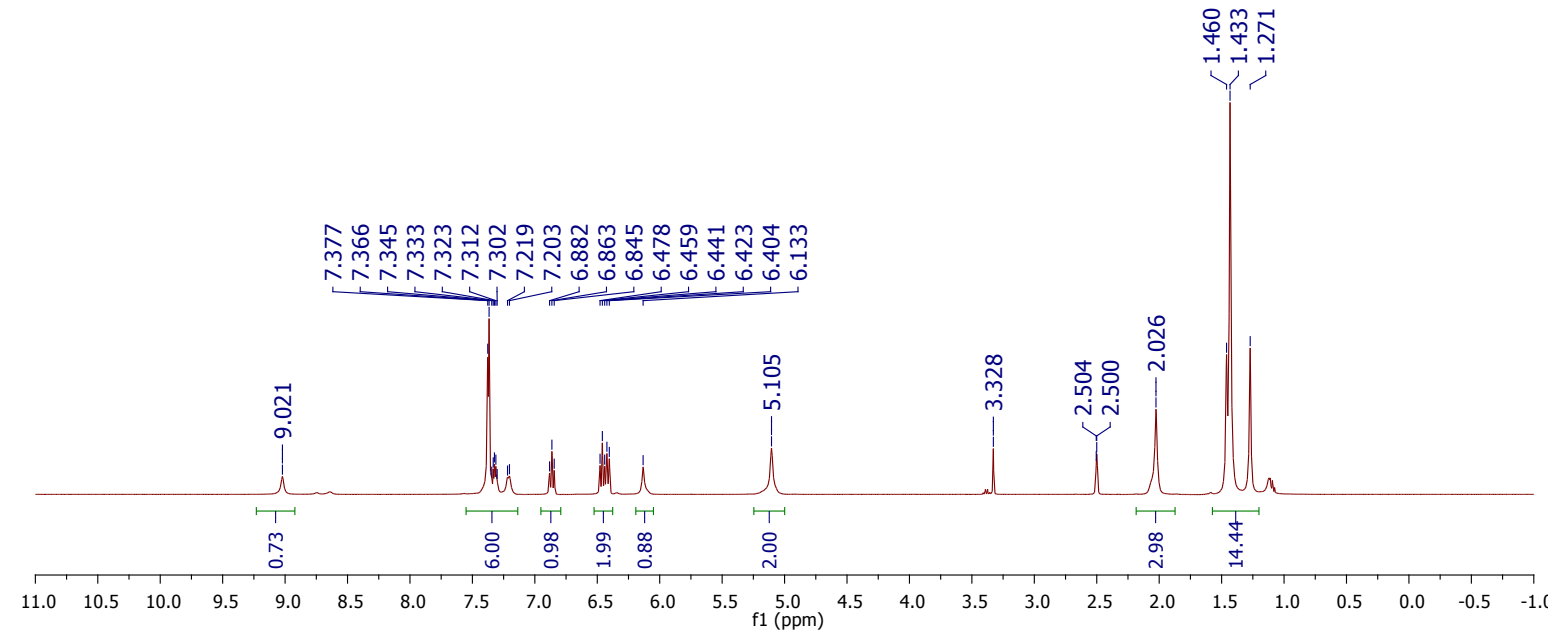

${ }^{13} \mathrm{C}$ NMR of $5 \mathrm{~d}\left(100 \mathrm{MHz}, \mathrm{DMSO}-d_{6}\right)$

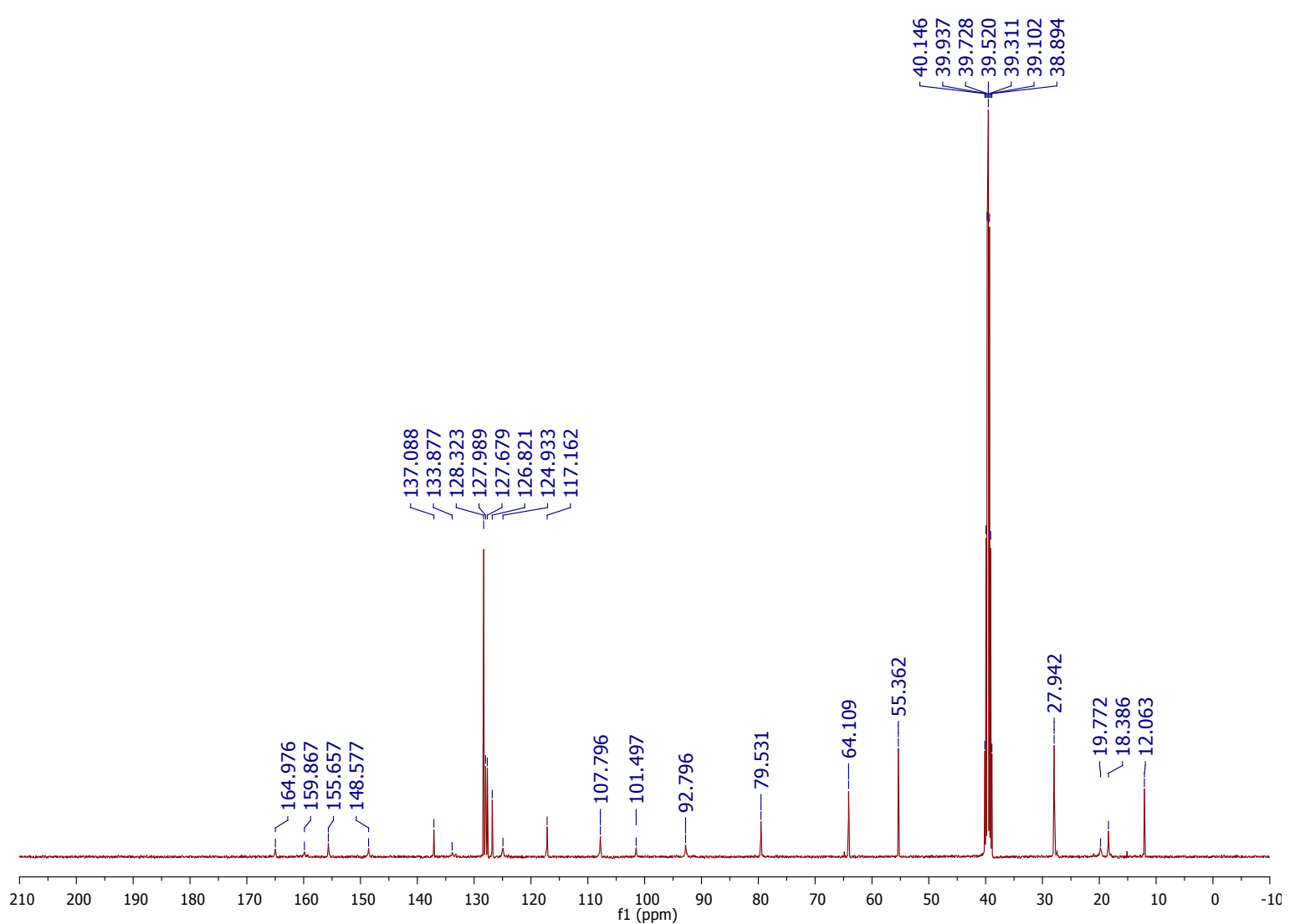


${ }^{1} \mathrm{H}$ NMR of $5 \mathrm{e}$ (400 MHz, DMSO- $\left.d_{6}\right)$<smiles>CCOC(=O)C1=C(C)N[C@]2(C)[C@H](C)c3ccccc3N[C@]12C</smiles>

$5 e$

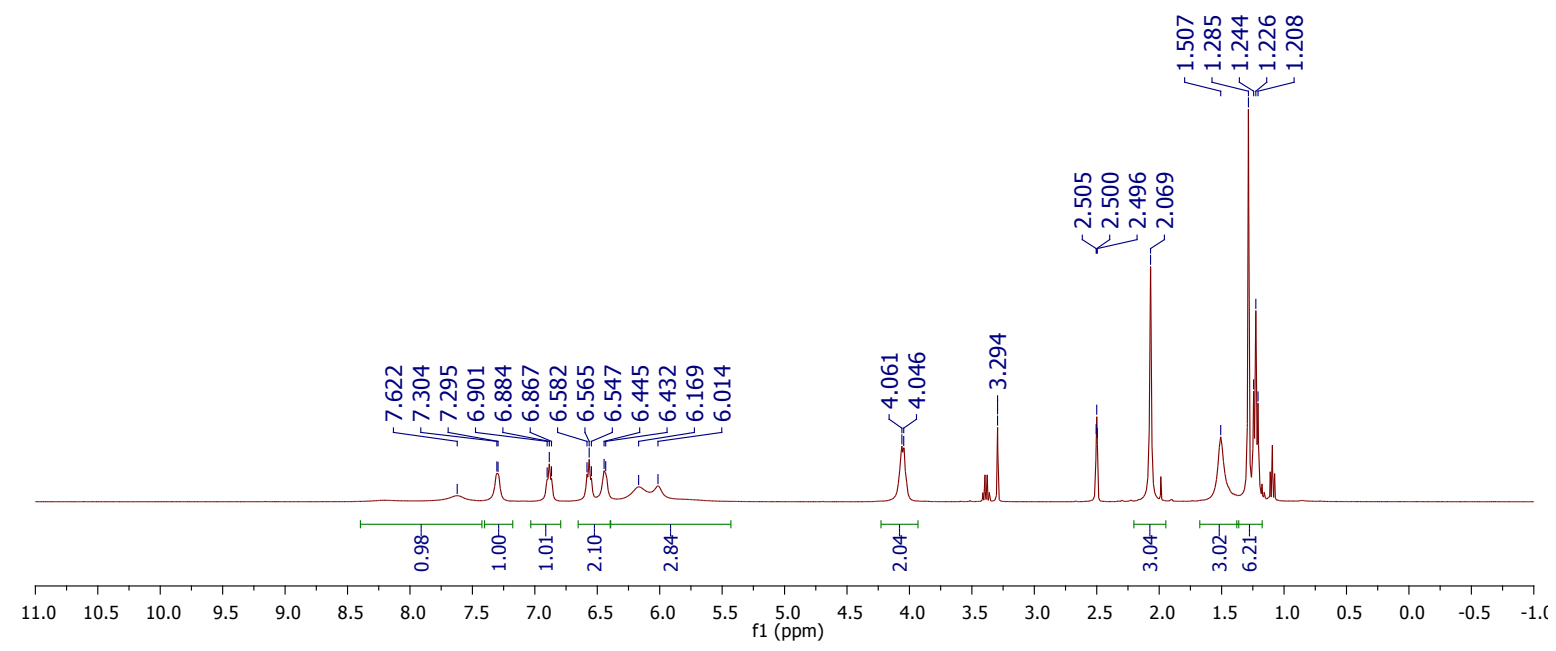

${ }^{13} \mathrm{C}$ NMR of $5 e$ (100 MHz, DMSO- $\left.d_{6}\right)$

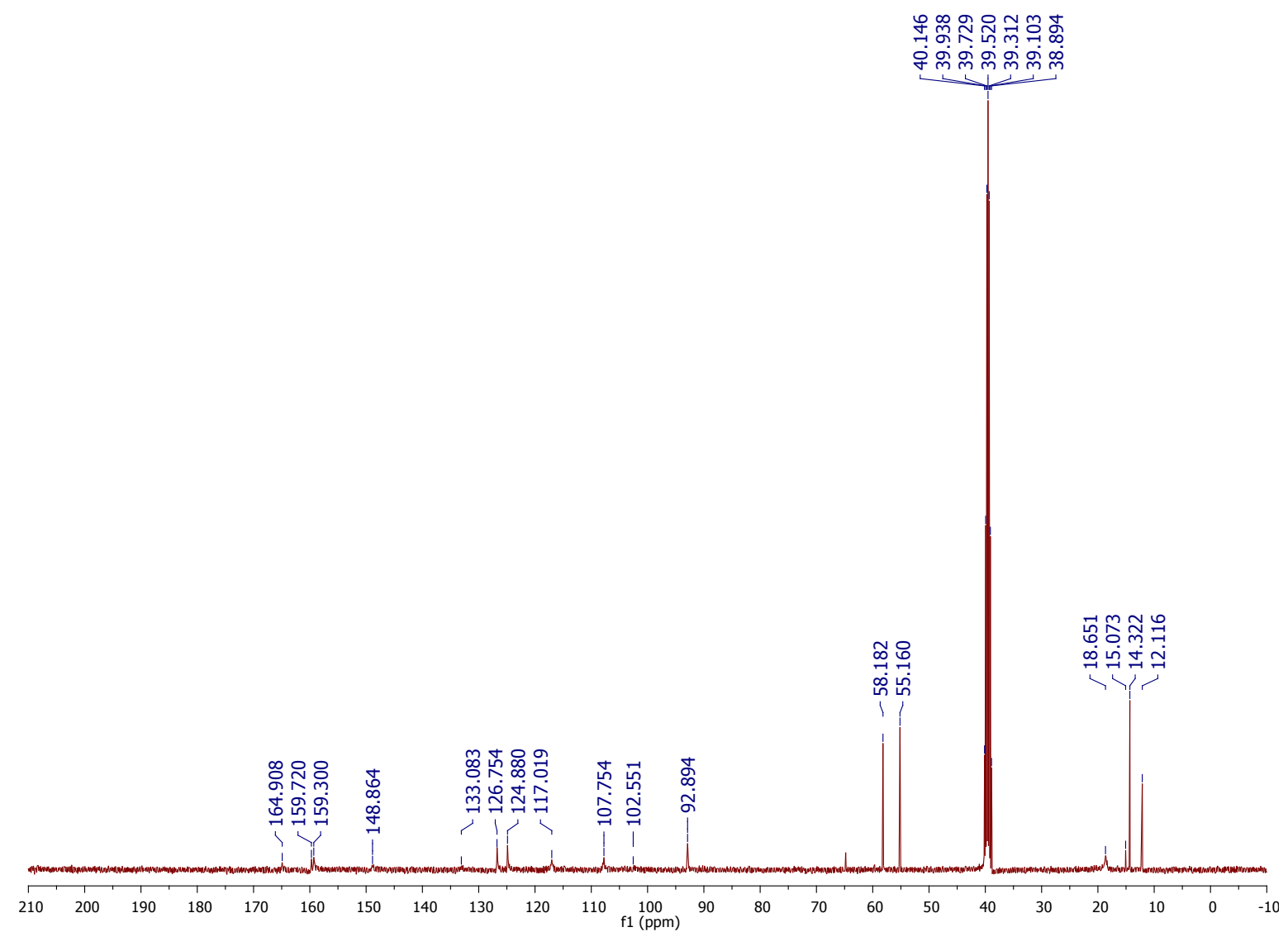


${ }^{1} \mathrm{H}$ NMR of $5 \mathrm{f}$ (400 MHz, DMSO- $\left.d_{6}\right)$
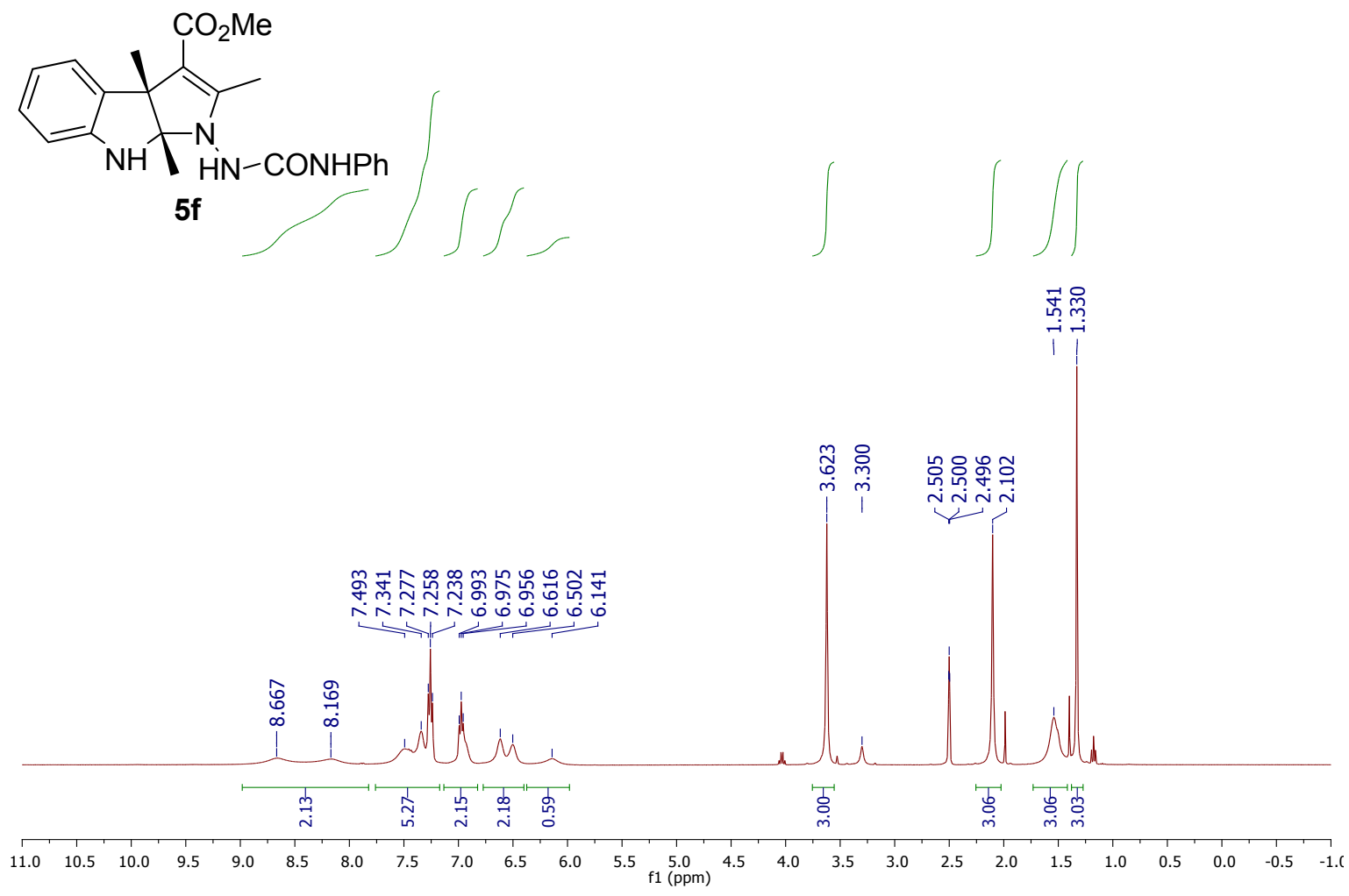

${ }^{13} \mathrm{C}$ NMR of $5 \mathrm{f}\left(100 \mathrm{MHz}, \mathrm{DMSO}-d_{6}\right)$

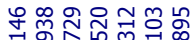

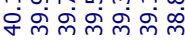
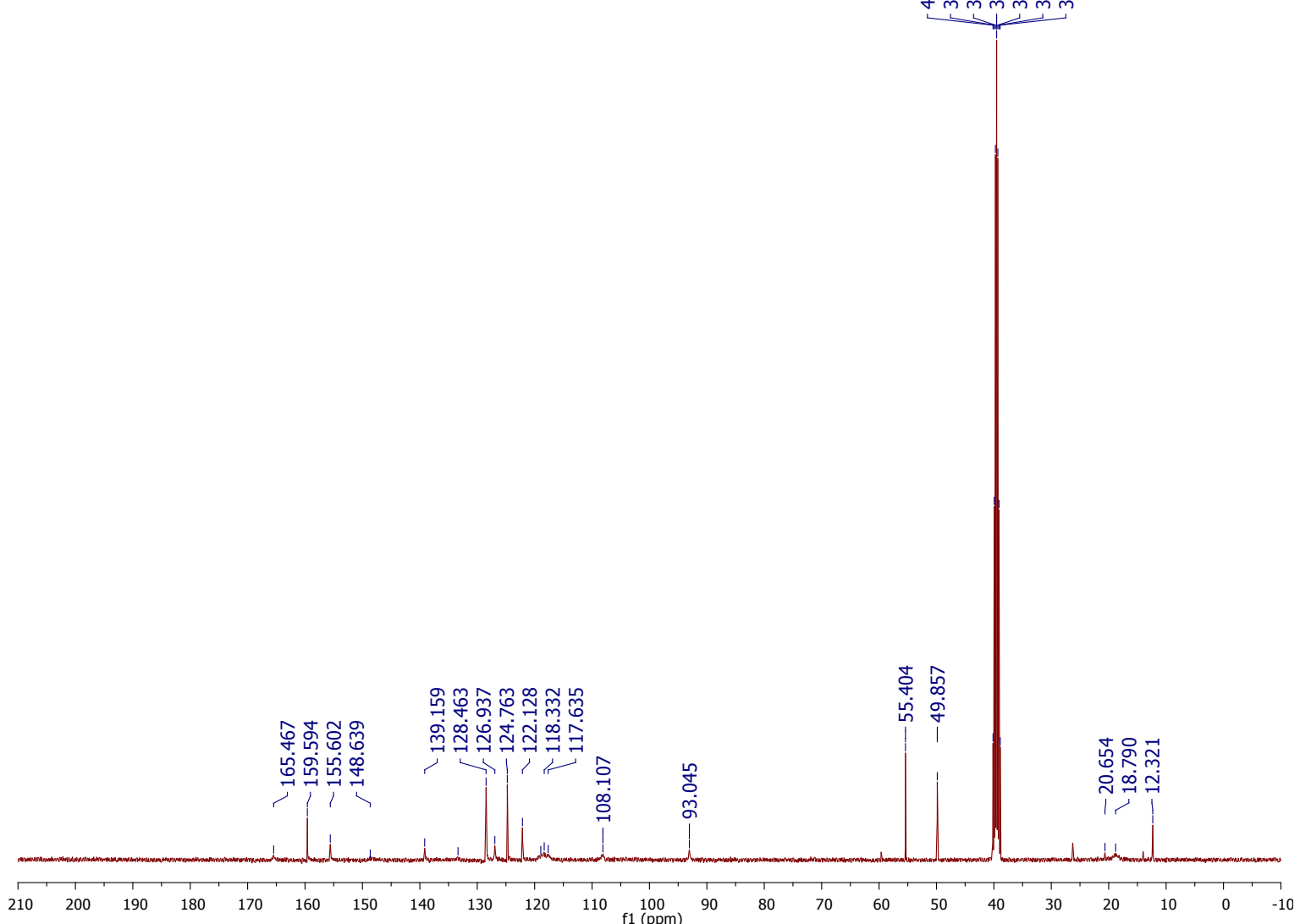
${ }^{1} \mathrm{H}$ NMR of $5 \mathrm{~g}\left(400 \mathrm{MHz}, \mathrm{CDCl}_{3}\right)$<smiles>CCC1=C(C(C)=O)[C@@]2(C)c3ccccc3NC2(C)N1</smiles>

$5 g$
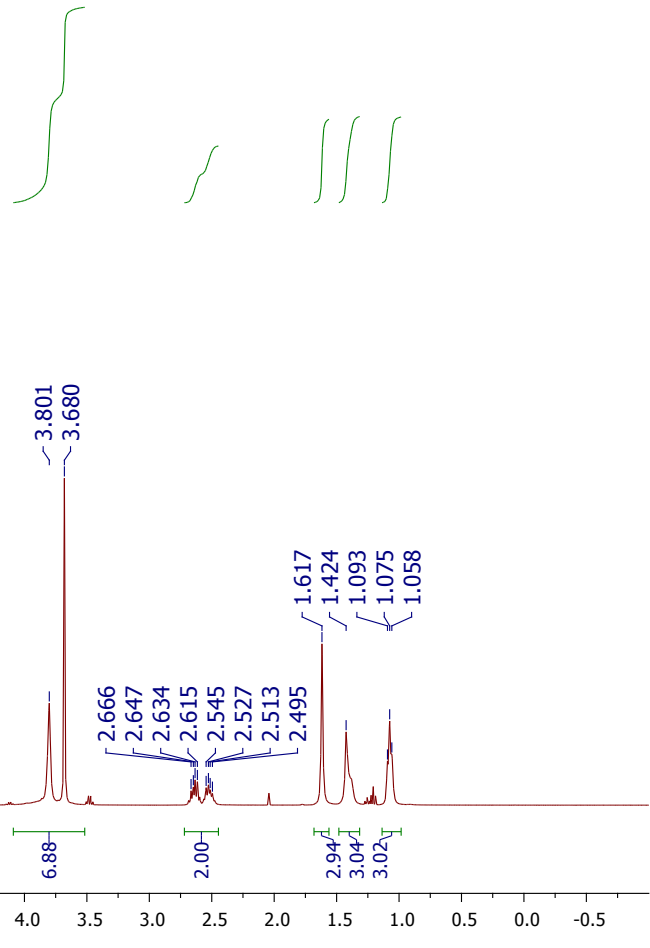

${ }^{13} \mathrm{C}$ NMR of $5 \mathrm{~g}\left(100 \mathrm{MHz}, \mathrm{CDCl}_{3}\right)$

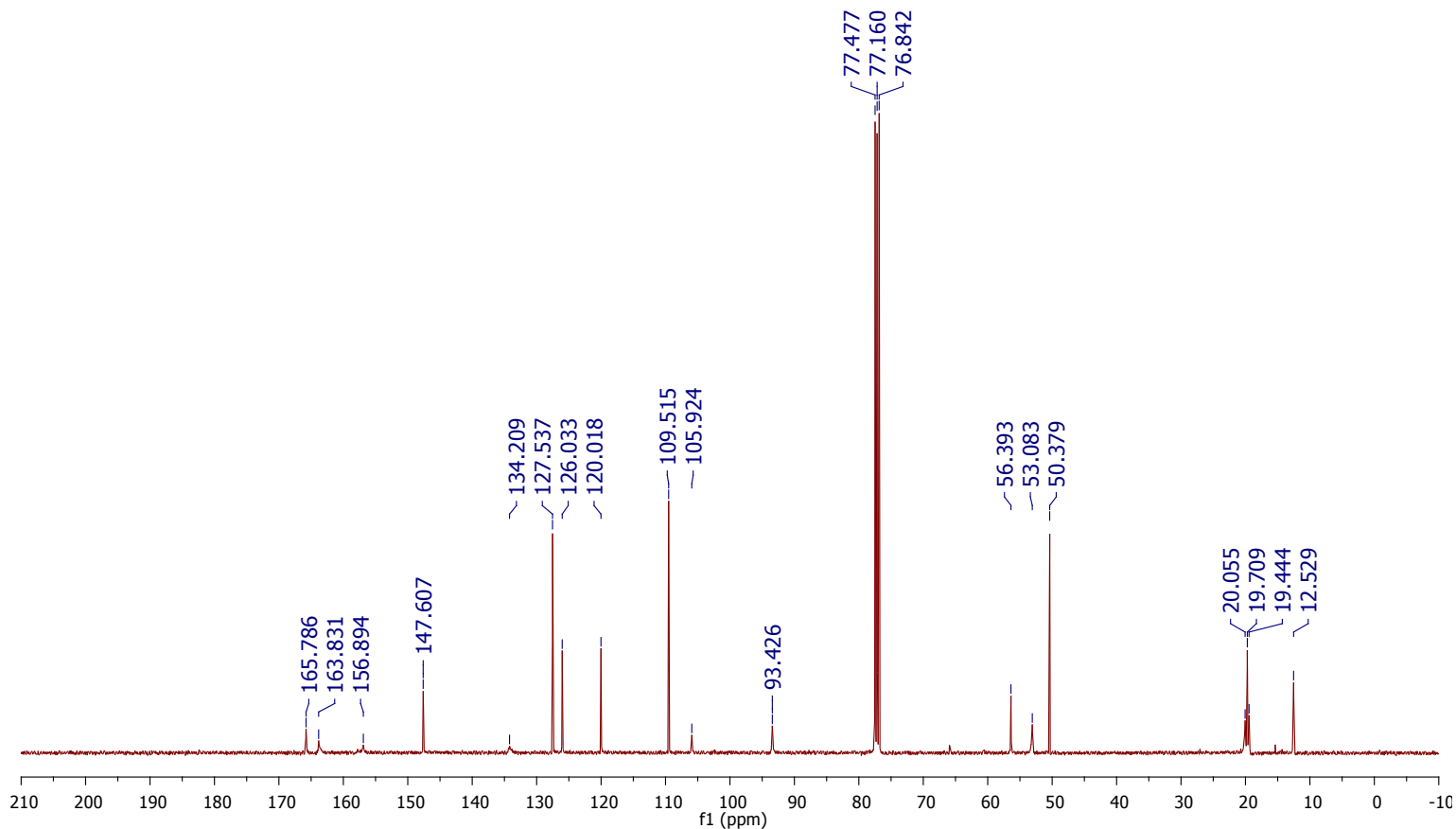


${ }^{1} \mathrm{H}$ NMR of $5 \mathrm{~h}\left(400 \mathrm{MHz}, \mathrm{CDCl}_{3}\right)$

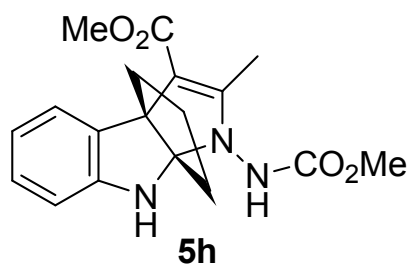

$5 \mathrm{~h}$

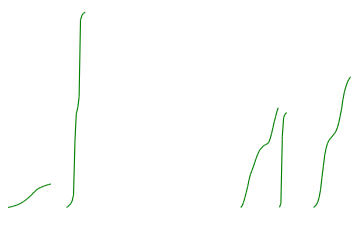

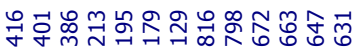

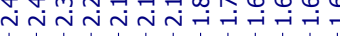
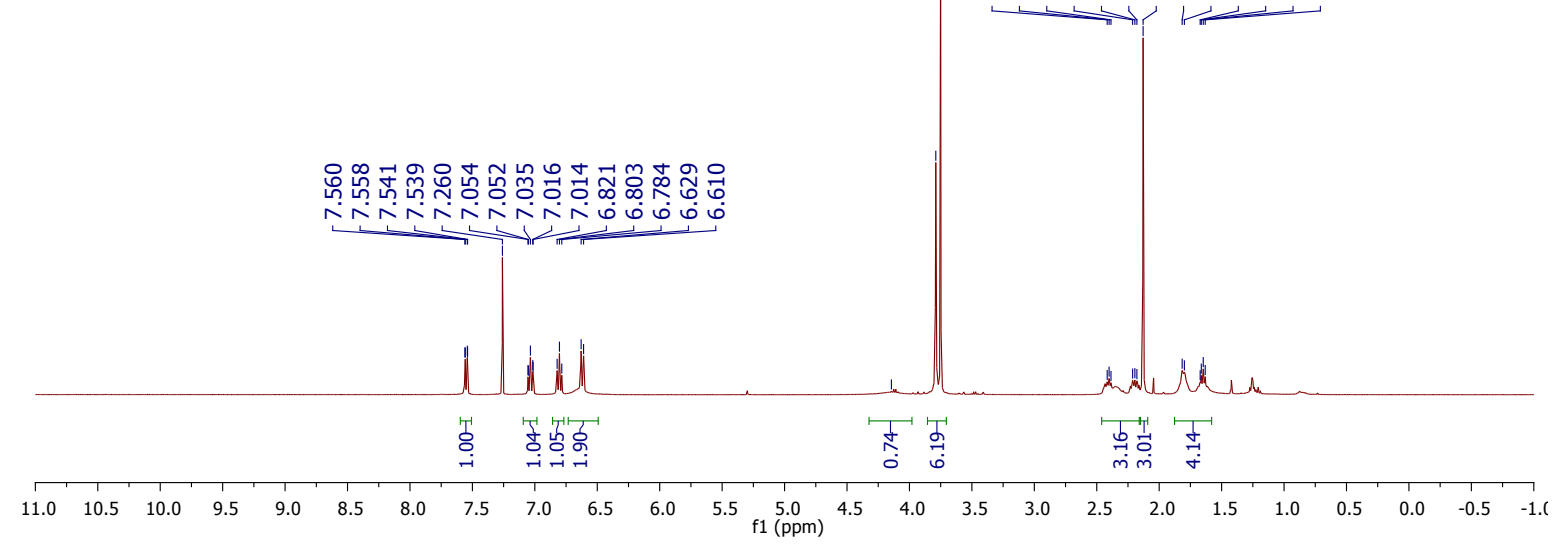

${ }^{13} \mathrm{C}$ NMR of $5 \mathrm{~h}\left(100 \mathrm{MHz}, \mathrm{CDCl}_{3}\right)$

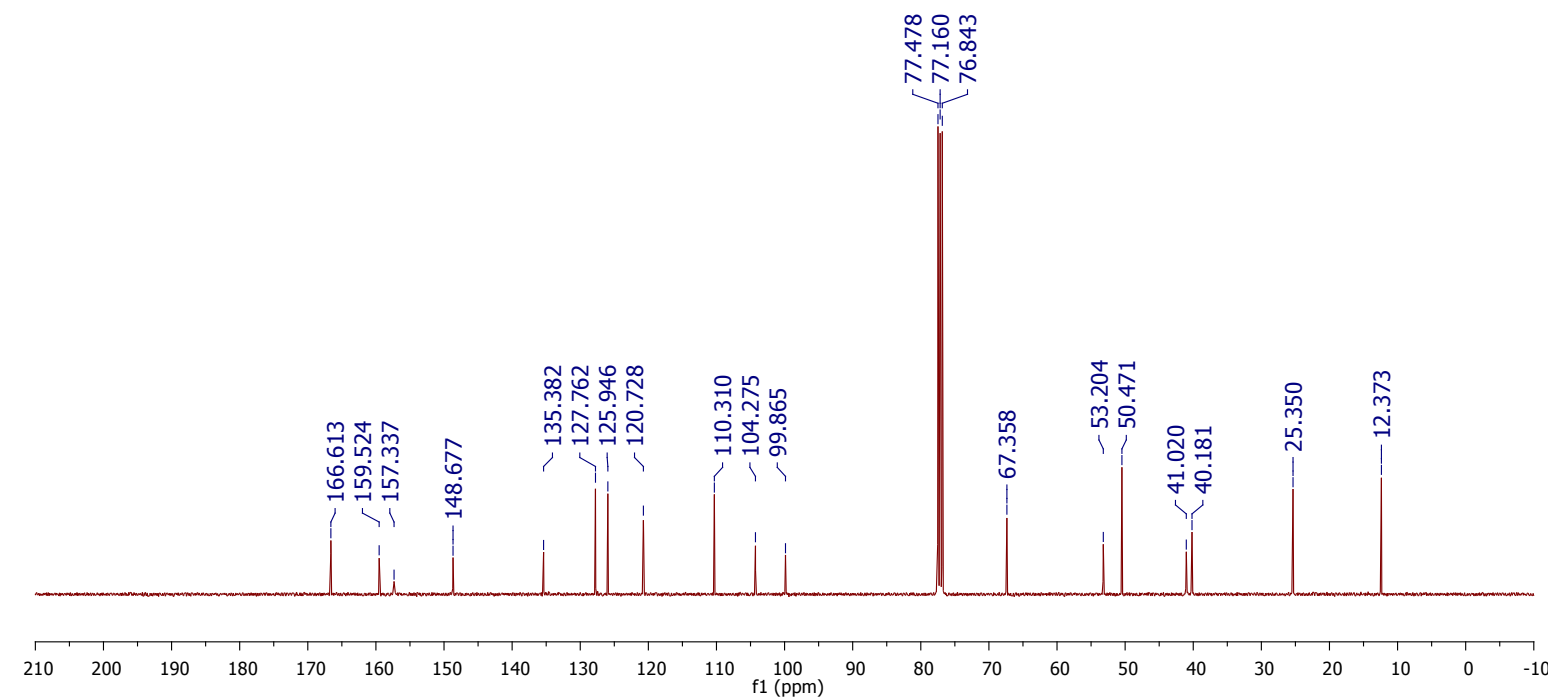


${ }^{1} \mathrm{H}$ NMR of $5 \mathrm{i}$ (400 MHz, DMSO- $\left.d_{6}\right)$<smiles>COC(=O)NN1C(C)=C(C)C2(C)CCC1(C)C2(C)OC</smiles>

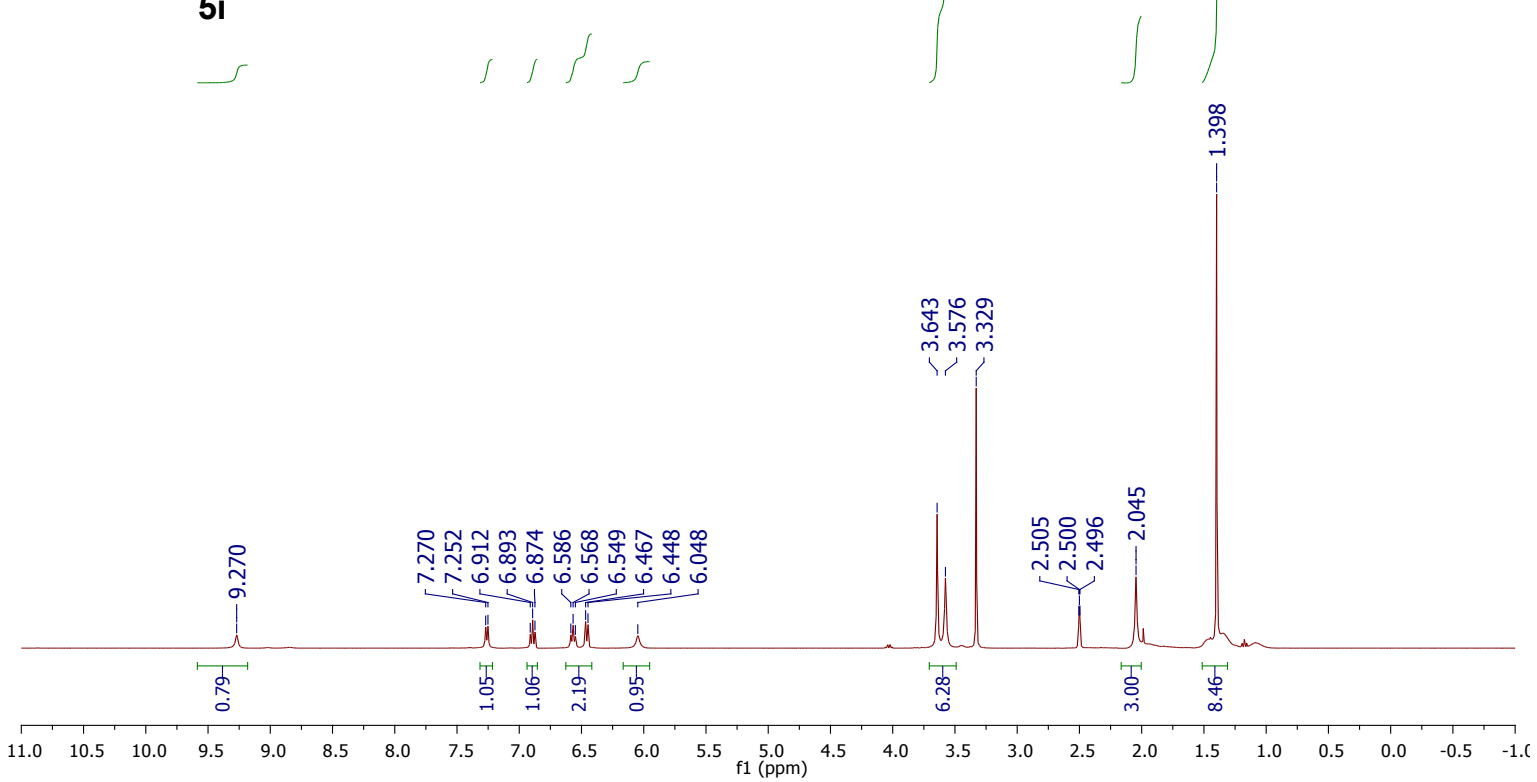

${ }^{13} \mathrm{C}$ NMR of $5 \mathrm{i}\left(100 \mathrm{MHz}, \mathrm{DMSO}-d_{6}\right)$

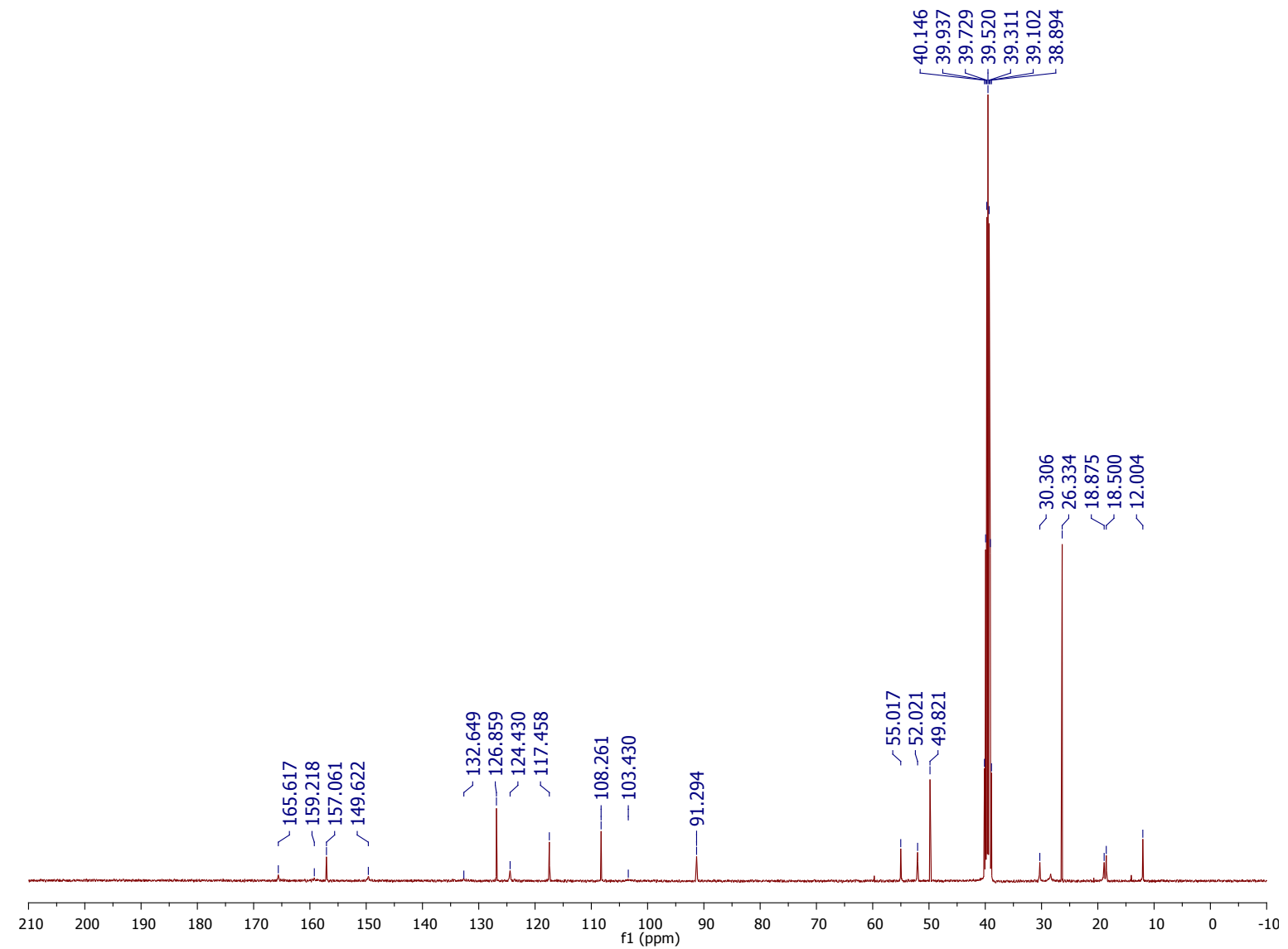


${ }^{1} \mathrm{H}$ NMR of $5 \mathrm{j}$ (400 MHz, DMSO- $\left.d_{6}\right)$<smiles>CCC12C(C(C)=O)=C(C)N[C@]1(C)Nc1ccc(Cl)cc12</smiles>

$5 j$
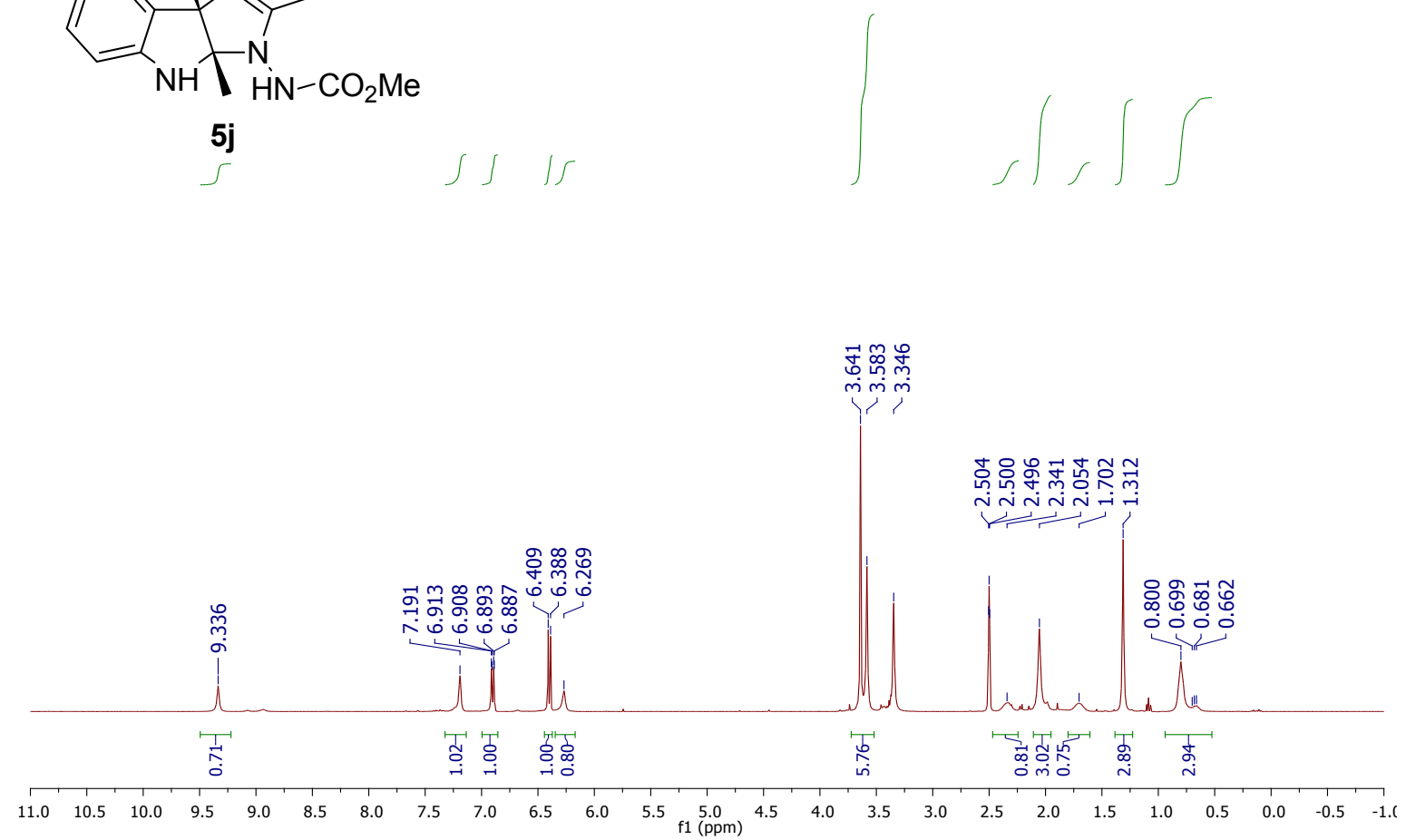

${ }^{13} \mathrm{C}$ NMR of $5 \mathrm{j}$ (100 MHz, DMSO- $\left.d_{6}\right)$

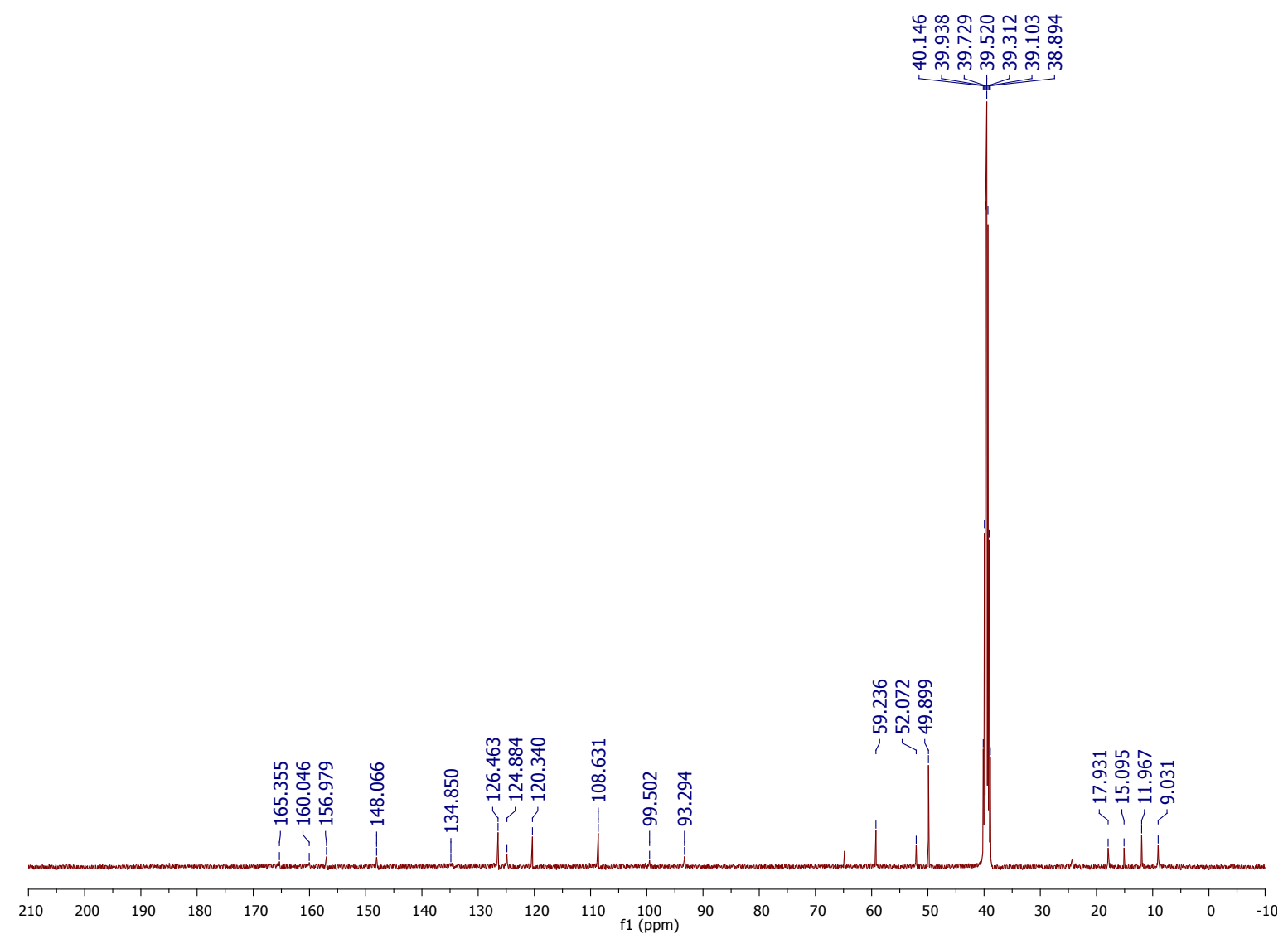


${ }^{1} \mathrm{H}$ NMR of 5k (400 MHz, DMSO- $\left.d_{6}\right)$<smiles>CCC12c3cc(OC)ccc3NC1(C)[C@]1(CC)C(C(C)=O)=C(C)N(NC(C)=O)C21C</smiles>
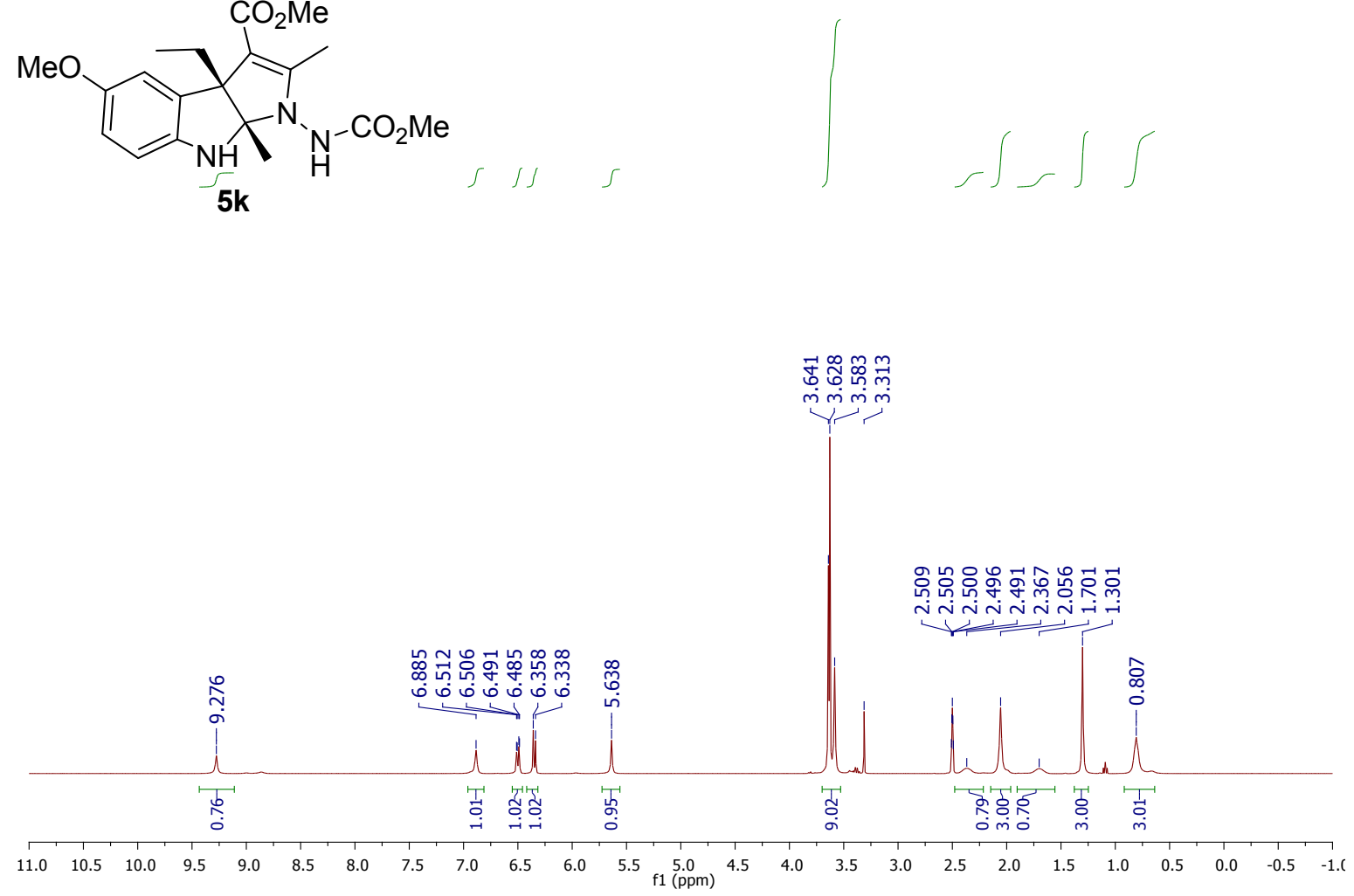

${ }^{13} \mathrm{C}$ NMR of 5k (100 MHz, DMSO- $\left.d_{6}\right)$

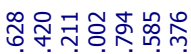

웅ㅎํ

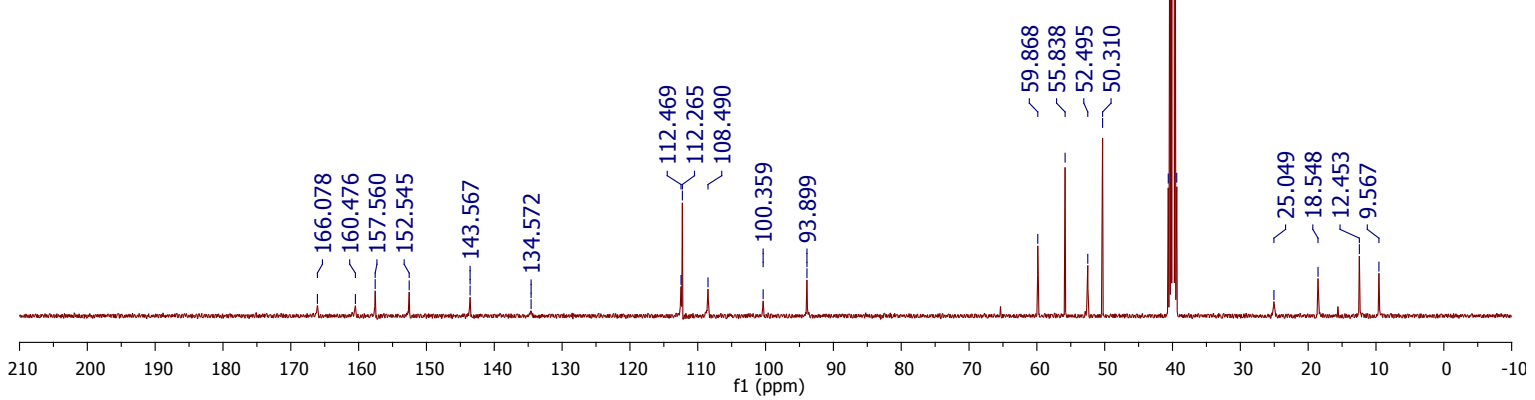


${ }^{1} \mathrm{H}$ NMR of 51 (400 MHz, $\left.\mathrm{CDCl}_{3}\right)$

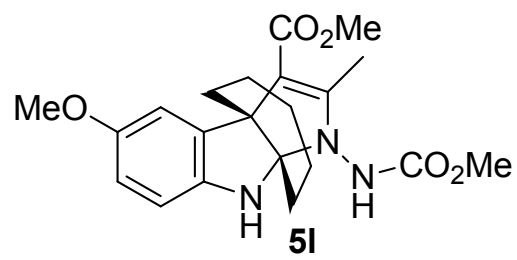

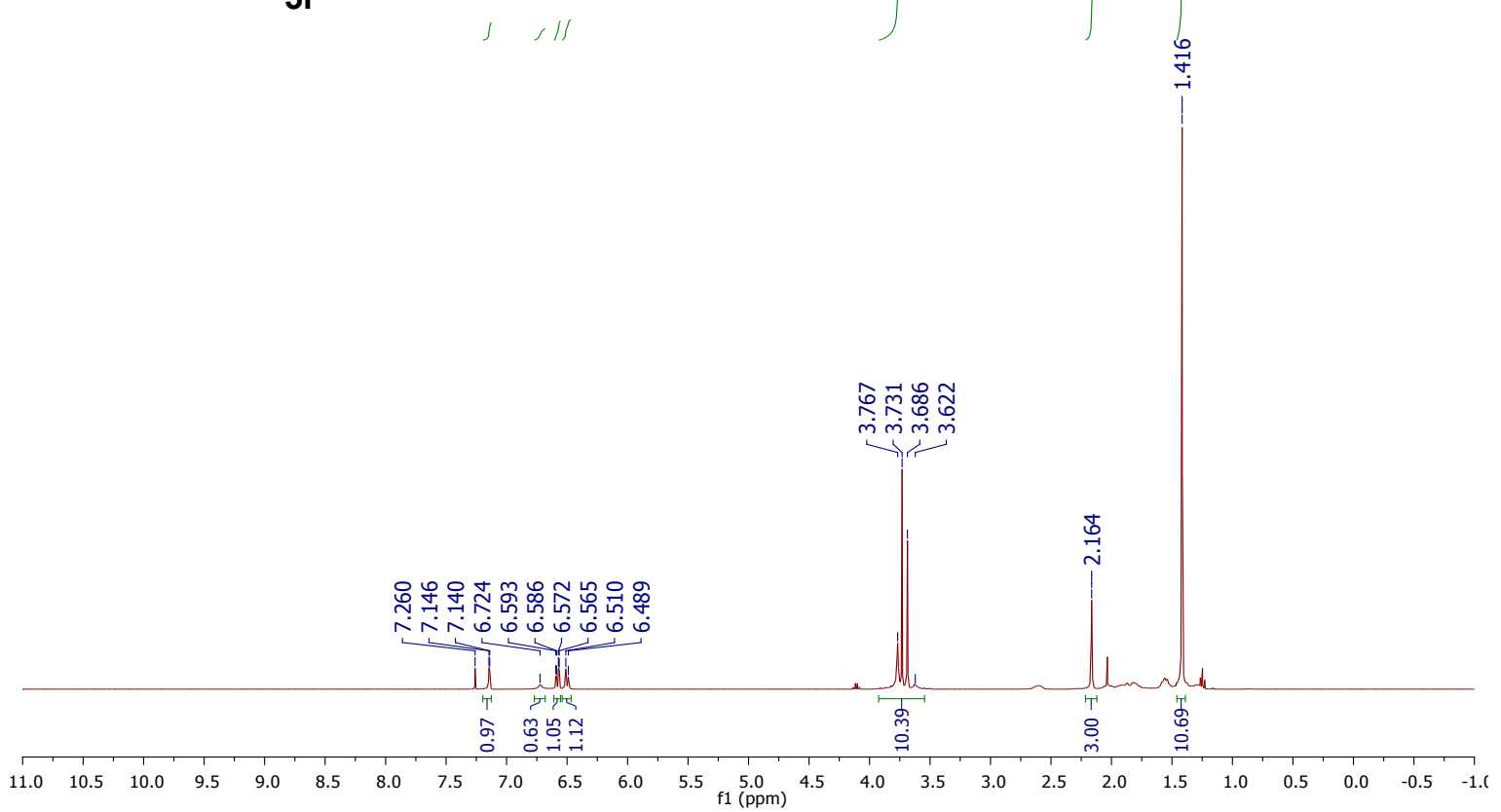

${ }^{13} \mathrm{C}$ NMR of $5 \mathrm{l}\left(100 \mathrm{MHz}, \mathrm{CDCl}_{3}\right)$

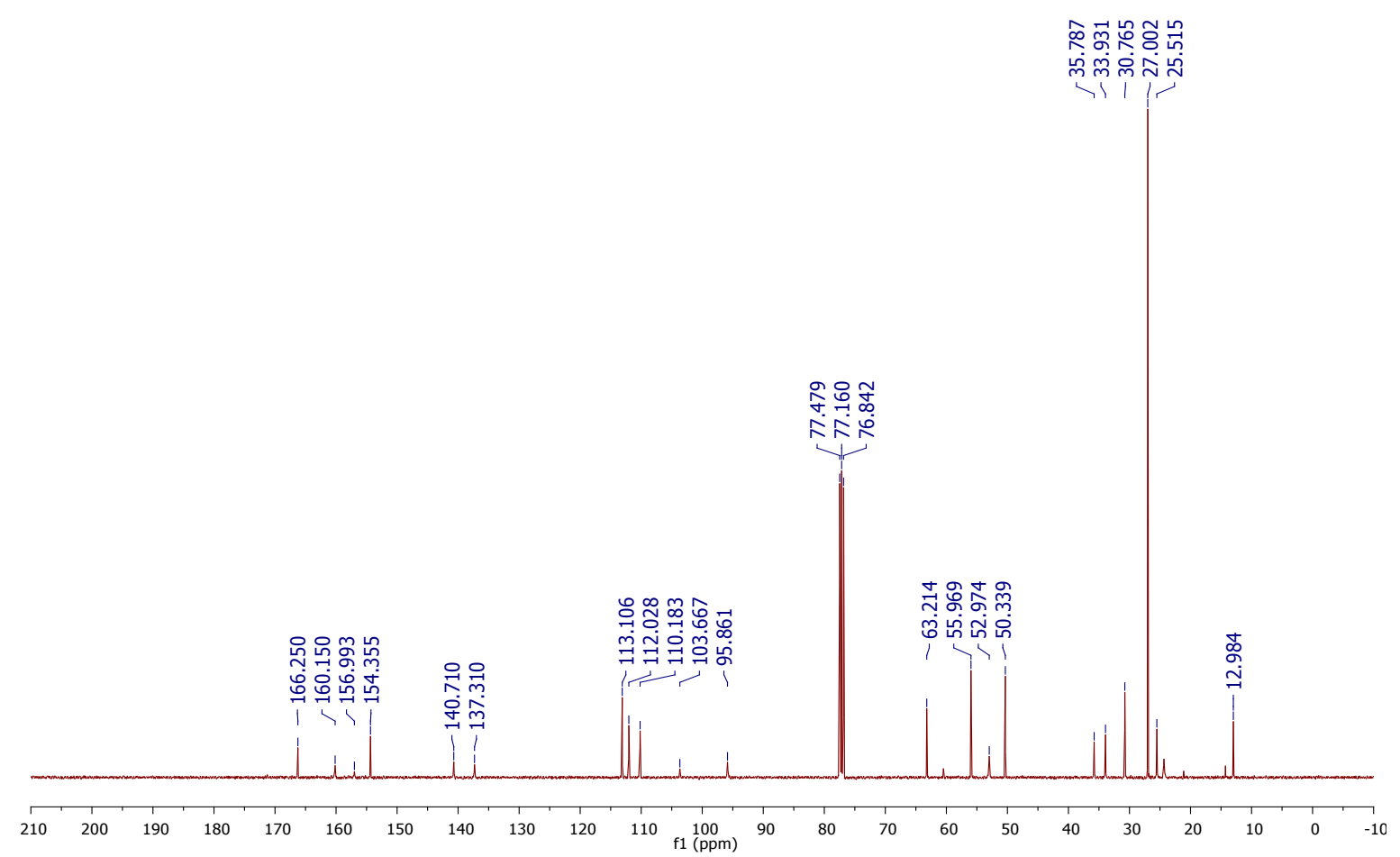


${ }^{1} \mathrm{H}$ NMR of $5 \mathrm{~m}$ (400 MHz, DMSO- $d_{6}$ )<smiles>CCOC(=O)NN1C(C)=C(C(=O)OCC)[C@@]2(C)c3cc(C)ccc3N[C@]12C</smiles>

$5 \mathrm{~m}$

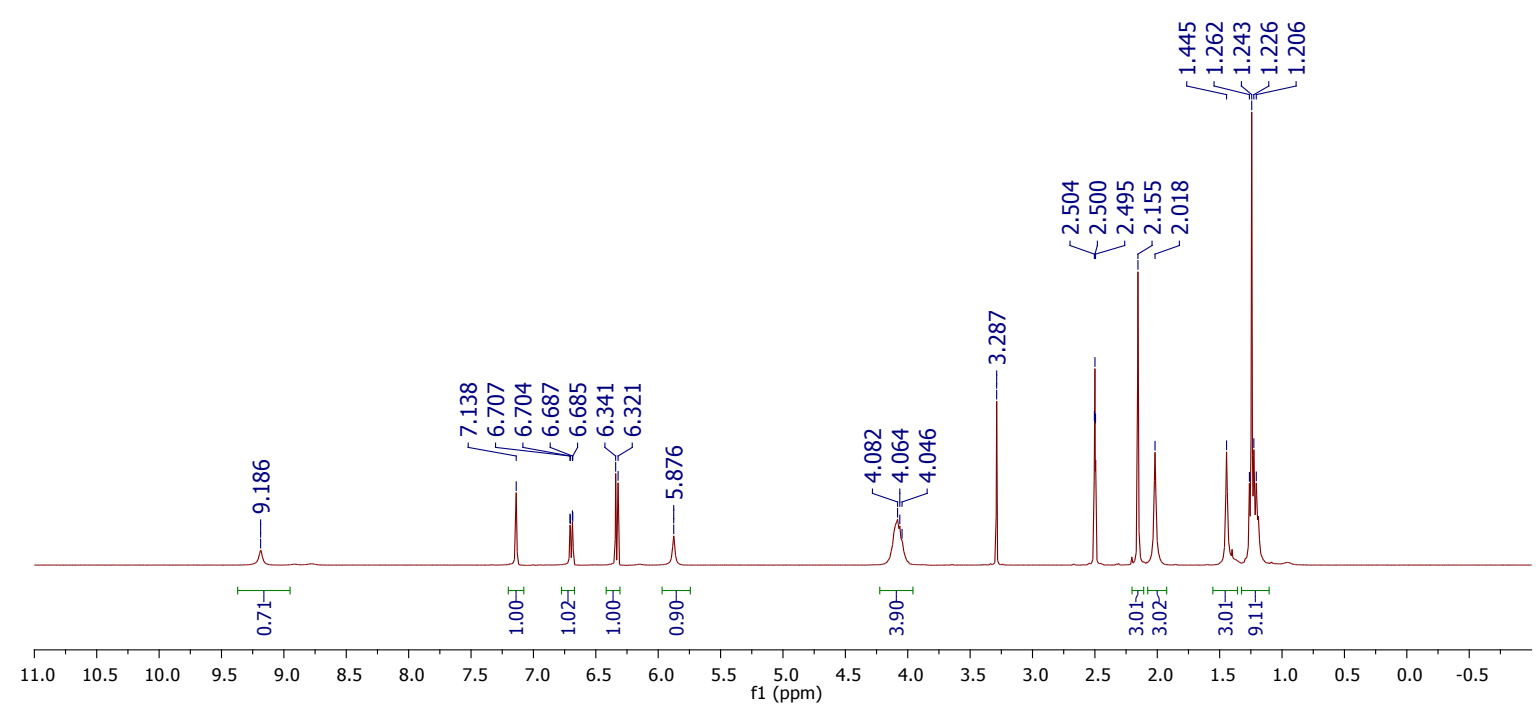

${ }^{13} \mathrm{C}$ NMR of 5m (100 MHz, DMSO- $\left.d_{6}\right)$

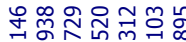 \\ अ}

mim m

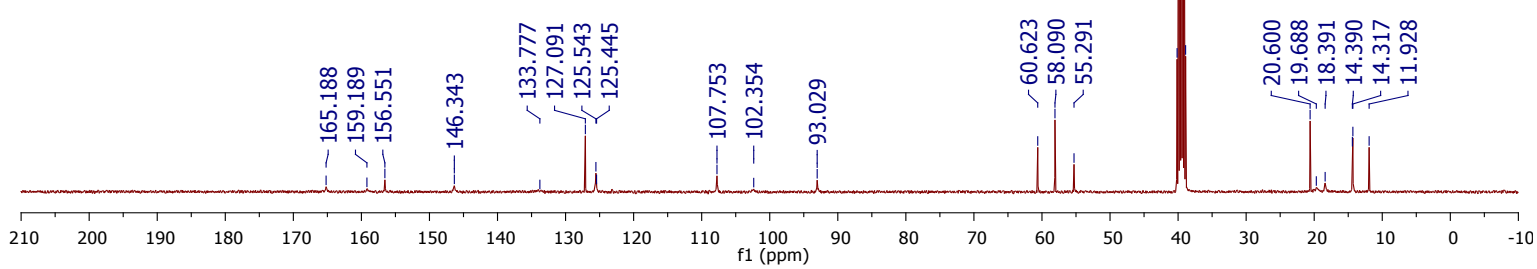


${ }^{1} \mathrm{H}$ NMR of 5n (400 MHz, DMSO- $\left.d_{6}\right)$

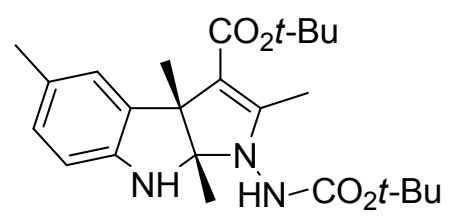

$5 n$

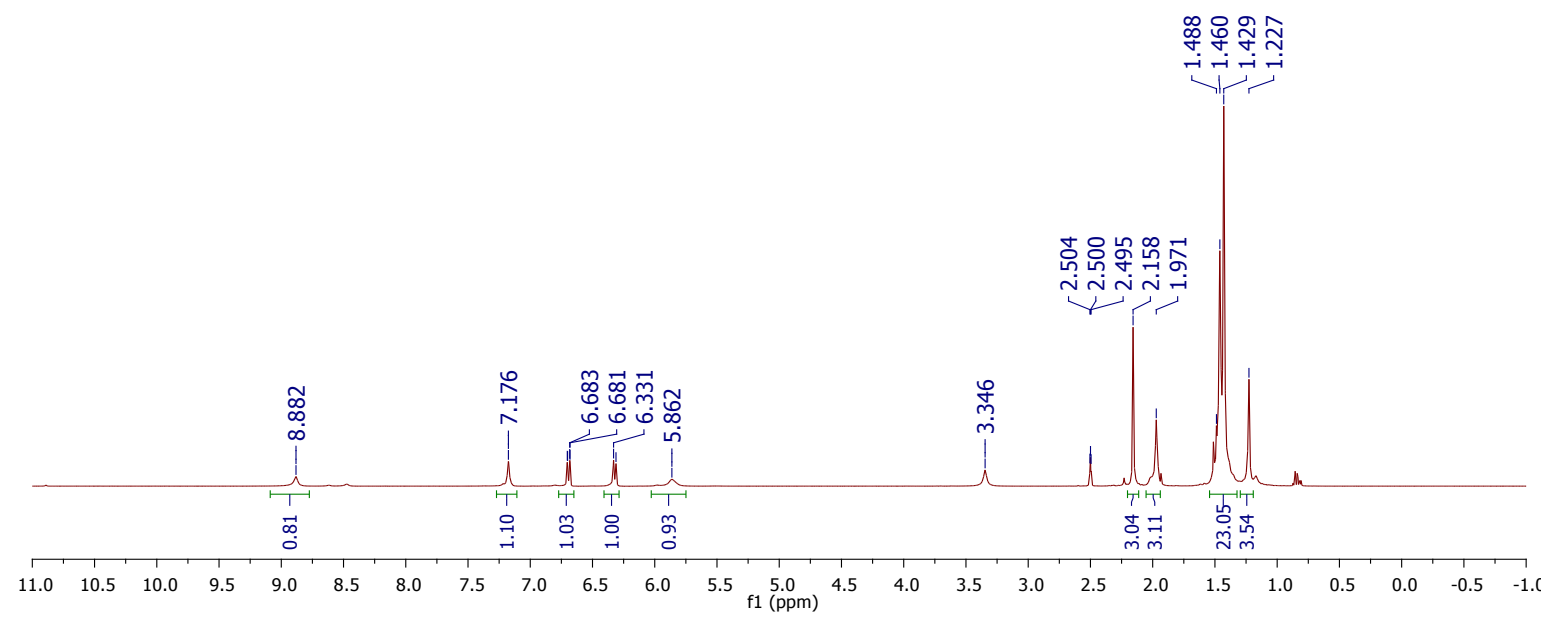

${ }^{13} \mathrm{C}$ NMR of $5 \mathrm{n}\left(100 \mathrm{MHz}\right.$, DMSO- $\left.d_{6}\right)$

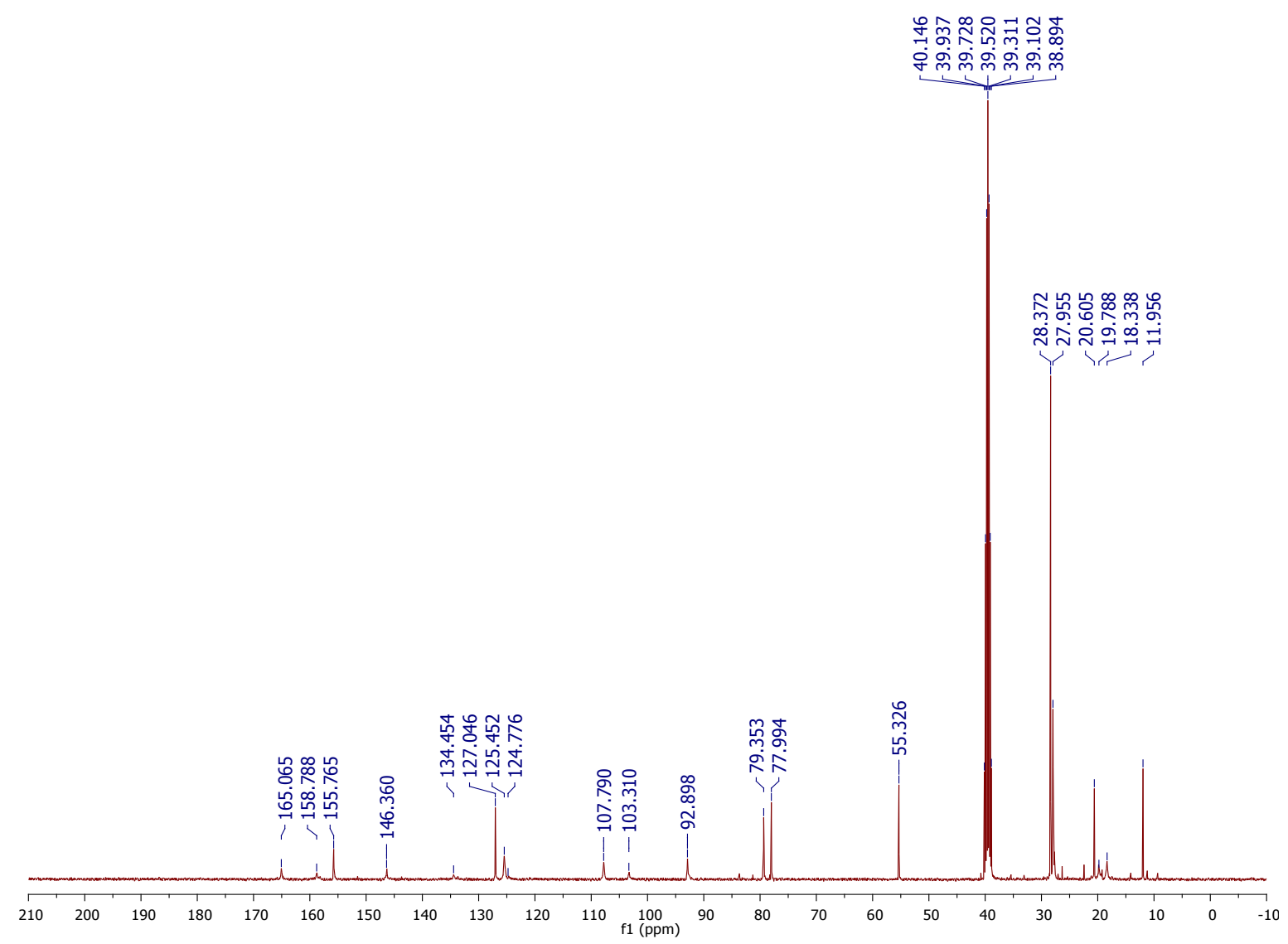


${ }^{1} \mathrm{H}$ NMR of $50\left(400 \mathrm{MHz}, \mathrm{CDCl}_{3}\right)$
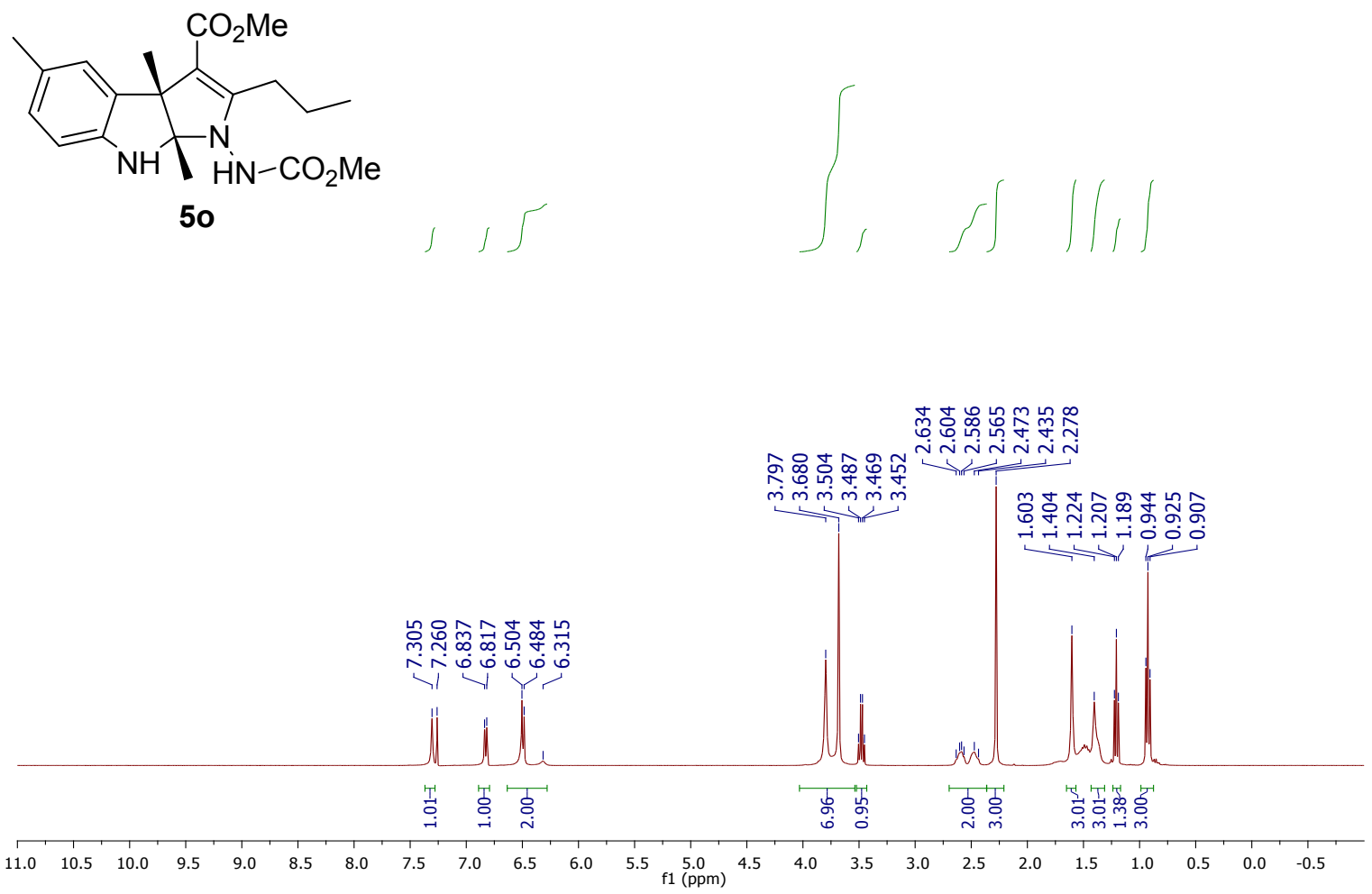

${ }^{13} \mathrm{C}$ NMR of 50 (100 MHz, $\left.\mathrm{CDCl}_{3}\right)$

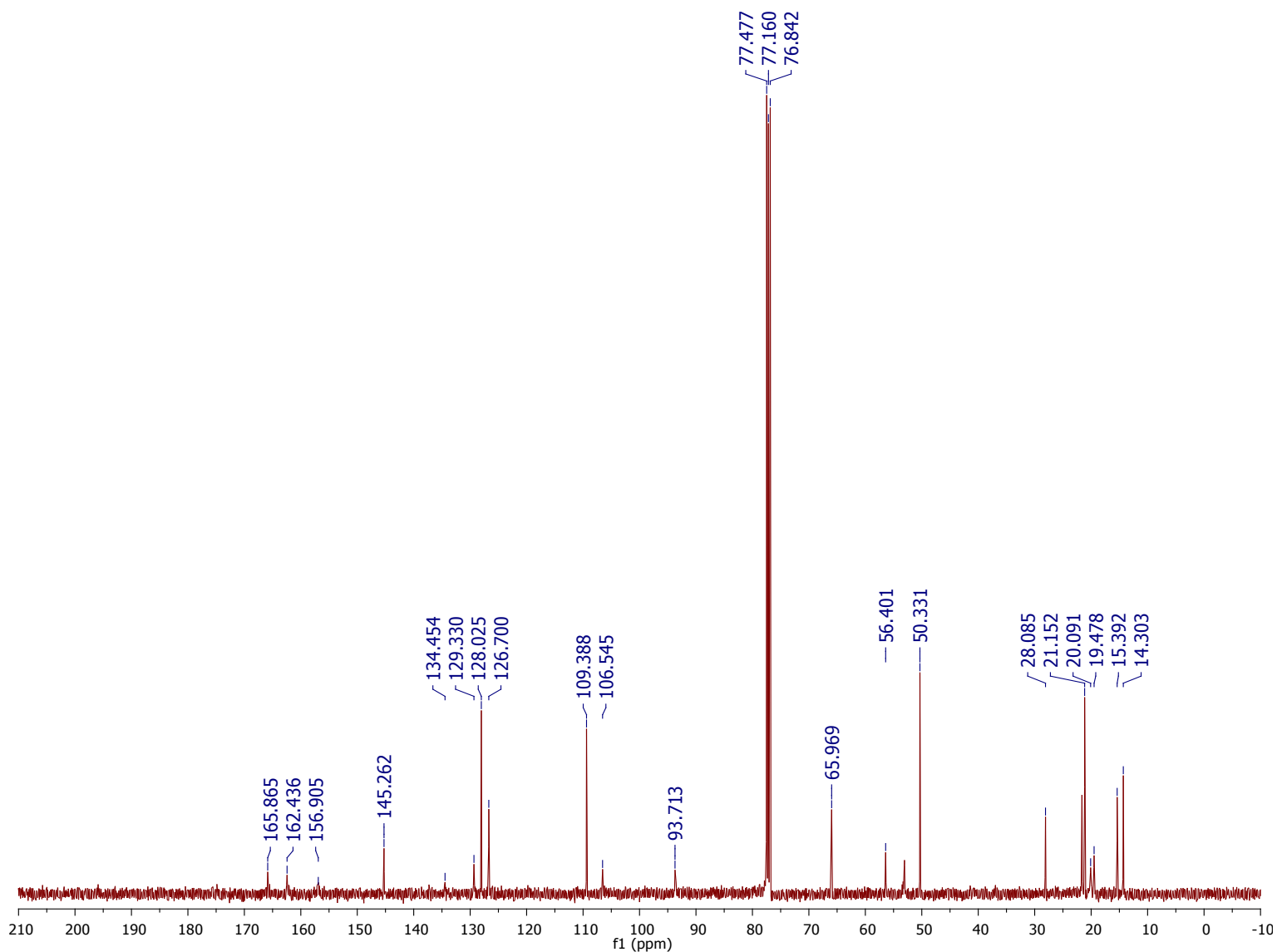


${ }^{1} \mathrm{H}$ NMR of 5p (400 MHz, $\left.\mathrm{CDCl}_{3}\right)$

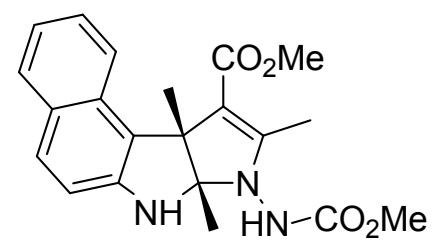

$5 p$
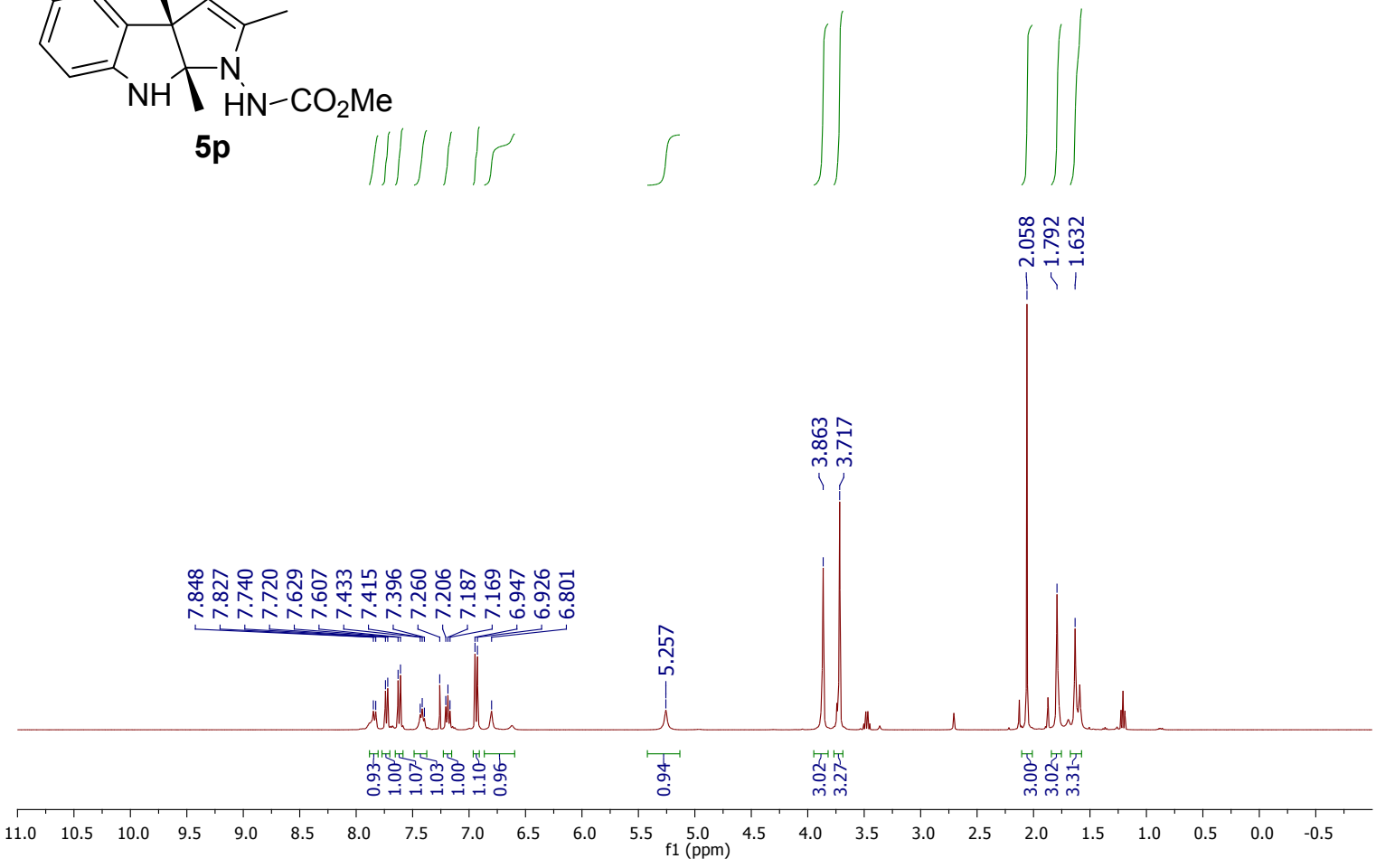

${ }^{13} \mathrm{C}$ NMR of $5 p\left(100 \mathrm{MHz}, \mathrm{CDCl}_{3}\right)$

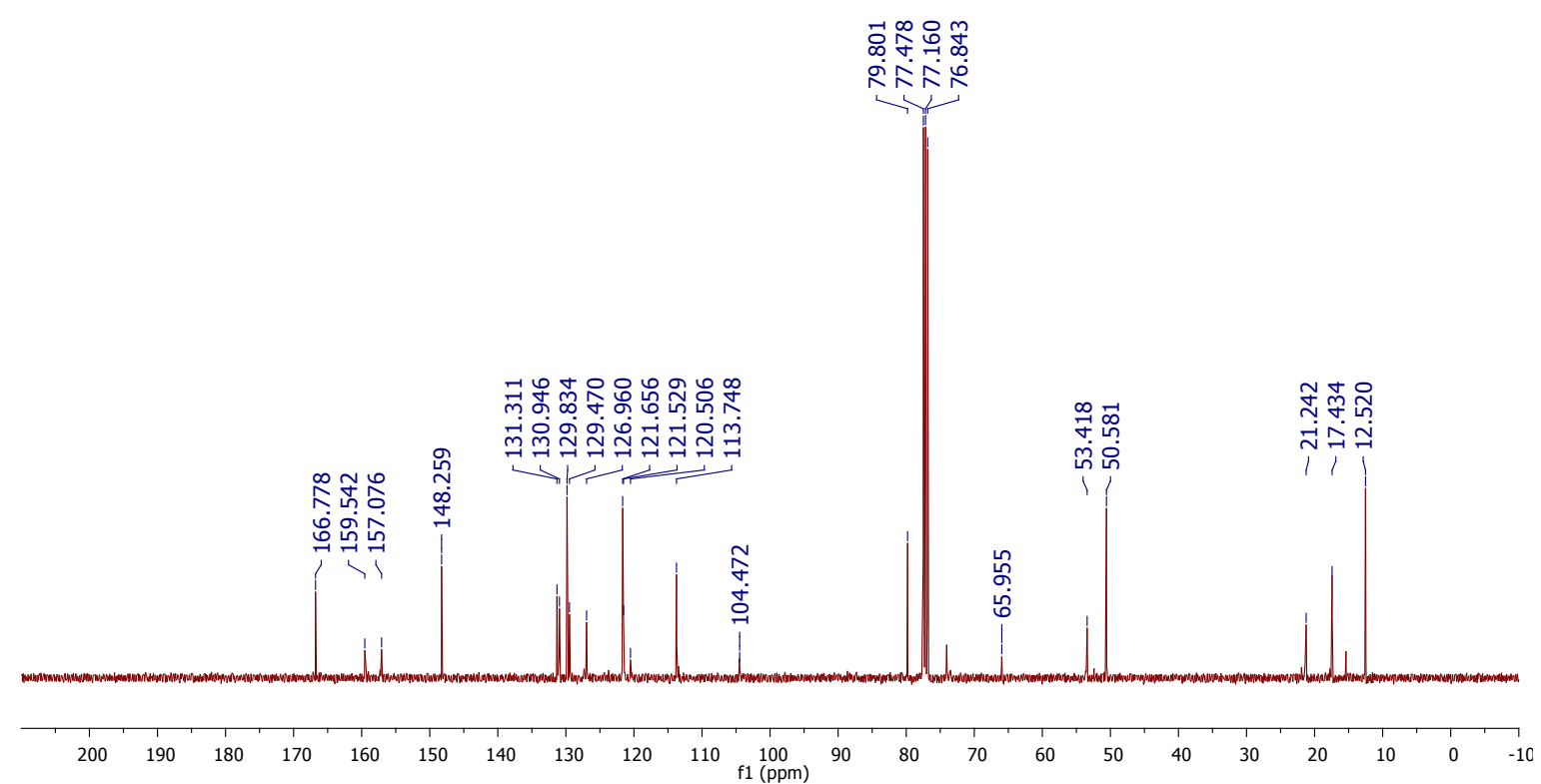


${ }^{1} \mathrm{H}$ NMR of 5q (400 MHz, DMSO-d ${ }_{6}$ )

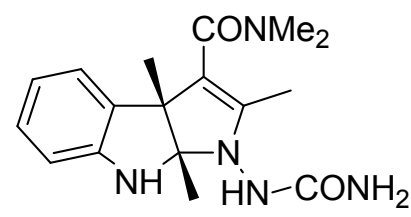

$5 q$

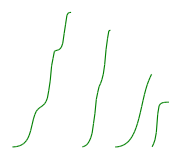

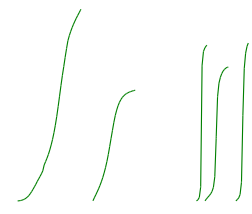

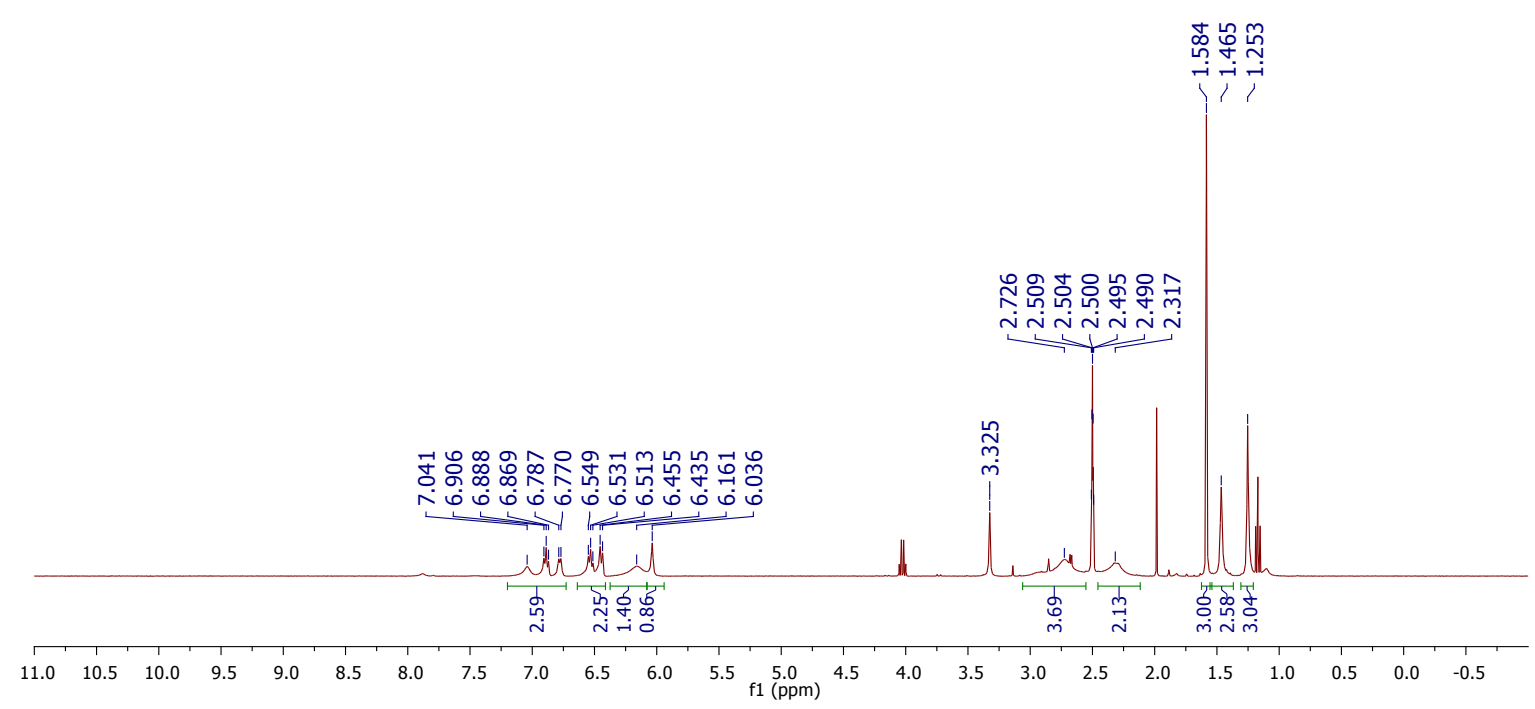

${ }^{13} \mathrm{C}$ NMR of 5q (100 MHz, DMSO- $\left.d_{6}\right)$

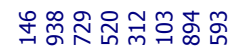

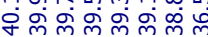

म m m m

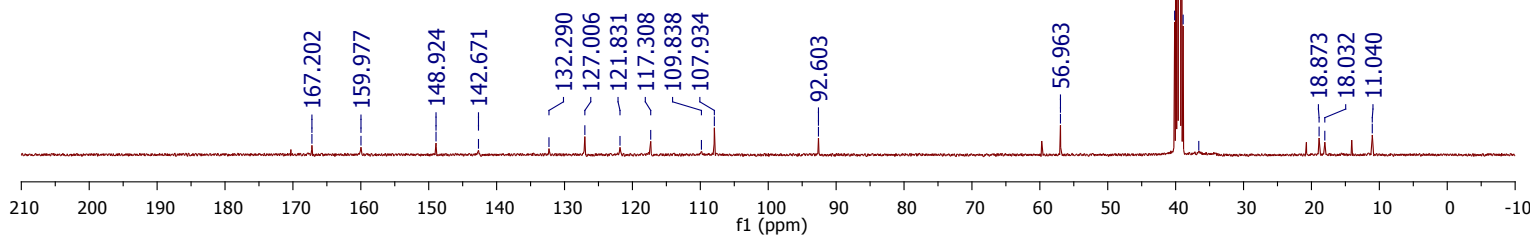


${ }^{1} \mathrm{H}$ NMR of 5 r (400 MHz, DMSO-d $\left.{ }_{6}\right)$

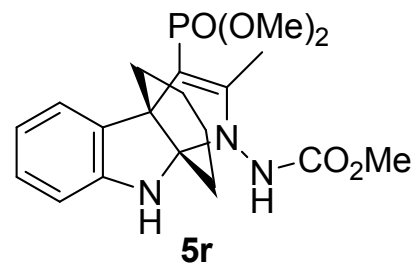
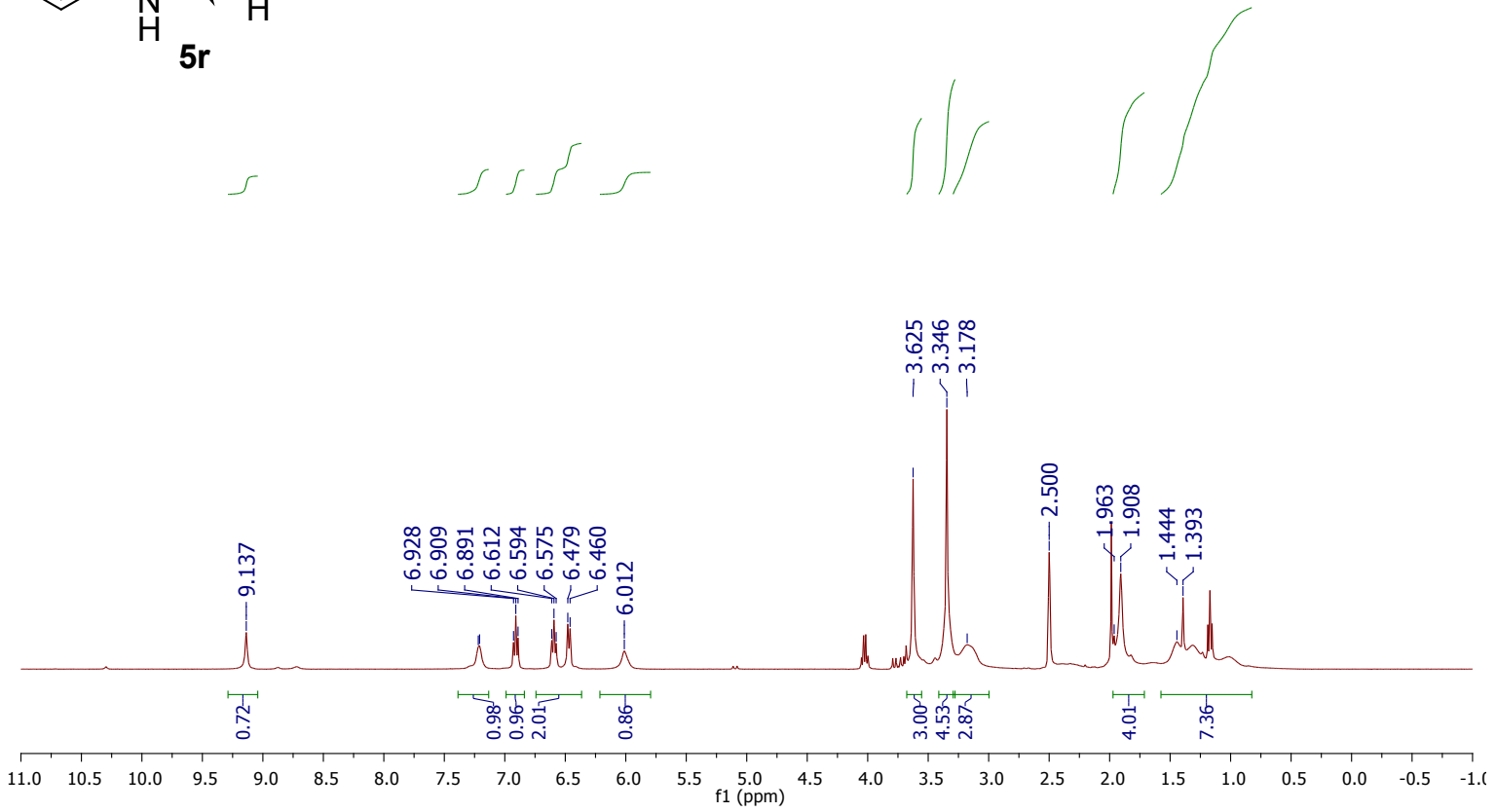

${ }^{13} \mathrm{C}$ NMR of $5 \mathrm{r}$ (100 MHz, DMSO- $\left.d_{6}\right)$

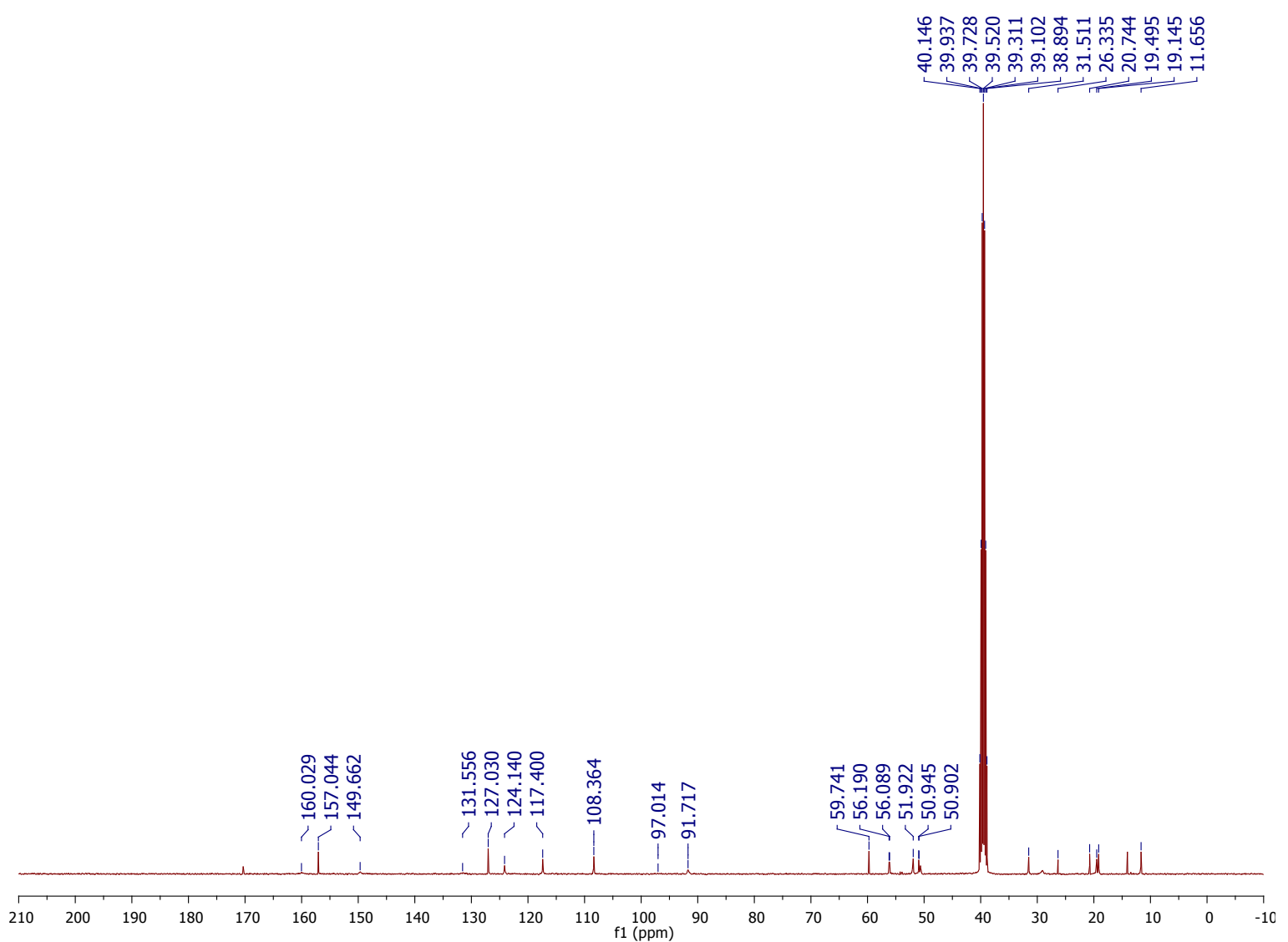


${ }^{1} \mathrm{H}$ NMR of $5 \mathrm{~s}$ (400 MHz, DMSO- $\left.d_{6}\right)$<smiles>CC(=O)NC1=C(C)[C@@]2(C)c3ccccc3N(C)[C@]2(C)N1</smiles>

5s
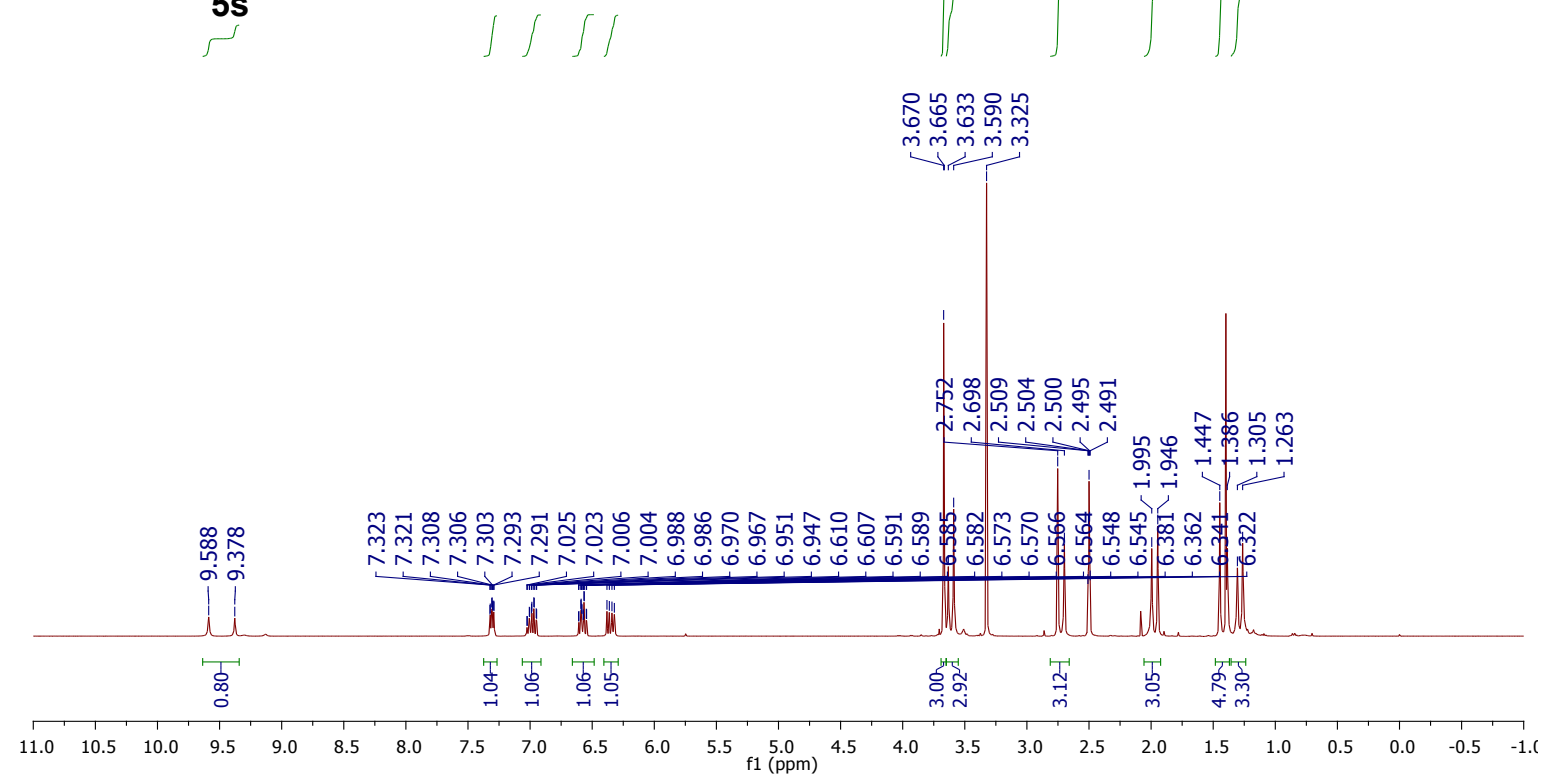

${ }^{13} \mathrm{C}$ NMR of 5s (100 MHz, DMSO- $\left.d_{6}\right)$

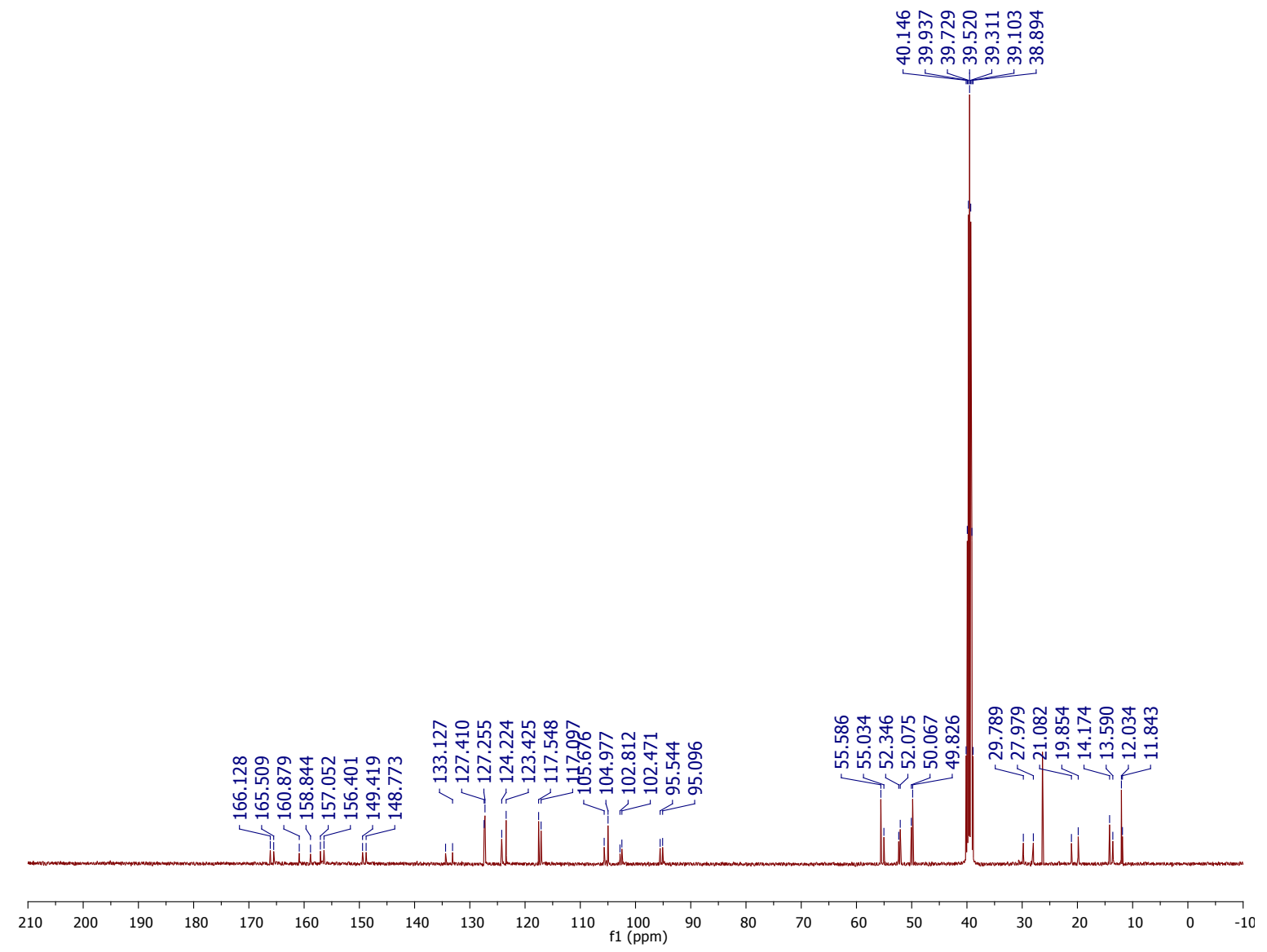


${ }^{1} \mathrm{H}$ NMR of 4a (400 MHz, $\left.\mathrm{CDCl}_{3}\right)$
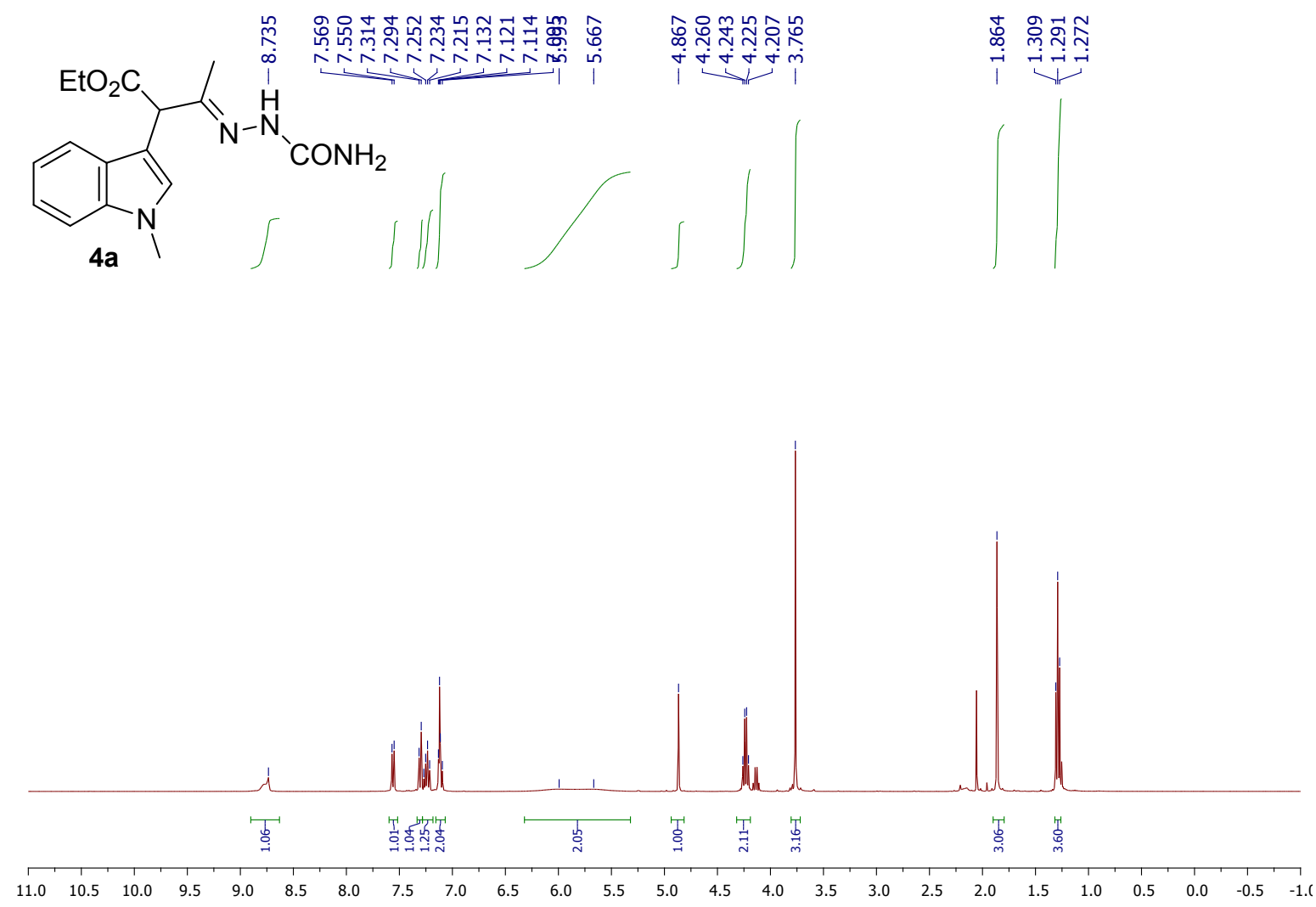

${ }^{13} \mathrm{C}$ NMR of $4 a$ (100 $\left.\mathrm{MHz}, \mathrm{CDCl}_{3}\right)$

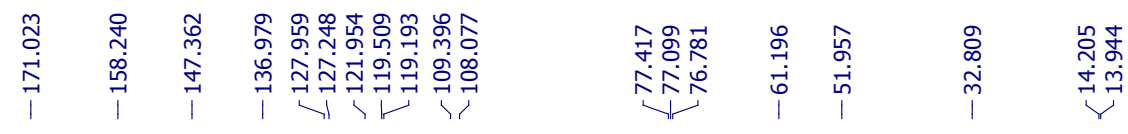

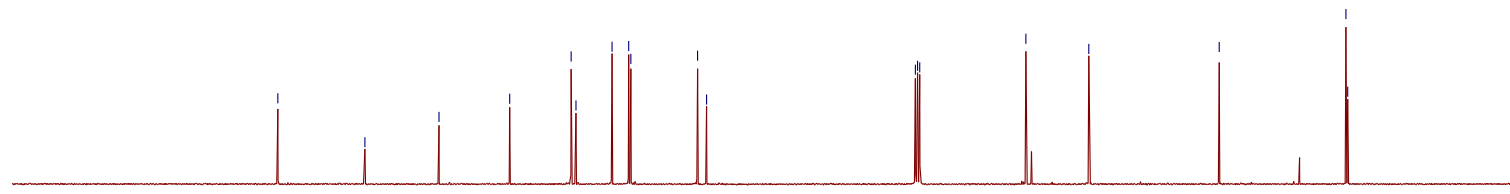

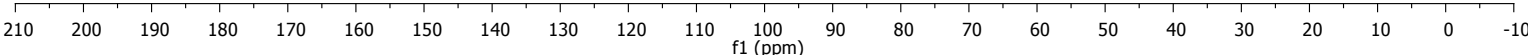


${ }^{1} \mathrm{H}$ NMR of $4 \mathrm{~b}$ (400 MHz, DMSO- $d_{6}$ )

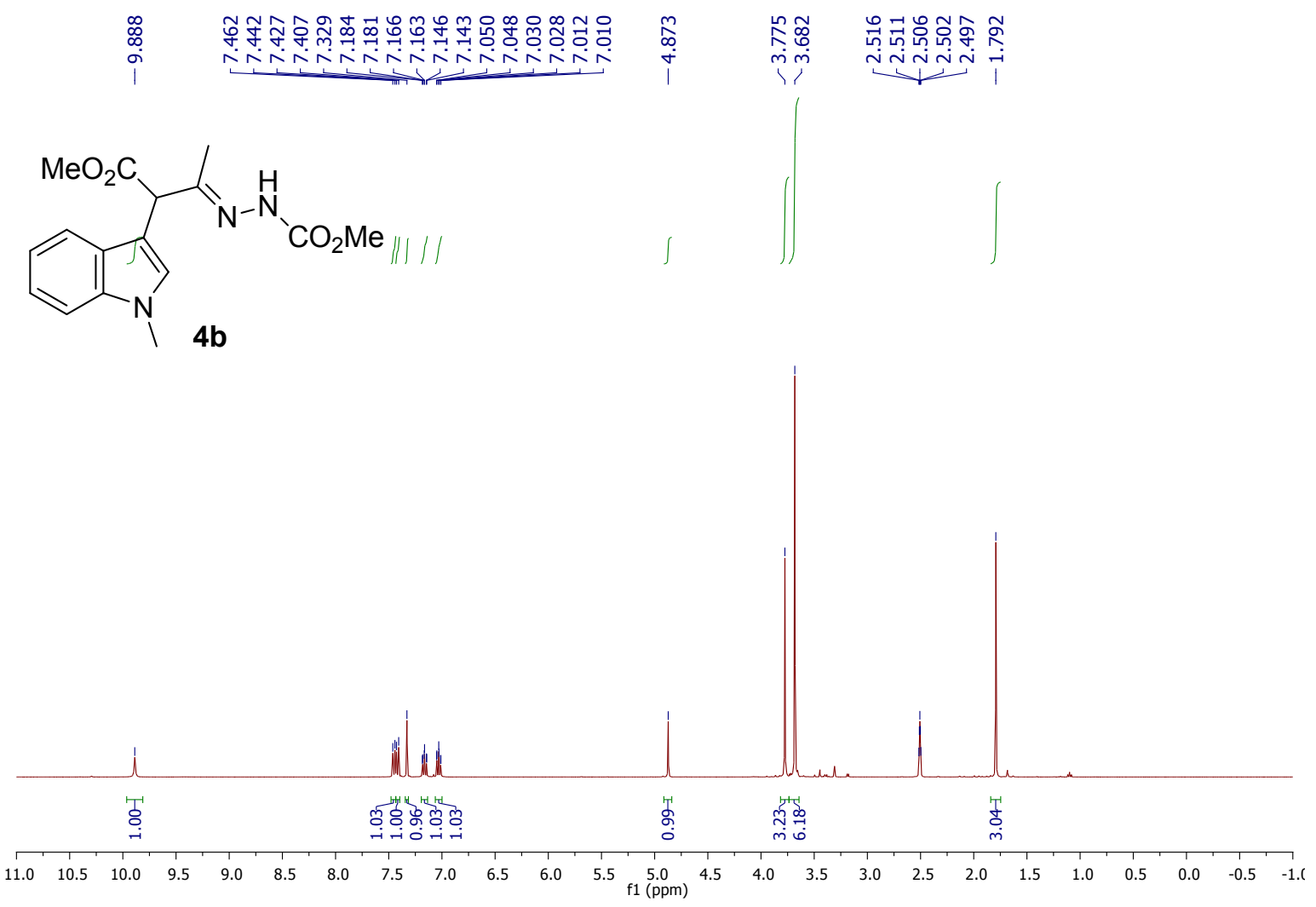

${ }^{13} \mathrm{C}$ NMR of $4 \mathrm{~b}$ (100 MHz, DMSO- $\left.d_{6}\right)$

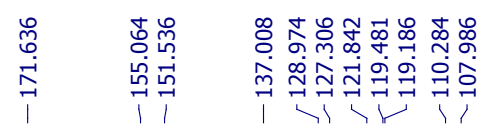

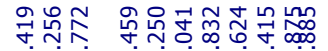

认่

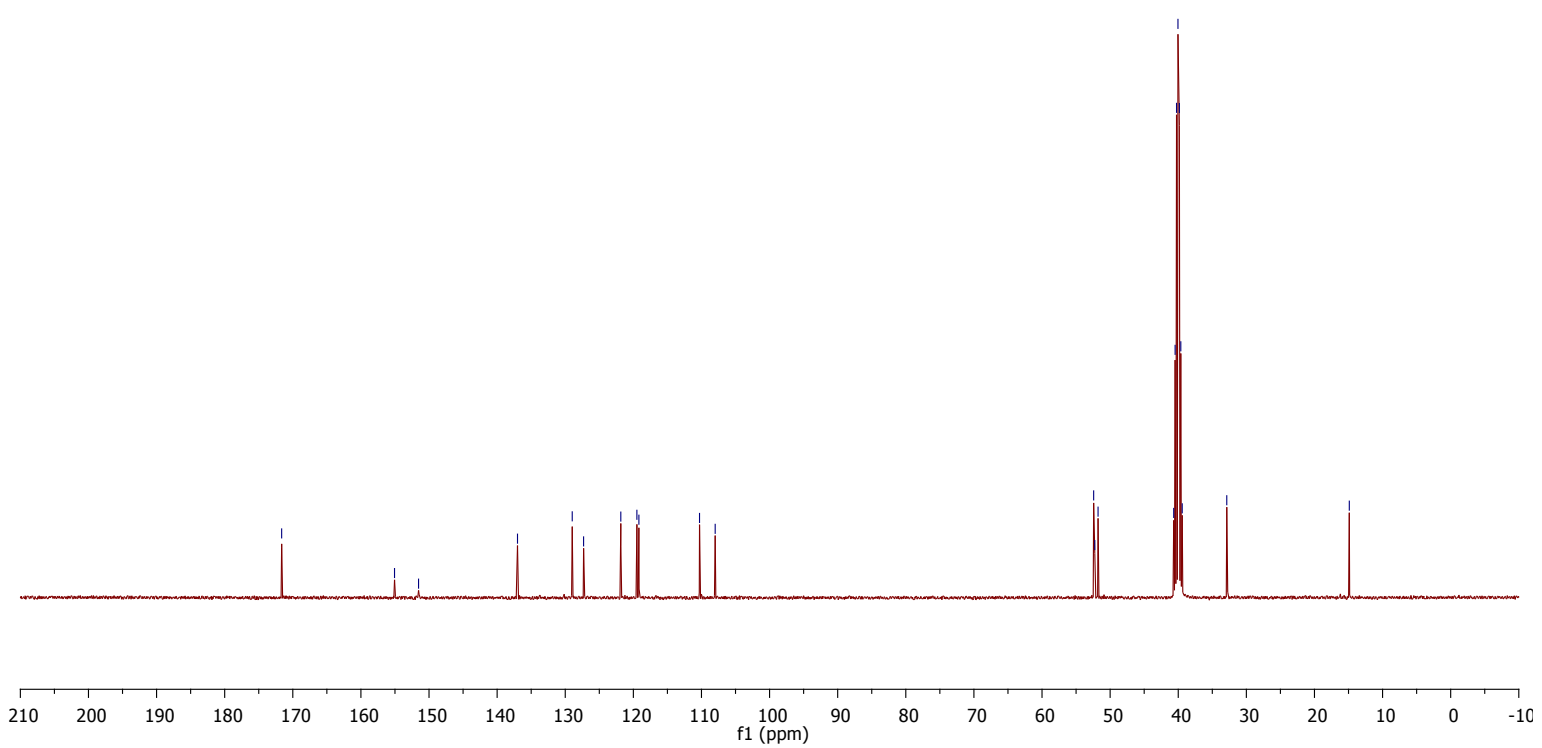


${ }^{1} \mathrm{H}$ NMR of $4 \mathrm{c}\left(400 \mathrm{MHz}\right.$, DMSO- $\left.d_{6}\right)$
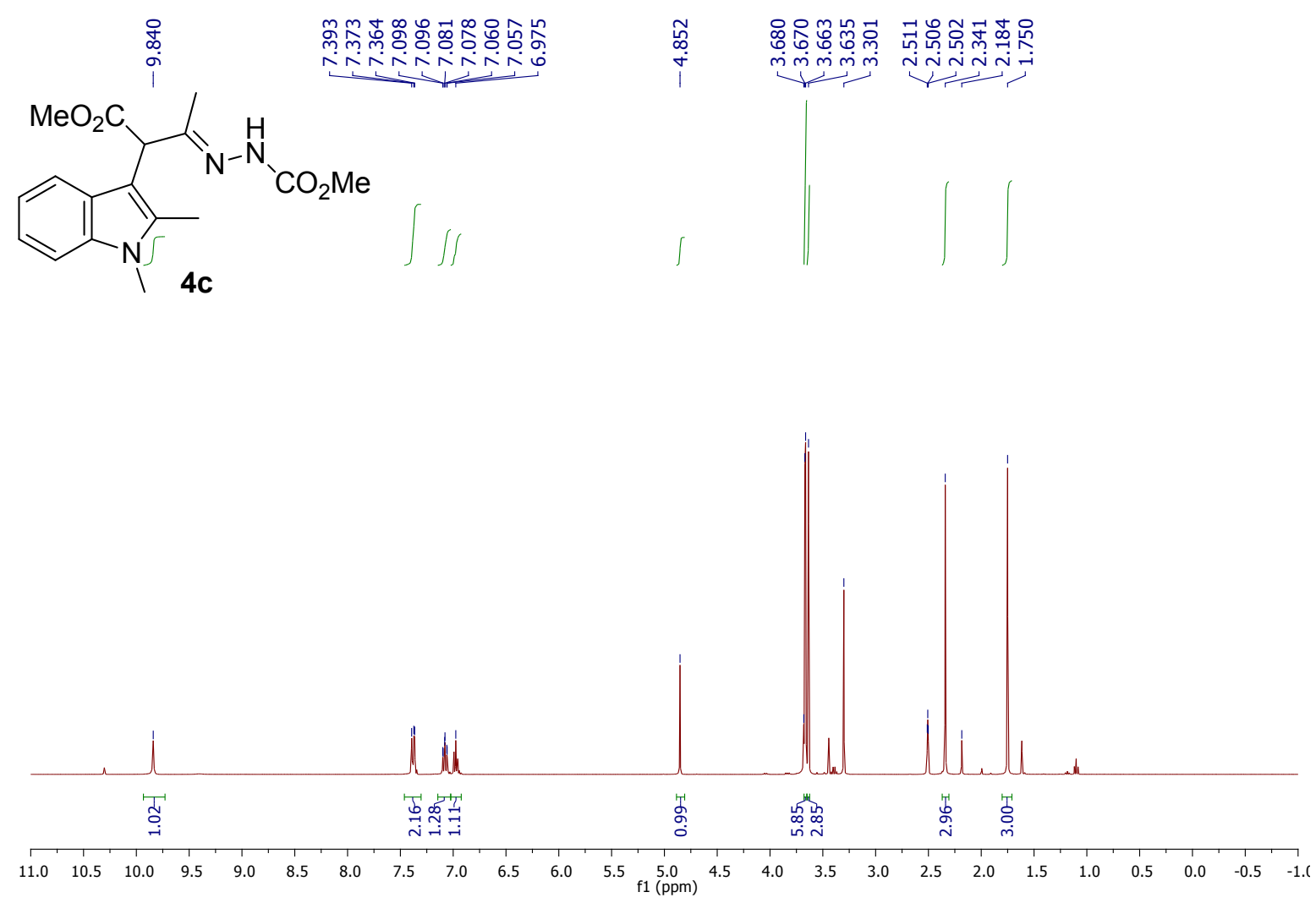

${ }^{13} \mathrm{C}$ NMR of $4 \mathrm{c}\left(100 \mathrm{MHz}, \mathrm{DMSO}-d_{6}\right)$

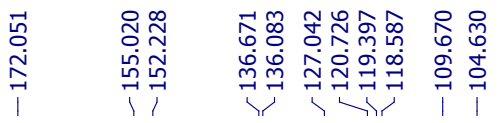

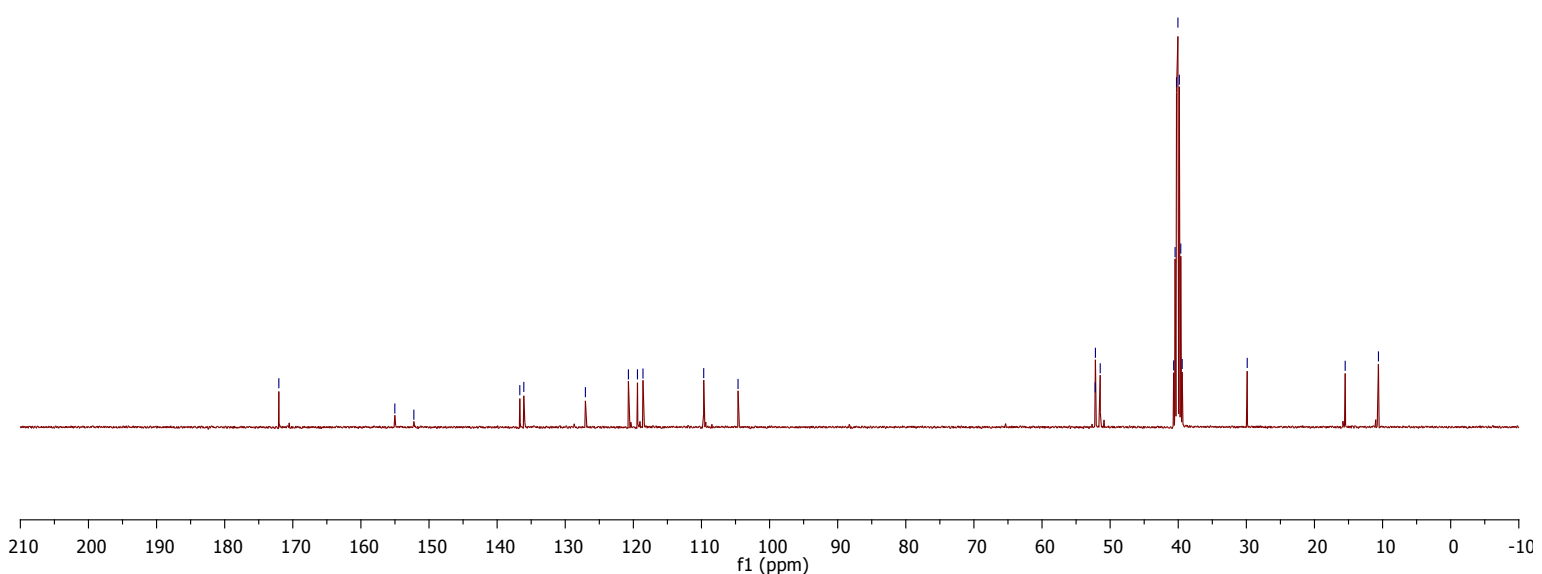


${ }^{1} \mathrm{H}$ NMR of 4d (400 MHz, DMSO-d $\left.)_{6}\right)$<smiles>CCOc1cccc2c(C)n(C)c(C)c12</smiles>

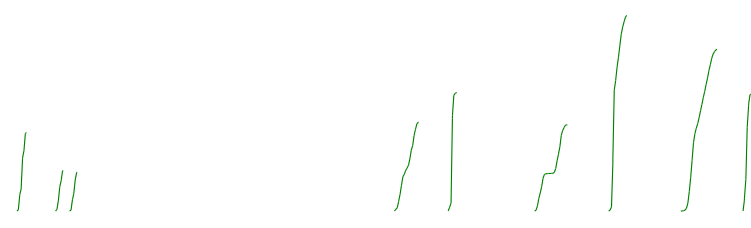

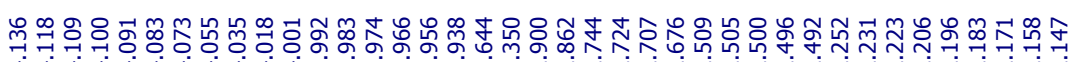

4d

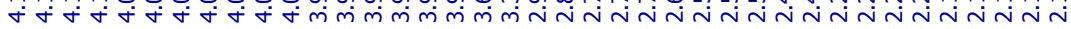

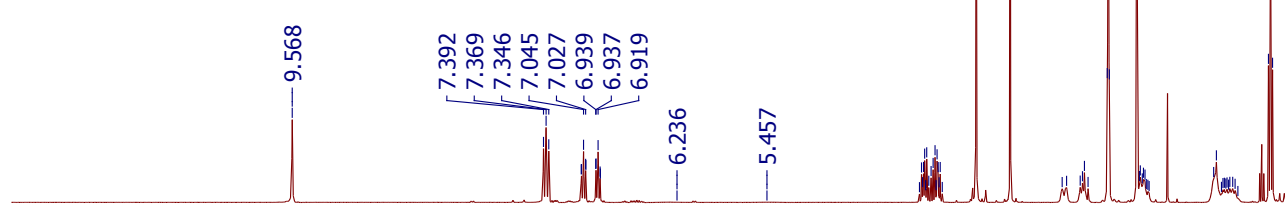

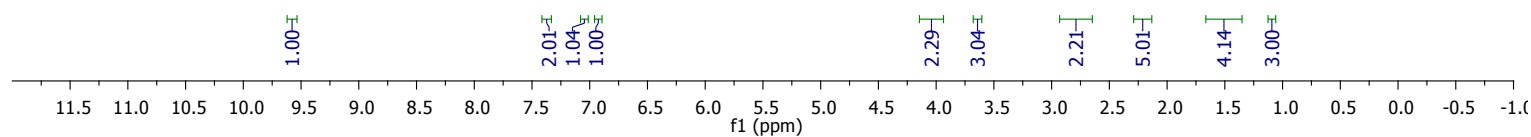

${ }^{13}$ C NMR of 4d (100 MHz, DMSO- $\left.d_{6}\right)$

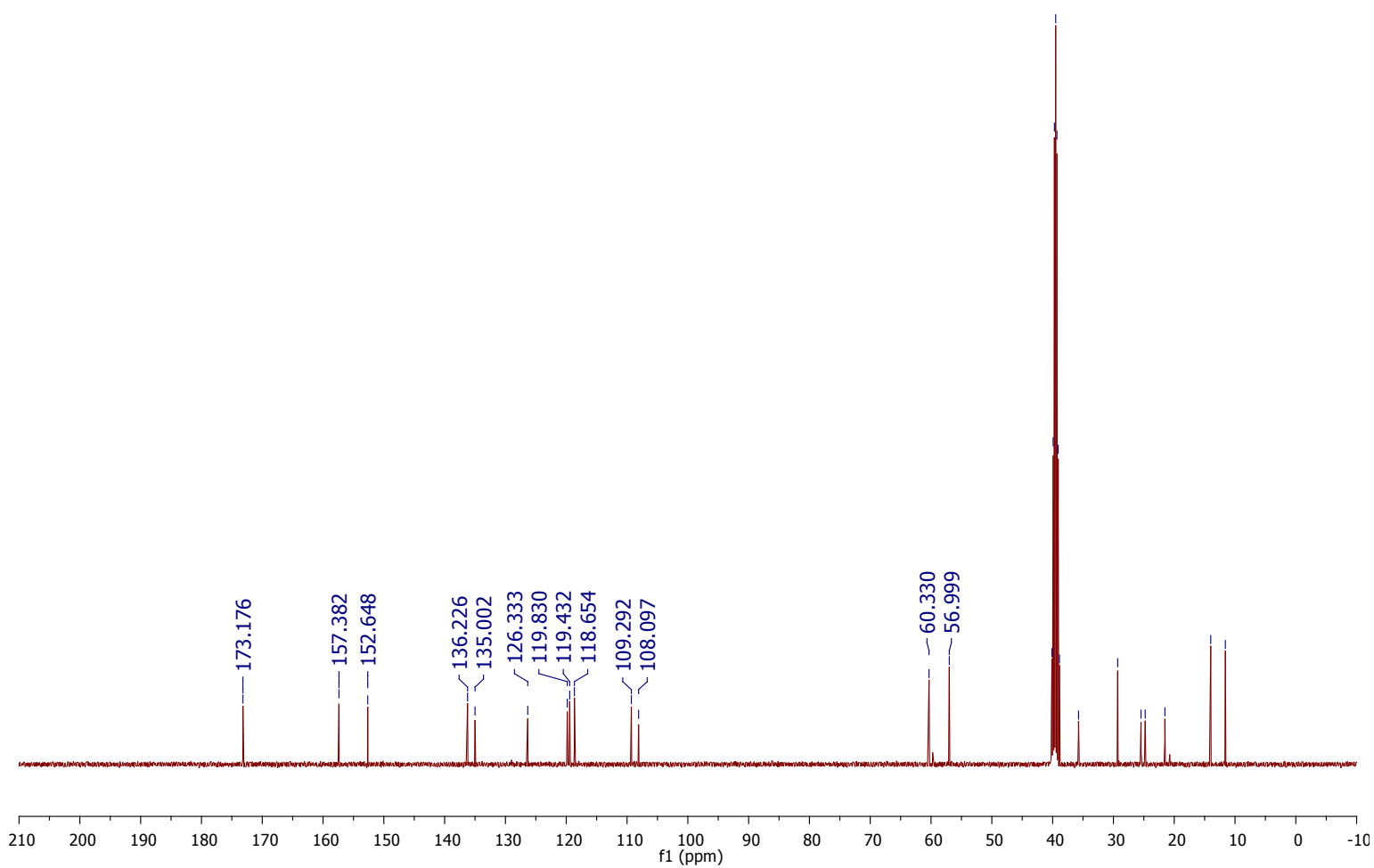


${ }^{1} \mathrm{H}$ NMR of 4e (400 MHz, DMSO- $\left.d_{6}\right)$
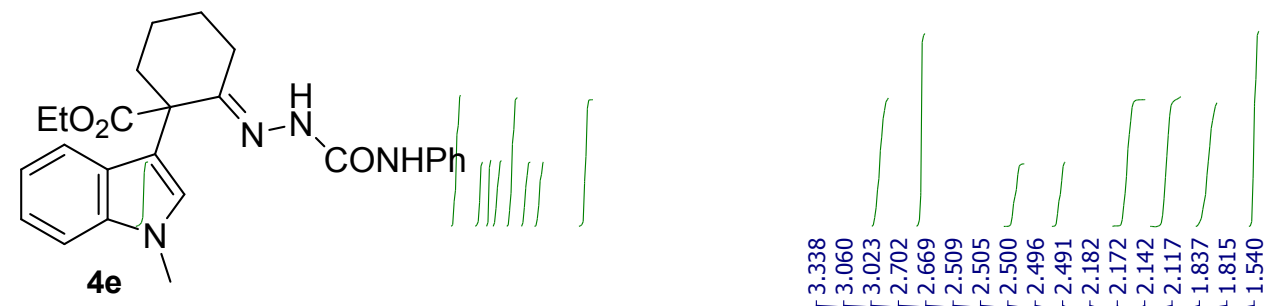

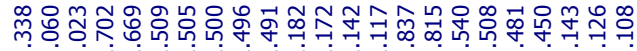

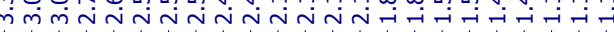

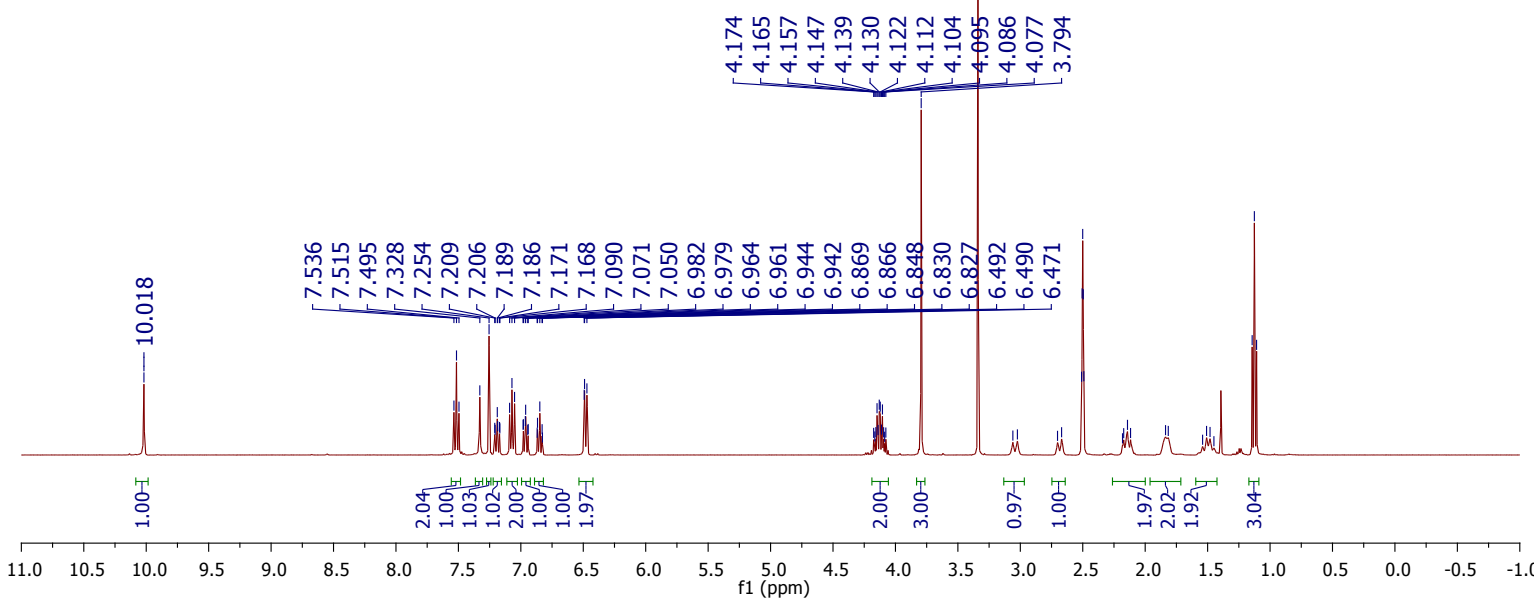

${ }^{13}$ C NMR of 4e (100 MHz, DMSO- $d_{6}$

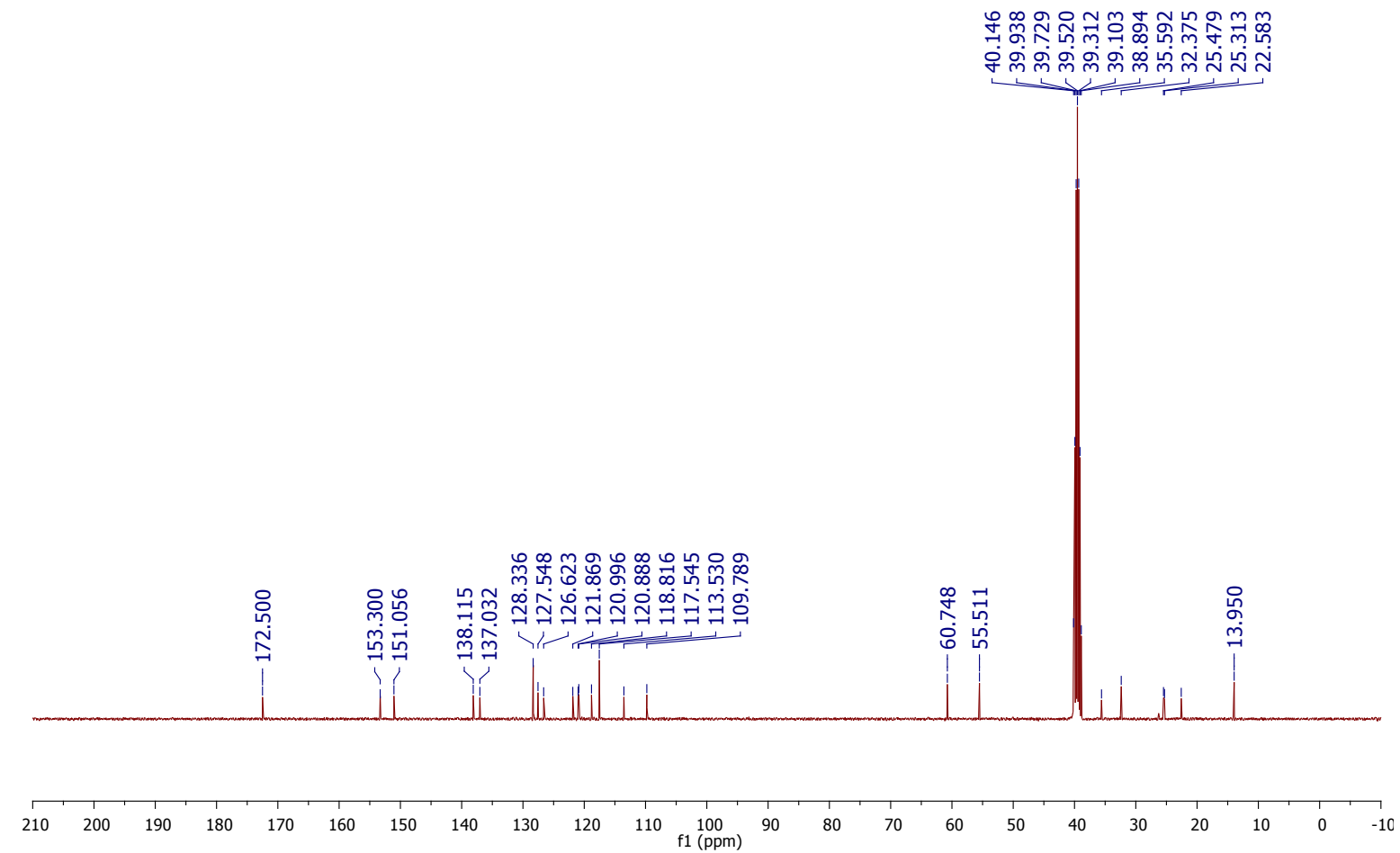


${ }^{1} \mathrm{H}$ NMR of $4 \mathrm{f}$ (400 MHz, DMSO- $d_{6}$

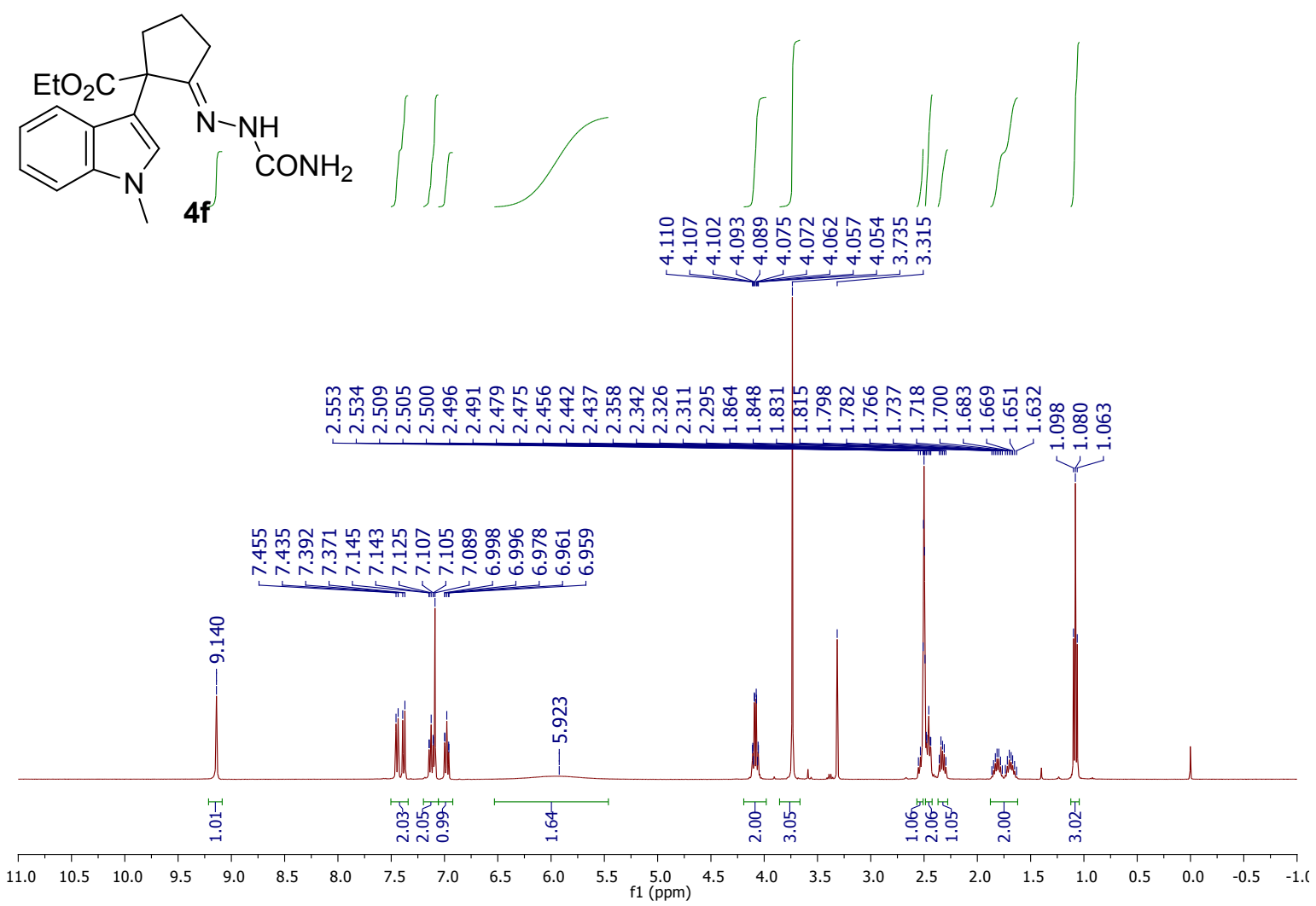

${ }^{13} \mathrm{C}$ NMR of $4 \mathrm{f}\left(100 \mathrm{MHz}\right.$, DMSO- $d_{6}$

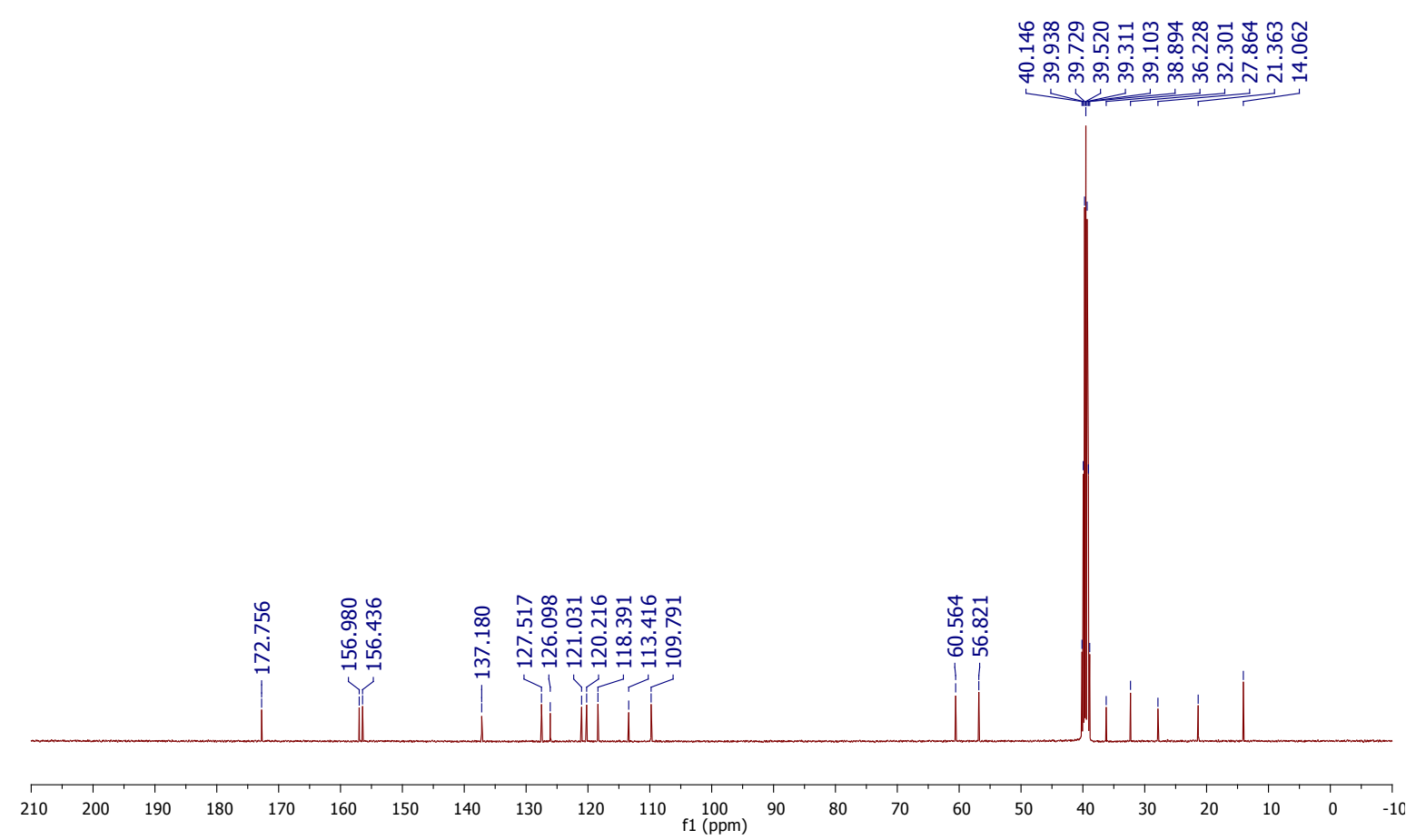


${ }^{1} \mathrm{H}$ NMR of 6a (400 MHz, DMSO- $d_{6}$<smiles>CC(=O)C1=C(C)NC2(C)N(Cc3ccccc3)c3ccccc3[C@]12C</smiles>
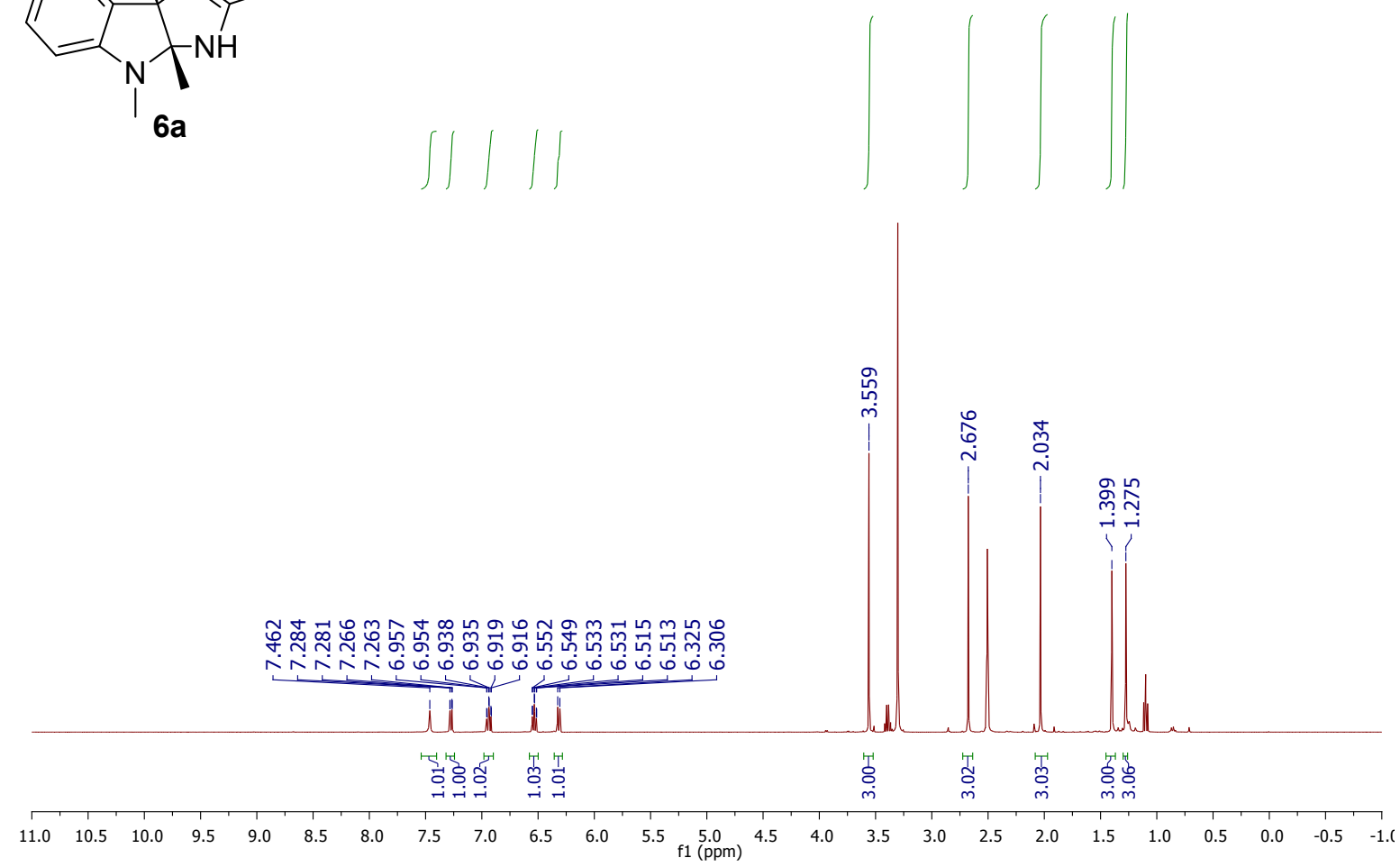

${ }^{13} \mathrm{C}$ NMR of $6 \mathrm{a}\left(100 \mathrm{MHz}\right.$, DMSO- $d_{6}$

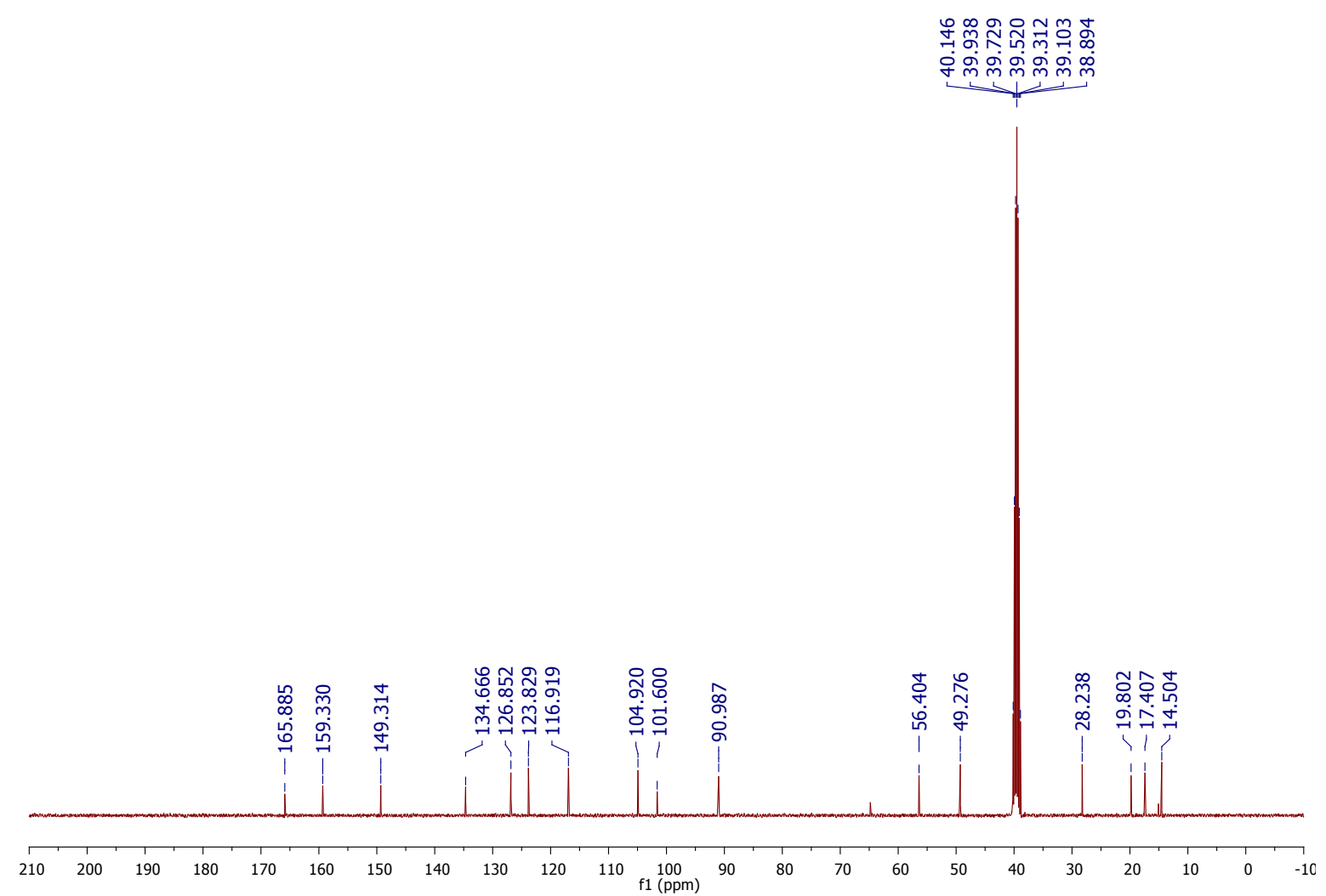


HMQC NMR of 6a (400 MHz, DMSO- $\left.d_{6}\right)$

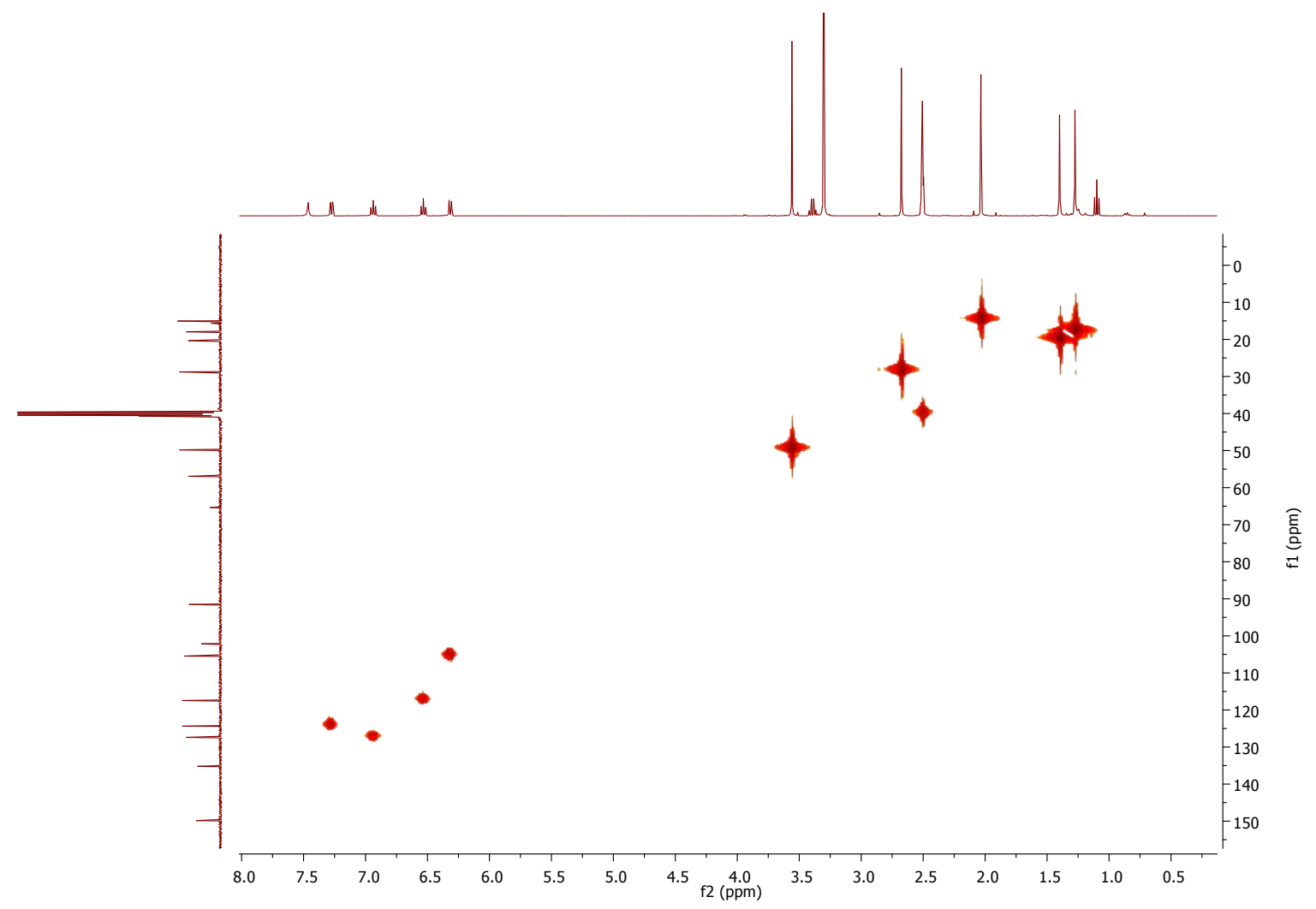

HMBC NMR of 6a (400 MHz, DMSO- $\left.d_{6}\right)$

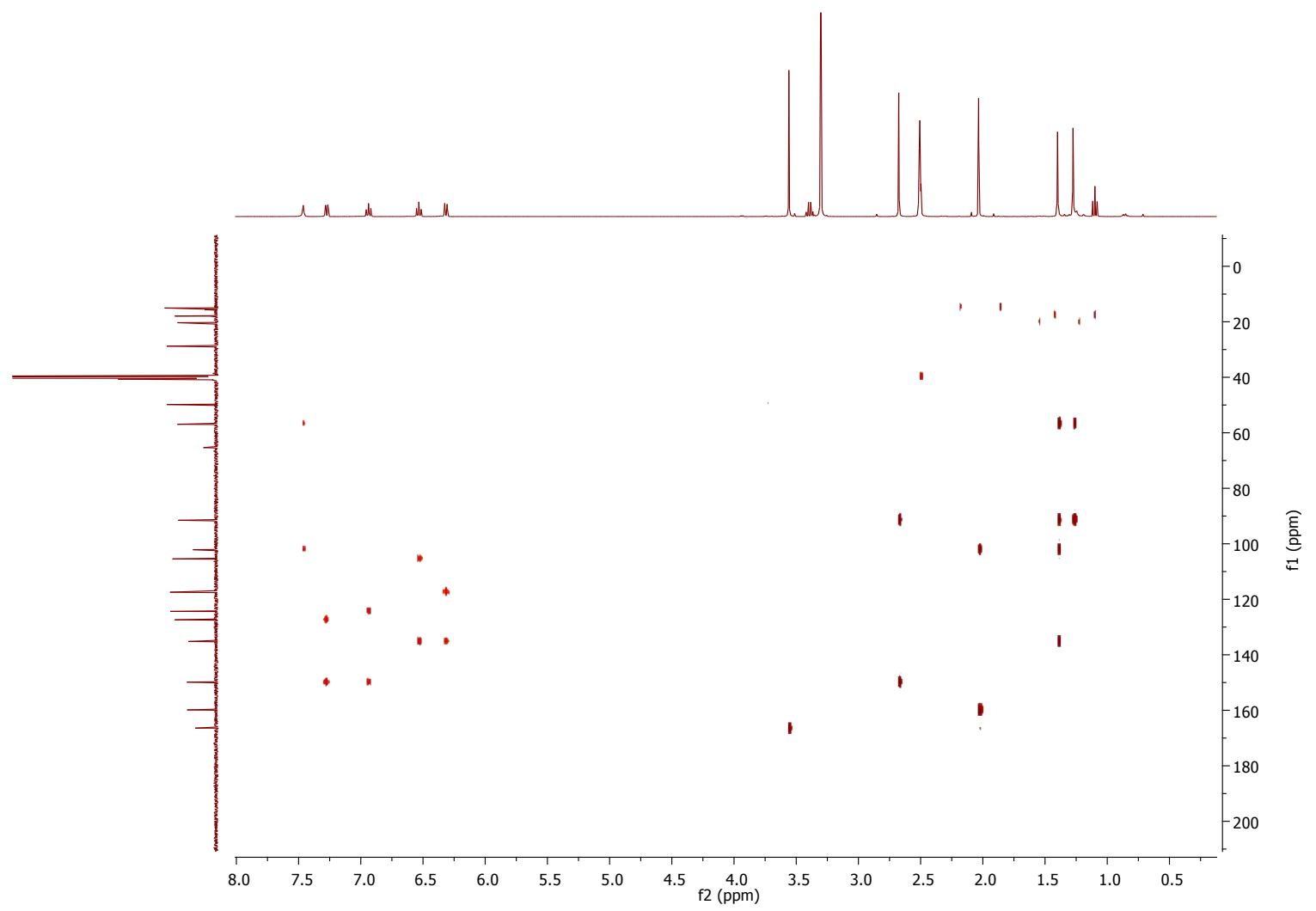

